US Army Corps

of Engineers ${ }_{\circledast}$

Engineer Research and

Development Center

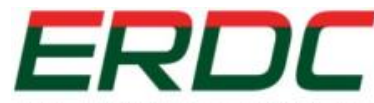

INNOVATIVE SOLUTIONS for a safer, better world

\title{
Lipophilic Super-Absorbent Swelling Gels as Cleaners for Use on Weapons Systems and Platforms
}

Veera M. Boddu, Sophie Minori Uchimiya, Masahiko Ohta,

February 2014

Kazuki Sada, Christopher Myers, Wayne Ziegler, and

Thomas Torres

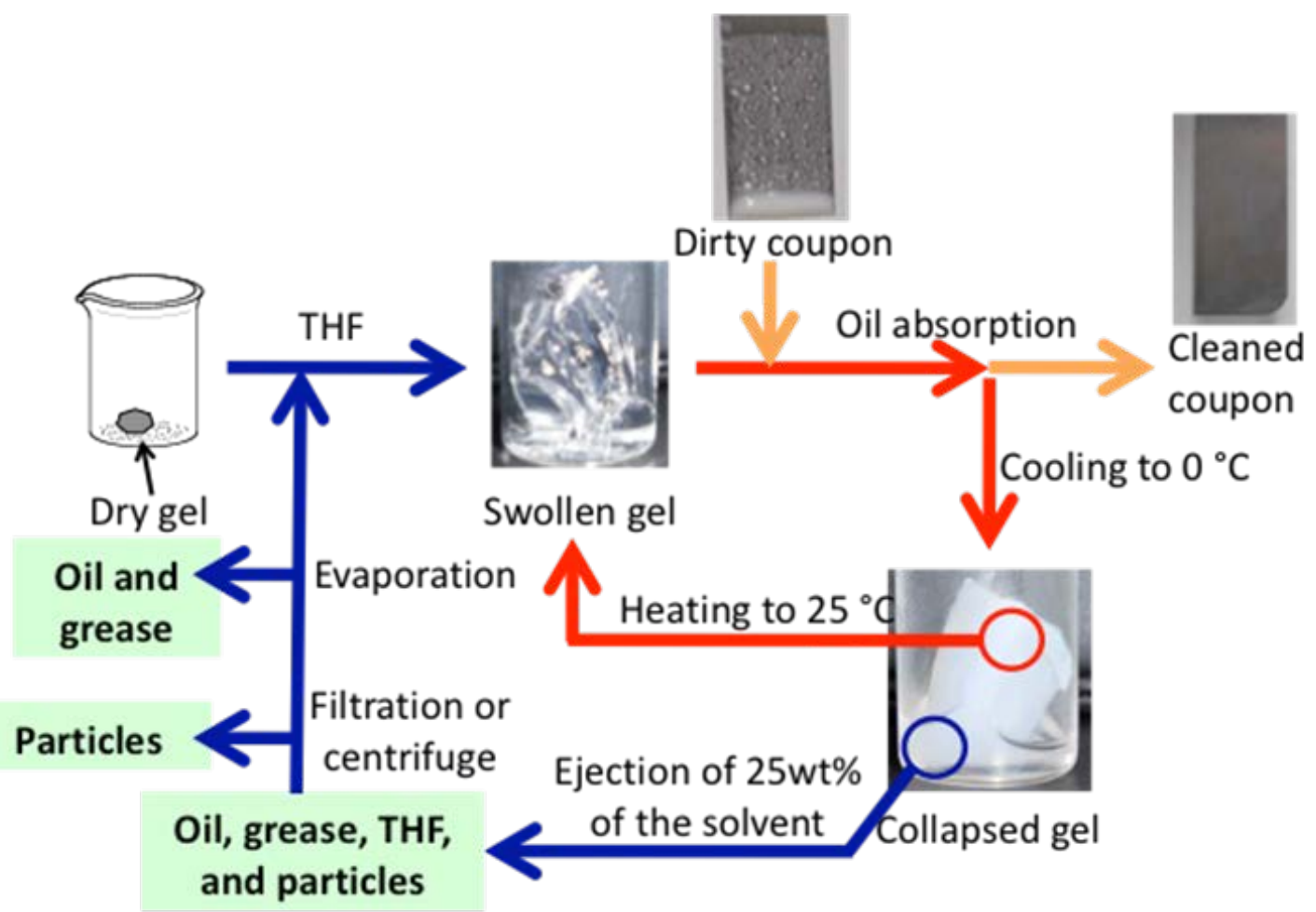


The US Army Engineer Research and Development Center (ERDC) solves the nation's toughest engineering and environmental challenges. ERDC develops innovative solutions in civil and military engineering, geospatial sciences, water resources, and environmental sciences for the Army, the Department of Defense, civilian agencies, and our nation's public good. Find out more at www.erdc.usace.army.mil.

To search for other technical reports published by ERDC, visit the ERDC online library at http://acwc.sdp.sirsi.net/client/default. 


\section{Lipophilic Super-Absorbent Swelling Gels as Cleaners for Use on Weapons Systems and Platforms}

Veera M. Boddu and Christopher Myers

Construction Engineering Research Laboratory (CERL)

U.S. Army Engineer Research and Development Center

2902 Newmark Dr.

Champaign, IL 61822-1076

Sophie Minori Uchimiya

Agricultural Research Service

U.S. Department of Agriculture

New Orleans, LA 70124

Masahiko Ohta and Kazuki Sada

Department of Chemistry, Hokkaido University

Sapporo, Hokkaido 060-0810, Japan

Wayne Ziegler

U.S. Army Research Laboratory

Aberdeen Proving Grounds, MD 21005-5069

Thomas Torres

U.S. Naval Facilities Engineering Service Center Port Hueneme, CA 93043

Final Report

Approved for public release; distribution is unlimited.

Prepared for Headquarters, U.S. Army Corps of Engineers

Washington, DC 20314-1000 


\section{Abstract}

Increasingly stringent environmental regulations on volatile organic compounds (VOCs) and hazardous air pollutants (HAPs) demand the development of disruptive technologies for cleaning weapons systems and platforms. Currently employed techniques suffer from safety and efficiency shortcomings. This work proposed to use novel lipophilic super-absorbent swelling gels as a disruptive solid state cleaning technology to overcome limitations of currently employed cleaning techniques. Results were compared with a commercially available alkylstyrene copolymer (imbiber beads). The cleaning ability of the gels was compared with the standard solvent cleaner trichloroethylene (TCE) following American Society for Testing and Materials (ASTM) G122-96(2008) test methods and MILPRF-680B procedure with MIL-PRF-10924 test grease. Polymer gel cleaners exhibited similar extent and rate of cleaning as the TCE. The work concludes that the recyclable superabsorbent polymer cleaners developed in this research will allow drastic reduction in the use of VOC containing solvents and HAP release.

DISCLAIMER: The contents of this report are not to be used for advertising, publication, or promotional purposes. Citation of trade names does not constitute an official endorsement or approval of the use of such commercial products. All product names and trademarks cited are the property of their respective owners. The findings of this report are not to be construed as an official Department of the Army position unless so designated by other authorized documents. 


\section{Contents}

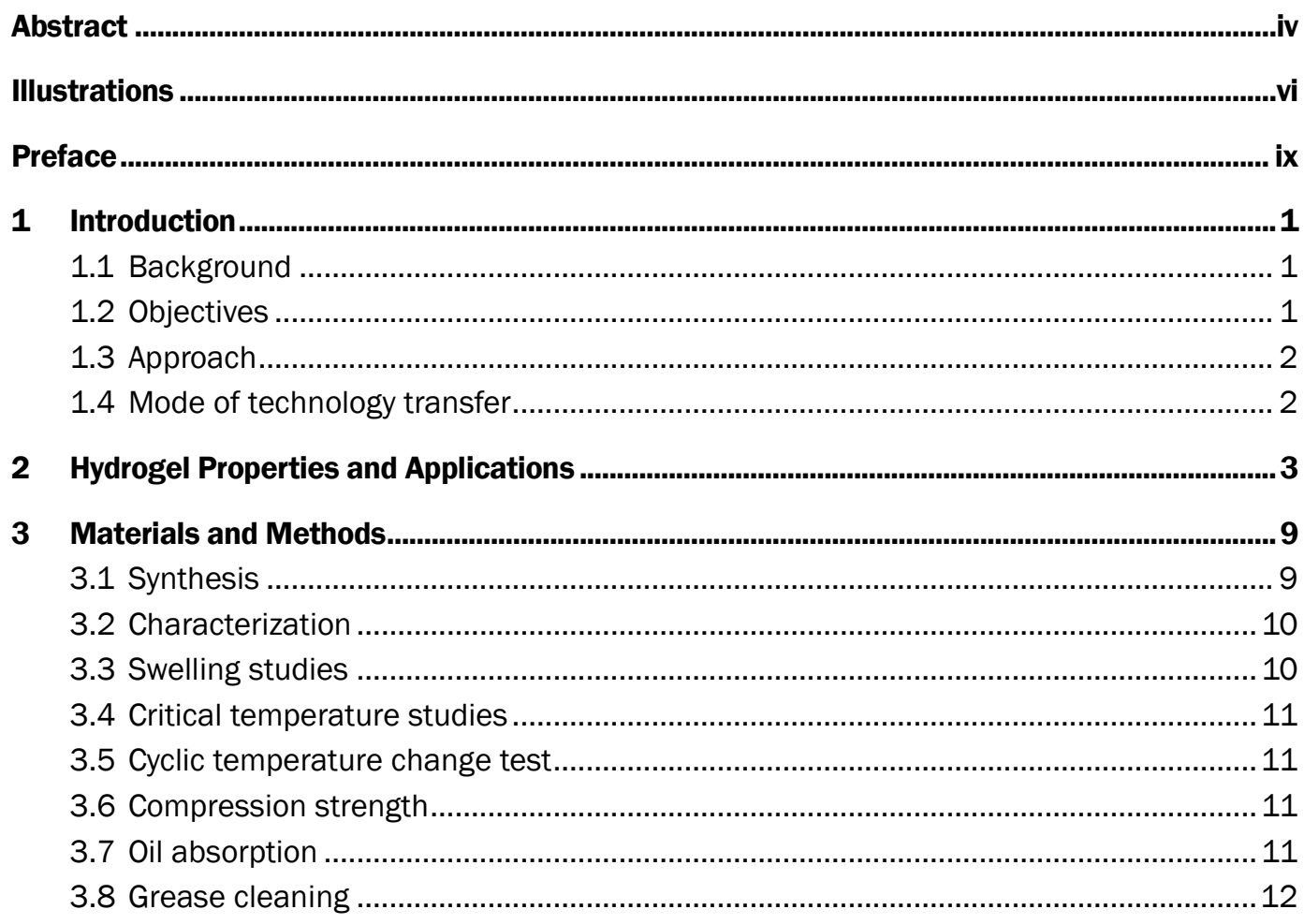

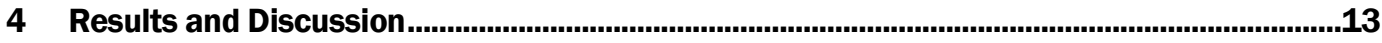

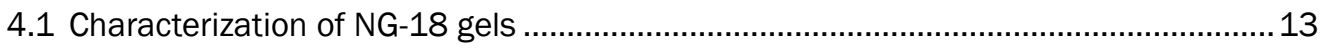

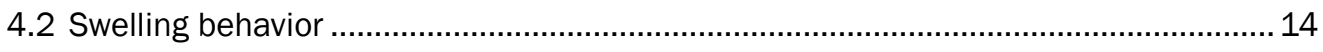

4.3 Critical solution temperature ............................................................................. 18

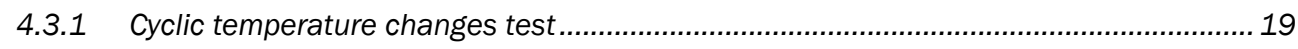

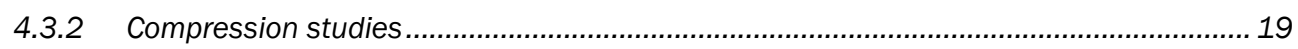

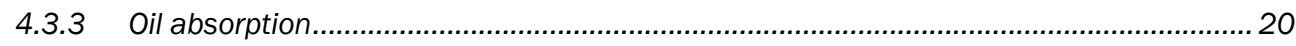

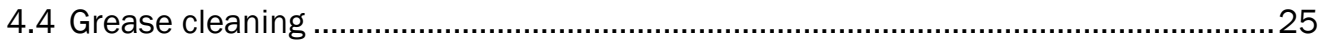

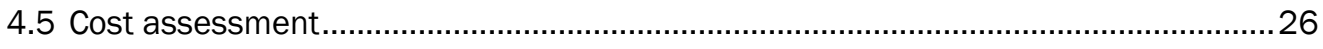

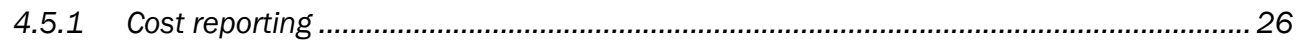

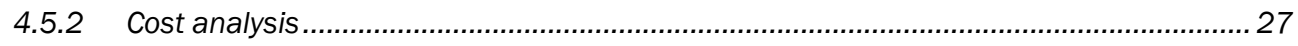

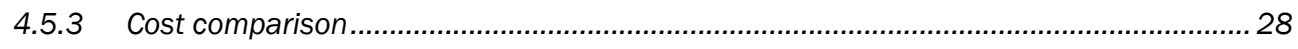

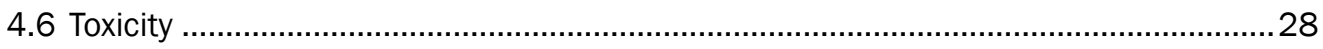

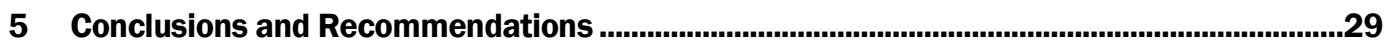

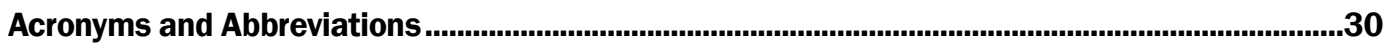

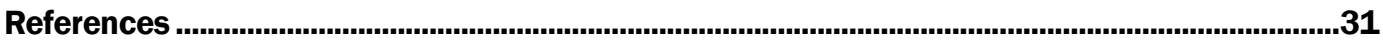

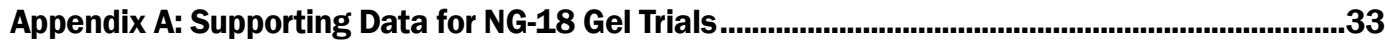

Appendix B: Material Safety Data Sheets Imbiber beads, TCE, and THF ......................................47

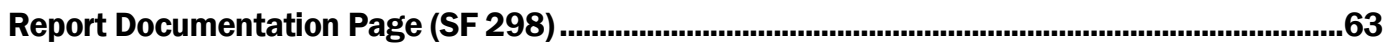




\section{lllustrations}

\section{Figures}

1 Dry (a) lipophilic polyelectrolyte gel (EG-18) swollen (b) in tetrahydrofuran (THF) $(\varepsilon=7.6)$. Dry (c) neutral analogue (NG-18) swollen (d) in THF. Illustrations are in scale with one another. (Illustration adapted from Reference 3 )

2 Preparation of candidate lipophilic tetraalkylammonium tetraphenylborate polyelectrolyte gel (EG-18) and its neutral analogue (NG-18) (Illustration adapted from Reference 3)

3 Swelling degree (Q) of lipophilic polyelectrolyte gels (EGn where $n$ represents alkyl chain length of polyacrylate polymer backbone, as shown in Fig. 2) and neutral analogues (NGn) in organic solvents (in increasing order of polarity from left to right). (Illustration adapted from Reference 4) ........................................................... 7

4 FTIR spectra of stearylacrylate .13

5 Compression strength of swollen NG-18-1\%, THF has a breaking point around

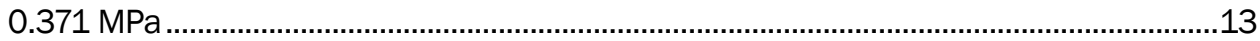

6 Swelling degree (Q) of NG-18-1\%, $-0.5 \%$ and imbiber beads in various solvents ..............15

7 Temperature dependence of swelling degree (Q) of NG-18-1\% in various solvents...........16

8 Comparison of heated record of NG-18 gels in the cooling process....................................17

9 Swelling degree changes of NG-18 with time in THF .........................................................18

10 (a) Temperature dependence of transmittance at $700 \mathrm{~nm}$ of NG-18-1\% gel swelled in THF. (b) Photograph of transparent state at $25^{\circ} \mathrm{C}$. (c) Photograph of opaque state at $\mathrm{O}^{\circ} \mathrm{C}$

11 (a) Results of temperature cycle $\left(25^{\circ} \mathrm{C} \rightarrow 0^{\circ} \mathrm{C}\right)$ test: changes of (circle) transmittance at $700 \mathrm{~nm}$, (triangle) swelling degree in THF. (b) Photograph of transparent state at 9th step. (c) Photograph of opaque state at 10th step...

12 Compression strength of swollen (a) NG-18-1\% and (b) NG-18-0.5\% in toluene................20

13 Oil absorption properties of NG-18 gels..........................................................................21

14 Metal surface cleaning properties of NG-18 gels ..............................................................21

15 Swelling degree of NG-18-x\% in various solvents after (a) 24 h, (b) 48 h, and (c) $72 \mathrm{~h}$

16 Oil recycling system with swollen NG-18-1\% gel in THF

17 Photographs of metal coupons (a) before and (b) after immersing the gel: from left side, cycle1-5. (c) Absorbed amount in each cycle

18 Photographs of (a) collected solution, (b) filtrated residual particulates, and (c) residual oil after evaporation. (d) Ratio of collected solution amount for the weight of gel on each cycle

19 Photographs of metal coupons (a)-(c) soaked in SAE-30 oil, (d)-(f) soaked in the mixture of SAE-30 oil and alumina powder, (g)-(i) immersed in NG-18-1\% gel, and (j) immersed in NG-18-0.5\% gel (circles indicate the alumina remaining area).....

20 Photographs of soiled metal parts cleaning test (a) before and (b) after cleaning............25

21 Grease absorption properties of NG-18 gels.....................................................................25

22 Cleaning power of NG-18 gels ............................................................................................26 
A-1 Metal coupons immersed in gels: (left) NG-18-1\%, (center) NG-18-0.5\%, and

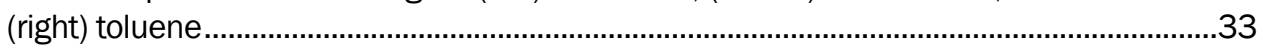

A-2 A photograph of the containers of (left) BioAct -MSO and (right) SAE-30 oil ......................36

A-3 Chemical structures of BioAct-MSO components: (a) 1-Methyl-4-(1methylethynyl)-cyclohexene (d,I-limonene), (b) sodium dioctylsulfosuccinate, (c) Poly(oxy- 1,2-ethanediyl), alpha-(4-nonylphenol)-omega-hydroxy ........................................36

A-4 Sol-gel transition of BioAct-MSO with increasing the water content.....................................37

A-5 Photographs of coupons soaking in (left) BioAct-MSO gel, (center) BioAct-MSO

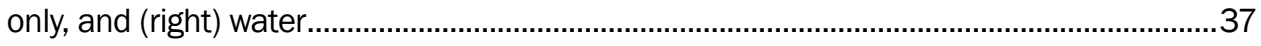

A-6 Photographs of metal coupon (a) coated with oil (b)-(d) after immersion: (b)

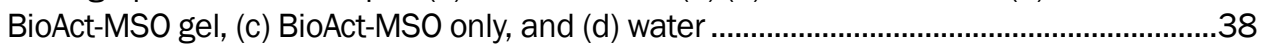

A-7 Photographs of metal coupons (a) coated with oil and alumina powder, (b)-(d) after immersion: (b) BioAct-MSO gel, (c) BioAct-MSO, and (d) water ...................................38

A-8 Cleaning properties of BIOACT-MSO ……….....................................................................38

A-9 Oil absorption properties of BioAct MSO compared to NG-18, water and THF....................39

A-10 Oil and alumina absorption properties of BioAct MSO and NG-18 gels compared

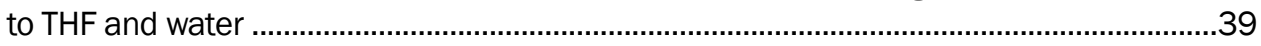

A-11 Cleaning Power of BioAct MSO and NG-18 gels compared to trichloroethylene ..................40

A-12 Grease absorption properties of BioAct MSO and NG-18 gels compared to trichloroethylene.

A-13 (a)-(c) Photographs of BIOACT-MSO+oil: from left side, 2, 4, 6, 8, 10, 15, 20, and $40 \mathrm{vol} \%$ of oil was contained. (a) before heated, (b) cooled to r.t. after heated to $60{ }^{\circ} \mathrm{C}$, and (c) separated gel phase after heated. (d) The changes of the amount of liquid phase after heated.

A-14 IR spectra of BioAct MSO, SAE-30 oil, and their mixtures. 1400-1500 cm-1 region was magnified to investigate the ratio of oil content in liquid and gel

A-15 The changes of $1455 \mathrm{~cm}-1$ with increment of oil content when the spectra were normalized at $1436 \mathrm{~cm}-1$ : (a) liquid phase, (b) gel phase.....................................................42

A-16 The intensities of 1455 and $1426 \mathrm{~cm}-1$ in each spectrum were indicated as a-f..............43

A-17 The oil content of each phase calculated from intensities of IR spectra: (left) liquid phase, (right) gel phase

A-18 (a) Photographs of the filtrate by using different pore size filter: from left side, $0.1,0.2$, and 1-5 $\mu \mathrm{m}$. (b) A photograph of the filtration apparatus. (c) IR spectra of each components, residue, and filtrate.

In 44

A-19 Metal coupon (a) coated with oil, and after immersion in: (b) BioAct MSO gel,

(c) BioAct MSO, and (d) water

A-20 Coupons soaking in (left) BioAct MSO gel, (center) BioAct MSO only, and (right) water.

A-21 Metal coupons (a) coated with oil and alumina powder, and after immersion in:

(b) BioAct MSO gel, (c) BioAct MSO, and (d) water.

A-22 Uncontaminated metal coupon (a), contaminated metal coupon (b), and coupons cleaned with: TCE (c), and BioAct MSO with $17 \%$ water (d) and $21 \%$ water (e) 


\section{Tables}

1 Lagergren first and second rate constants ( $k 1$ and k2) for swelling of the NG-18

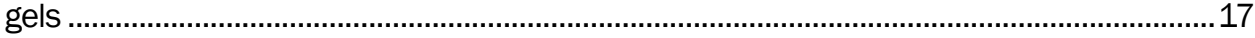

2 Chemical cost and amounts needed to make one kilogram of dry NG-18 gel...................27

3 Chemical amounts of NG-18 and trichloroethylene needed to fill a $3400 \mathrm{~L}$

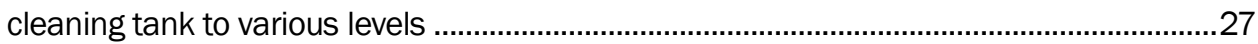

4 Cost per batch of NG-18 gels compared to trichloroethylene when cleaning tank is filled to various levels

5 Cost over time of NG-18 gels compared to trichloroethylene when cleaning tank is filled to various levels assuming no depreciation and one use per day .....

A-1 Metal coupon before (b), and after (a) being contaminated with MIL-PRF-10924 grease. Metal coupons cleaned with NG-18 0.5\% (c)-(d) for 13 minutes and 32 seconds, TCE for 5 minutes 12 seconds (e), and NG-18 1\% for 12 minutes 58 seconds (f) 34

A-2 Experimental grease absorption data of NG-18 gels ....................................................34

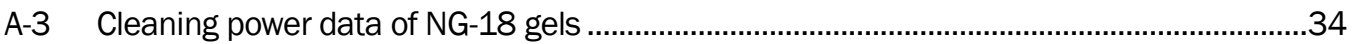

A-4 Swelling data of NG-18-x\% gel in various solvents for $24 \mathrm{~h}, 48 \mathrm{~h}$, and $72 \mathrm{~h}$......................35

A-5 The list of filter used in this experiment .........................................................................4

A-6 Experimental grease absorption data of NG-18 gels .......................................................

A-7 Cleaning power data of BioAct MSO gels ............................................................................45 


\section{Preface}

This study was conducted for the Strategic Environmental Research and Development Program (SERDP) under Project No. WP-1761, "Weapons Storage and Platforms."

The work was performed by the Environmental Processes (CN-E) Branch of the Installations Division (CN), Construction Engineering Research Laboratory (CERL). The CERL Principal Investigator was Dr. Veera Mallu Boddu. At the time of publication, Deborah Curtin was Chief, CN-E and Michelle Hanson was Chief, CN. The associated Technical Director was Alan Anderson. The Deputy Director of CERL is Dr. Kirankumar V. Topudurti, and the Director is Dr. Ilker R. Adiguzel.

The Commander and Executive Director of ERDC is COLJ effrey R. Eckstein, and the Director of ERDC is Dr. J effery P. Holland. 


\section{Introduction}

\subsection{Background}

Polyelectrolyte gels are ionic polymer networks composed of charged polymer chains and freely mobile counter-ions. Polyelectrolyte superabsorbent wet-swelling hydrogels are known to undergo a dramatic but reversible volume change by absorbing large quantities of water. Polyelectrolyte hydrogels swell in water because of:

1. Osmotic pressure induced by freely mobile counter-ions within the polyelectrolyte

2. Increased entropy arising from the solvation of polymer ions and counterions

3. Electrostatic repulsion between the oppositely charged ions within the polyelectrolytegel

4. Stretching of polymer chains between crosslinks caused by the increase in entropy associated with mixing polymer with solvent (Ohmine and Tanaka 1982).

These properties give super absorbent polymer gel systems a pronounced capability for removing oil, grease, and particulates from metal and plastic surfaces This work was undertaken to develop a new cleaning technology based on lipophilic super-absorbent swelling gels that is VOC-exempt, HAP-free, non-toxic, non-corrosive, non-ozone depleting, and recyclable.

\subsection{Objectives}

The overall objective of this research was to develop an environmentally benign disruptive technology for cleaning metal and non-metal surfaces, specifically by developing materials formulated to maximize the cleaning efficiency of lipophilic gels in the following steps:

1. Two potential gels were identified, a lipophilic polyelectrolyte(EG-18) gel and a neutral (NG-18) polymer gel for surfacecleaning applications. This initial phase of the study evaluates these gels following American Society for Testing and Materials (ASTM) G122-96(2008) and MIL-PRF-680B protocols.

2. Representative contaminants of oil, grease and particulate materials were selected.

3. Testing cleaning ability of candidates EG-18 and NG-18 gels 
4. Designing lipophilic gels with improved cleaning ability by appropriate Selection of lipophilic polymer backbone, weakly coordinating anions, and enhancement of mechanical strength.

\subsection{Approach}

The objectives of this work were achieved in the following steps:

1. The ability of two super absorbent polymer gel systems for removing oil, grease, and particulates from metal and plastic surfaces were evaluated.

2. Once the surface cleaning ability of these gel systems was demonstrated, subsequent research focused on improving the gel performance by design and synthesis of additional polymer gel systems.

3. Further research addressed the post-cleaning gel removal method, the use of non-fluorinated compounds in gel synthesis, and an evaluation of toxicity and environmental fate-and-effects of the gels.

4. The study used intricate designing of lipophilic super-absorbent swelling gels through careful selection of polymer backbone and ionic components, and the cross linking density to improve the cleaning ability of thelipophilic swelling gels.

\subsection{Mode of technology transfer}

The proposed scope of the full project will elucidate the chemical and physical mechanisms in the removal of oil, grease, and particulate contaminants from metal and non-metal surfaces by lipophilic super-absorbent swelling gels. Research outcomes will facilitate the development of environmentally compliant, economically feasible cleaners for a wide range of DoD applications, providing a promising alternative to traditional vapor degreasing, solvent, aqueous, or blast cleaning processes. Environmentally benign VOC-exempt, and HAP-free surface cleaning technology, will support ongoing DoD programs such as the Sustainable Painting Operations for Total Army (SPOTA). Technology developed in this research will result in dramatic overall reductions of VOCs and HAPs emissions from DoD surface cleaning operations. It is anticipated that the results of this work will be used to address associated issues, including:

1. The method for removing the gels after swelling

2. The use of non-fluorinated compounds in gel synthesis

3. An evaluation of toxicity and environmental fate-and-effects of the gels

4. Elimination of fluorinated compounds in the gel synthesis. 


\section{Hydrogel Properties and Applications}

Polyelectrolyte hydrogels have found a wide range of applications in diapers, inks and display devices, separation media, and clean up of aqueous spills. Polyelectrolyte hydrogels are particularly useful for a wide range of environmental applications because expansion and contraction of the gels can be engineered to be triggered by small changes in environmental parameters such as temperature, $\mathrm{pH}$, and ionic strength. However, until recently, reports on gels that will swell by absorbing large quantities of nonpolar organic solvents were nearly nonexistent. In nonpolar solvents, most polyelectrolyte gels collapse, because the oppositely charged ions within the gel form ion pairs that then aggregate, rather than becoming solvated.

In 2007, Ono et al. (2007) reported for the first time a novel class of lipophilic polyelectrolyte gels bearing positively charged repeating units (substituted tetraalkylammonium with long alkyl chains) and negatively charged counter-ions (substituted tetraphenylborate; TFPB-) that swell dramatically but reversibly by absorbing organic solvents having various polarities ( $\varepsilon=1.9-46$; the lower the dielectric constant $(\varepsilon)$, the less polar the solvent). Figure 1(a)-(b) shows how superior swelling ability in nonpolar solvents is enabled by making both the polymer chains and the counter-ions lipophilic, preventing counter-ions from forming ion pairs, thereby enabling the solvation of ionic gel components in solvents. Lipophilic polyelectrolyte gel presented in Figure 1 (a)-(b) is hereby termed EG-18 and will serve as a candidate cleaner in this proposal.

Figure 1(c)-(d) illustrates swelling behavior of NG-18, a neutral analogue of EG-18 that does not contain the ionic tetraalkylammonium tetraphenylborate unit. As shown in Figure 1, neutral gel NG-18 swells to a much lesser extent than the ionic EG-18 gel. Neutral polymer gels swell in organic solvents because of the stretching of polymer chains between crosslinks caused by the increase in entropy associated with mixing polymer with solvent. Additional swelling mechanisms of polyelectrolyte gels such as the solvation of ionic groups do not exist in neutral gels. Therefore, neutral gels may be of limited use as cleaners compared to polyelectrolyte gels, but can be used as a measure of swelling capacity arising from the compatibility of the polymer chains with solvents alone. Figure 2 presents pathways for preparing the lipophilic polyelectrolyte swelling gel (EG-18) 
whose swelling behavior in organic solvent was presented in Figure 1(top). Figure 2 shows how tetraalkylammonium tetraphenylborate polyelectrolyte gel (EG-18) can be prepared in three steps.

As illustrated in Figure 2, the ratio of ionic unit tetraalkylammonium tetraphenylborate (p), polymer backbone ODA (q), to cross linker EGDMA ( $r$ ) for EG-18 is kept at p:q: $r=5: 95: 1$ for EG-18 to maintain low content of ionic groups (Ono et al. 2008). Ionic group content must be kept low to avoid aggregation of ionic groups. Neutral analogue NG-18 can be prepared by simply excluding the ionic tetraalkylammonium tetraphenylborate unit ( $p$ ) for the feed ratio of $p: q: r=0: 100: 1$.

Figure 3 shows the impact of crosslinked polyacrylate polymer backbones on the swelling degree of ionic (EGn) and neutral (NGn) gels. Gels shown in Figure 3 possess polyacrylate backbones with alkyl chain lengths ranging from $\mathrm{n}=18\left(\mathrm{R}=\left(\mathrm{CH}_{2}\right)_{17} \mathrm{CH}_{3}\right), 16\left(\mathrm{R}=\left(\mathrm{CH}_{2}\right)_{15} \mathrm{CH}_{3}\right), 12\left(\mathrm{R}=\left(\mathrm{CH}_{2}\right)_{11} \mathrm{CH}_{3}\right)$, to $6\left(\left(\mathrm{R}=\left(\mathrm{CH}_{2}\right)_{5} \mathrm{CH}_{3}\right)\right.$; see ODA structures in Figure 2). Swelling degrees ( $\mathrm{Q}$ in wt/ wt) were quantitatively determined by soaking a selected gel in an appropriate solvent for a fixed time using the following equation:

$$
\mathrm{Q}=\frac{\mathrm{W}_{\mathrm{wet}}-\mathrm{W}_{\mathrm{dry}}}{\mathrm{W}_{\mathrm{dy}}}
$$

where $\mathrm{W}_{\mathrm{dry}}$ and $\mathrm{W}_{\text {wet }}$ are the weights of the dry and swollen gels, respectively.

Figure 3 shows that maximum absorbency of the polyelectrolyte gel (EGn) shifts toward solvents with lower polarity as alkyl chain length (i.e., lipophilicity) of polyacrylate backbone increases from $n=6,12,16$, to 18 . That is, EG-18 exhibits maximum swelling by absorbing large quantities of organic solvents with dielectric constants between 3 and 10 . On the other hand, EG6 shows maximum absorbency for much more polar solvents $(\varepsilon=16-32)$. 
Figure 1. Dry (a) lipophilic polyelectrolyte gel (EG-18) swollen (b) in tetrahydrofuran (THF) $(\varepsilon=7.6)$. Dry (c) neutral analogue (NG-18) swollen (d) in THF. Illustrations are in scale with one another. (Illustration adapted from Reference 3 ).
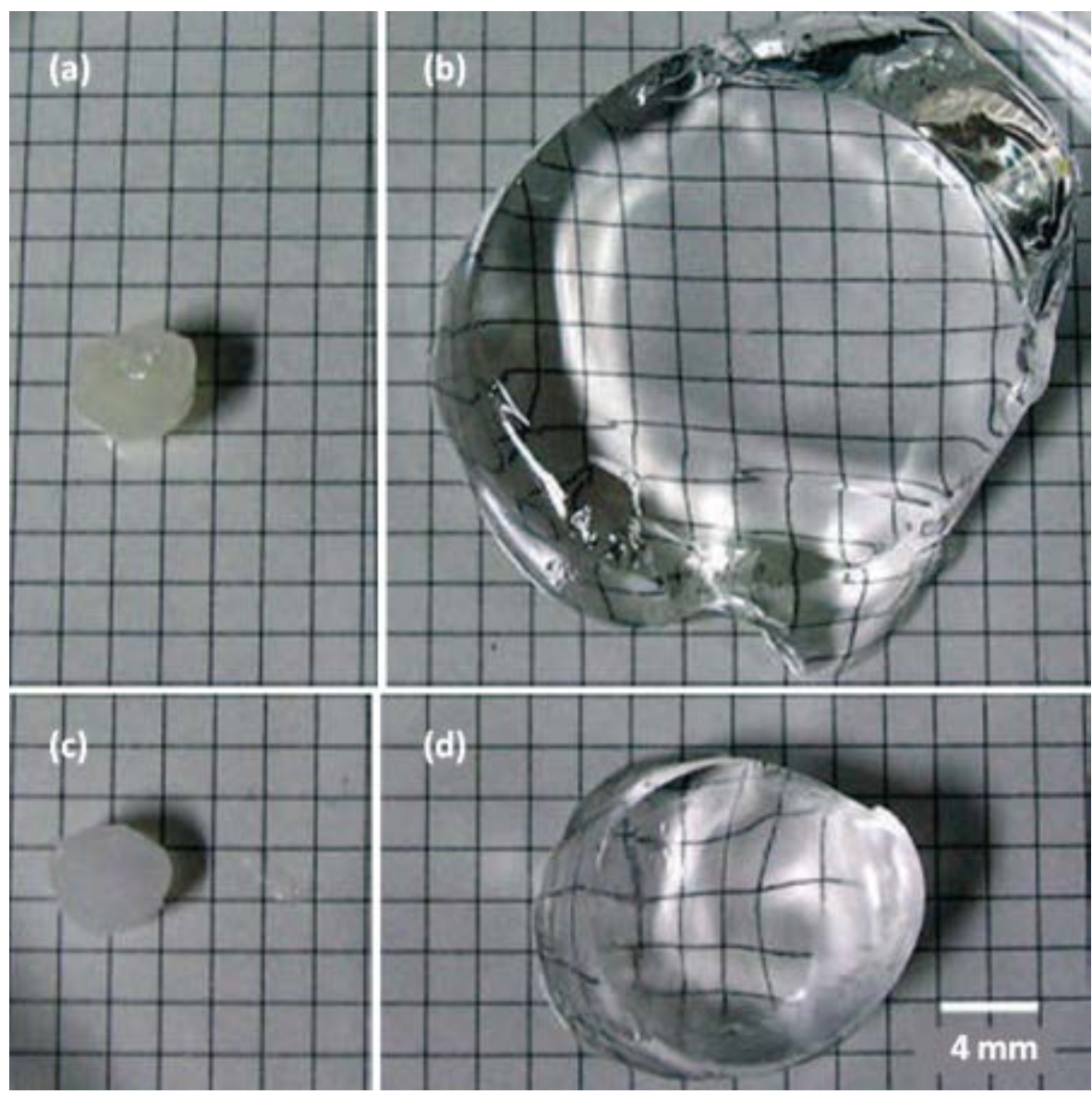
Figure 2. Preparation of candidate lipophilic tetraalkylammonium tetraphenylborate polyelectrolyte gel (EG-18) and its neutral analogue (NG-18) (Illustration adapted from Reference 3).

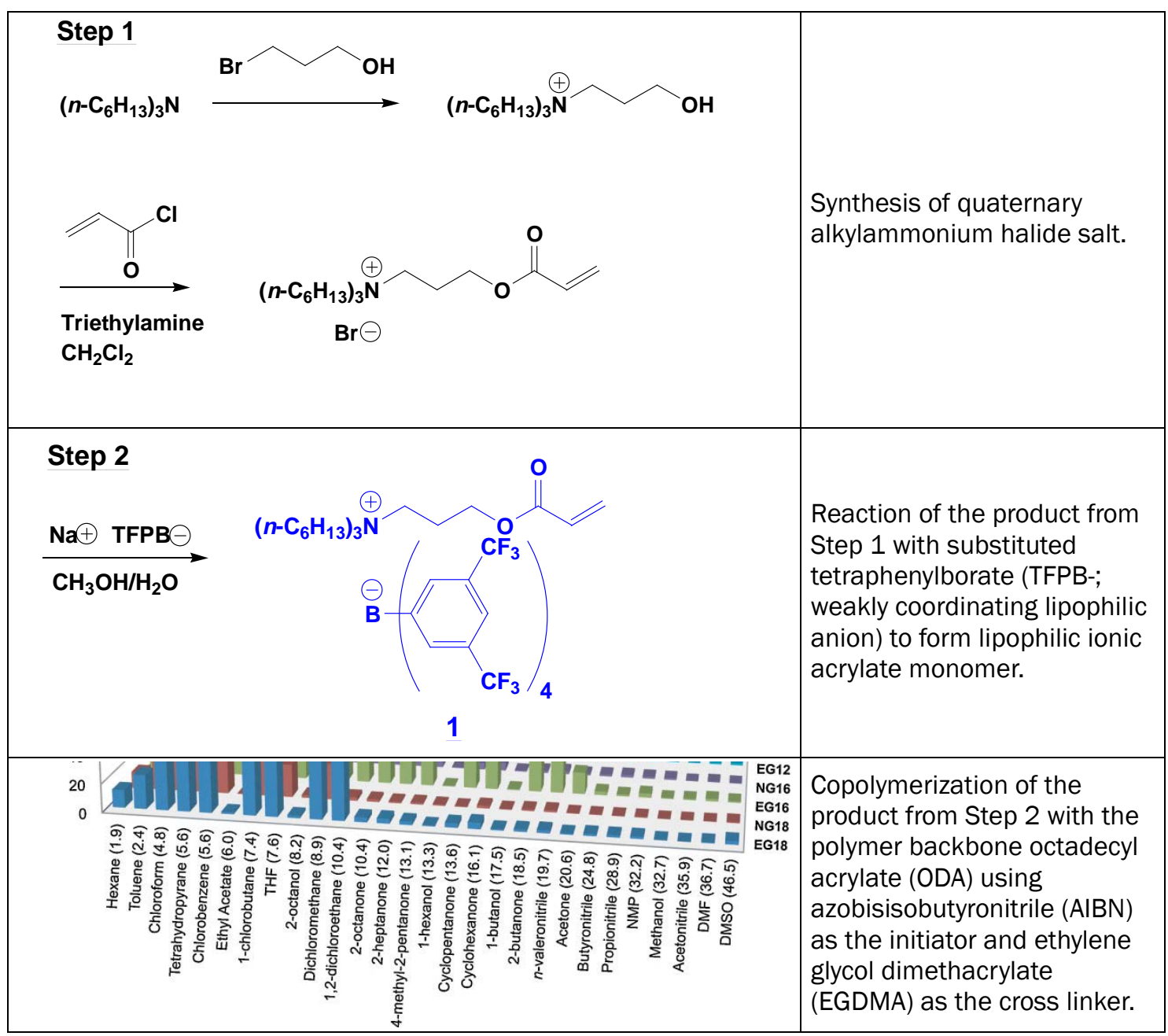


As shown in Figure 3, for a given alkyl chain length (n), ionic gel (EGn) swells to a much greater degree than the neutral analogue (NGn), as demonstrated visually for EG-18 and NG-18 in Figure 1. In solvents having dielectric constants below 3, comparable degrees of swelling are observed for ionic and neutral gels (Figure 3). In such nonpolar solvents, dissociation of ions within EGn is suppressed and ionic groups are tightly bound as ion pairs. As a result, the swelling of EGn results only from stretching of polymer chains between crosslinks caused by the increase in entropy associated with mixing polymer with solvent.

The following conclusions can be made from the review of recent and ongoing studies on polyelectrolyte and neutral lipophilic swelling gels provided above:

1. Swelling degree and absorbency of lipophilic polyelectrolyte gels are much greater than their neutral analogues.

2. Increased lipophilicity of both polymer backbones and ionic groups results in greater swelling capacity and absorbency in solvents having low dielectric constants.

Figure 3. Swelling degree (Q) of lipophilic polyelectrolyte gels (EGn where $n$ represents alkyl chain length of polyacrylate polymer backbone, as shown in Fig. 2) and neutral analogues (NGn) in organic solvents (in increasing order of polarity from left to right). (Illustration

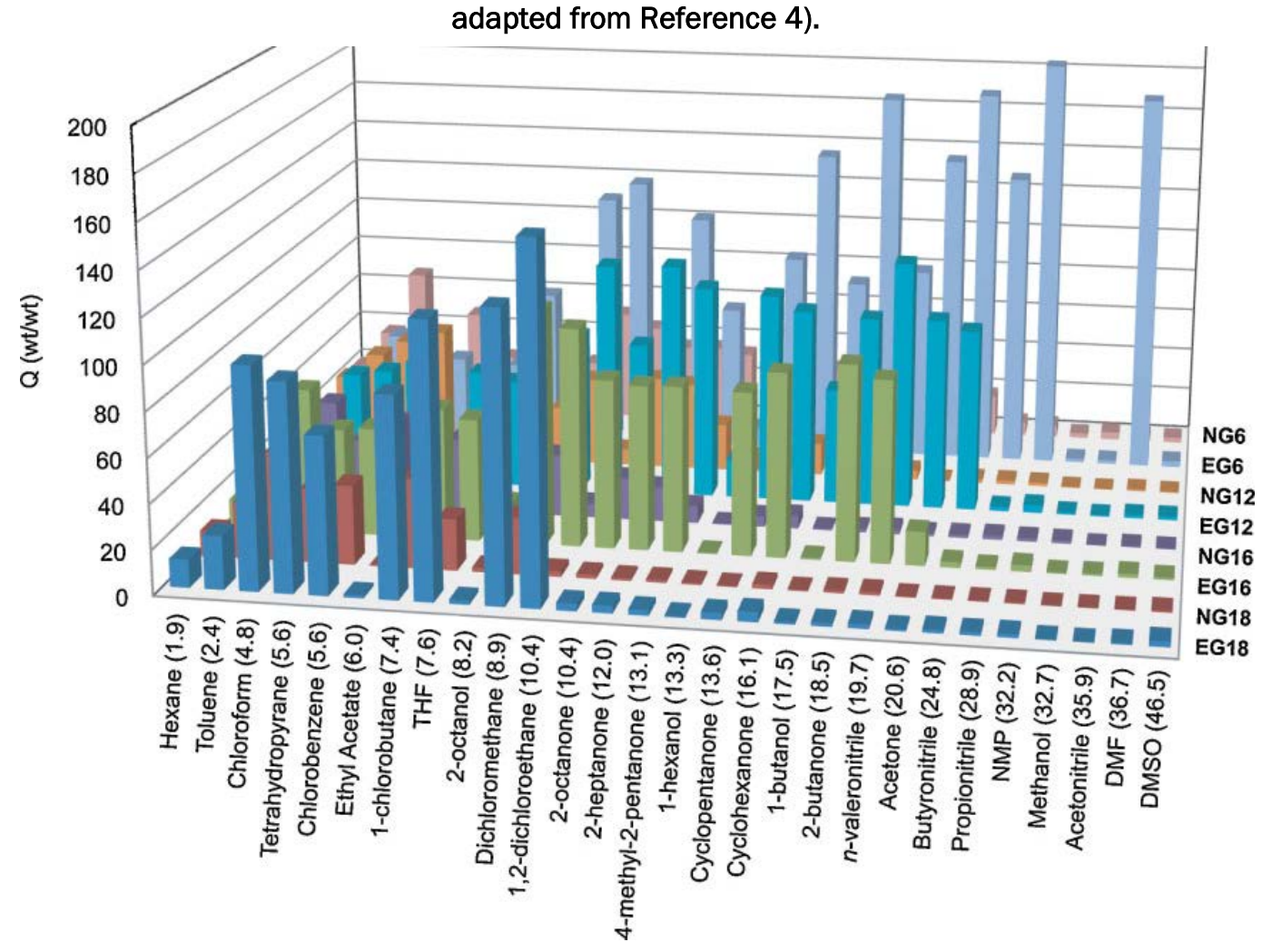


A promising candidate is the lipophilic super-absorbent gel that will swell by absorbing nonpolar organic solvents (e.g., hydrocarbon oils, VOCs) several hundred times their dry weight. This study aims to use intricate designing of lipophilic super-absorbent swelling gels through careful selection of polymer backbone and ionic components, and the cross linking density. Designed swelling gels will function as the cleaner of the metal and non-metal surfaces by: (1) absorption of oil and grease, and (2) removal of particulate contaminants by self-generated mechanical forces obtained from swelling in (1). After the cleaning operation, cleaning media can be safely collected and recycled or used in fuel blends.

Current limitations stated above call for a portable cleaning technology that will not pose environmental or health threats during or after the cleaning operations. The proposed cleaner is in solid form and is VOCexempt, HAP-free, non-toxic, non-corrosive, non-ozone depleting, recyclable, and self-generates the energy necessary for the cleaning function, thereby affording a new cost-effective, environmentally friendly cleaning technology. It is hypothesized that lipophilic super-absorbent swelling gels will, upon contacting oil and grease on the metal and non-metal surfaces, exert enough mechanical forces by swelling to remove particulate matters, oil, and grease on the material surfaces simultaneously.

The super-absorbent gels will exhibit low friction behaviors and therefore will not stick to or damage the surface of metal and nonmetal materials. Existing solvent and blast cleaning technologies pose environmental concerns both during and after the cleaning operation. During the cleaning operation, VOCs are produced from organic solvents and HAPs are produced from forced-air blast cleaning processes. After the cleaning operation, disposal of waste streams for solvent cleaning and cleanup of blasted contaminants for forced air cleaning have a potential to cause environmental problems.

In addition, techniques described here require on-site equipment such as the soaking bath and air compressor, and often necessitate operation in a confined, well-ventilated space. 


\section{Materials and Methods}

All chemical reagents were used as received. Stearylacrylate (SA), ethylene glycol dimethacrylate (EGDMA), benzene, azobisisobutylonitrile (AIBN), methanol, ethanol, diethylether, and carbon tetrachloride were purchased from Sigma-Aldrich (Milwaukee, WI). Tetrahydrofuran (THF), isopropanol, acetonitrile, and dichloromethane were obtained from Acros Organics (Morris Plains, NJ ). Dimethylsulfoxide (DMSO) and 1-octanol were supplied from Alfa Aesar (Ward Hill, MA). Chloroform and cyclohexane were distributed from VWR International (West Chester, PA). Acetone, methylisobutylketone (MIBK), toluene, and n-hexane were supplied from Fisher Scientific (Pittsburgh, PA). BioAct MSO was supplied by Petroferm Cleaning Products (Gurnee, IL). An Instron 5900 Electromechanical System was used for the compression of the gels. A J asco FT/IR-4100 Fourier Transform Infrared Spectrometer was used for infrared spectroscopy.

\subsection{Synthesis}

A typical protocol for the synthesis of NG-18 gel is as follows: $10.0 \mathrm{~g}$ (30.8 $\mathrm{mmol}$ ) of SA (monomer) and $61 \mathrm{mg}(0.31 \mathrm{mmol}$, the case of $\mathrm{x}=1)$ of EGDMA (crosslinker) as initiator were placed in a vial tube and dissolved in $2 \mathrm{~mL}$ of benzene by heating at $50^{\circ} \mathrm{C}$. Oxygen in the solution was excluded by bubbling nitrogen gas for $45 \mathrm{~min}$ then $101 \mathrm{mg}$ ( $0.62 \mathrm{mmol}$ ) of AIBN was added. The vial tube was sealed tightly and heated at $65^{\circ} \mathrm{C}$ for $24 \mathrm{~h}$ for polymerization. Gels with low crosslinking densities were prepared in a similar way by reducing the feed ratios of EGDMA. The synthesized gels were washed by swelling in hexane repeatedly, air-dried for 2 days, and dried in vacuum overnight. SA-co-EGDMA with two crosslinker ratios were prepared by radical copolymerization, which are represented as NG$18-\mathrm{x} \%(\mathrm{x}=1$ or 0.5 ; $\mathrm{x}$ denotes the mole ratio of crosslinker to $\mathrm{SA})$.

A typical protocol for the synthesis of EG-18 gel is as follows: $125 \mathrm{mg}(0.1$ $\mathrm{mmol})$ of TFPB and $617 \mathrm{mg}$ ( $1.9 \mathrm{mmol})$ of ODA, $3.96 \mathrm{mg}$ ( $0.02 \mathrm{mmol})$ of EGDMA, and $6.57 \mathrm{mg}(0.04 \mathrm{mmol})$ of AIBN were placed in a capillary of $7.0 \mathrm{~mm}$ in diameter and dissolved in benzene adjusted to $1.0 \mathrm{~mL}$. The solution was degassed and polymerized by heating at $60^{\circ} \mathrm{C}$ for $24 \mathrm{~h}$. The feed ratio was adjusted to TFPB:ODA:EGDMA =5:95:1. Gels with low crosslinking densities were prepared in a similar manner by reducing the feed ratios of EGDMA. The formed gels were washed by swelling in benzene for 
$10 \mathrm{~h}$, and then air-dried at room temperature. The sample was cut into cylinders of about $1.0 \mathrm{~mm}$ in length, and the cylinders were dried in vacuo at $40^{\circ} \mathrm{C}$

Synthesis of EG-18 was performed by Ono et al. (2007) at Kyushu University, Japan.

\subsection{Characterization}

The Fourier transform infrared (FTIR) spectra were performed using J asco FT/ IR-4100. Compression strength was measured with Instron 5900 electromechanical system and the compression speed was 0.25 $\mathrm{mm} / \mathrm{min}$. UV-vis spectra were collected using a ThermoSpectronic Aquamate 100 UV-vis Spectrometer.

\subsection{Swelling studies}

Swelling behavior of NG-18 gels was determined with the following solvents of various polarities at $25 \pm 1^{\circ} \mathrm{C}$ using $5 \mathrm{~mL}$ vials: water, DMSO, methanol, ethanol, isopropanol, 1-octanol, acetone, MIBK, acetonitrile, THF, diethylether, dichloromethane, chloroform, carbon tetrachloride, benzene, toluene, hexane, and cyclohexane. The mass of each empty vial was recorded and then a specified amount of dried gel was added to each vial. The vials were weighed and the amount of dried gel was noted ( $\left.\mathrm{W}_{\text {dry }}\right)$. The vials were then filled with a solvent and allowed to equilibrate for 24, 48 and 72 hours. The excess solvent was removed from the vials and the gels were weighed again ( $\left.\mathrm{W}_{\text {wet }}\right)$. The amount of solvent absorbed by the gels was obtained from the difference in weights. The swelling degree (Q) was defined by Equation 1:

$$
\mathrm{Q}=\frac{\mathrm{W}_{\text {wet }}-\mathrm{W}_{\mathrm{dry}}}{\mathrm{W}_{\mathrm{dry}}}
$$

Temperature dependence on swelling degree of NG-18-1\% gel was measured in the above solvents at $20,40,60,(60 \rightarrow) 0$, and $(25 \rightarrow) 0^{\circ} \mathrm{C}$. Here, $(60 \rightarrow) 0^{\circ} \mathrm{C}$ indicates that the sample was heated at $60^{\circ} \mathrm{C}$ to achieve the equilibrium once and then cooled to $0{ }^{\circ} \mathrm{C}$. This was performed to investigate the record of the heating and cooling process. Due to low boiling points, dichloromethane and diethylether were not used at $40^{\circ} \mathrm{C}$; similarly acetone was not used at $60^{\circ} \mathrm{C}$. Likewise, DMSO and cyclohexane were not 
used at $0{ }^{\circ} \mathrm{C}$ due to high freezing points. These values were indicated as $\mathrm{Q}=0$. To understand the kinetics of swelling behavior, the above procedure was followed with several vials and the amount of solvent absorbed was determined at different time intervals.

\subsection{Critical temperature studies}

Critical temperatures were determined by UV-vis spectroscopy. Swollen NG-18 1\% gel in THF was placed in a temperature controlled quartz cell, which was monitored with thermocouples (OMEGA DP462). The transmittance at $700 \mathrm{~nm}$ was measured as a function of temperature by changing temperature at $0.1^{\circ} \mathrm{C} / \mathrm{min}$. While the swollen gel was transparent, the collapsed gel was opaque. The values of critical temperature in the heating and cooling process were obtained from a plot of transmittance versus temperature.

\subsection{Cyclic temperature change test}

Cyclic temperature changes of both swelling degree and transmittance were performed to ascertain the reversibility of the gel. In the swelling test, a piece of NG-18-1\% gel was first placed in THF for $48 \mathrm{~h}$ at $25^{\circ} \mathrm{C}$. Excess THF was removed from the vial, weighed, and the swelling degree was calculated. Then the vial was filled with THF again, placed at $0{ }^{\circ} \mathrm{C}$ for $24 \mathrm{~h}$, and swelling degree was measured by the same procedure. This cycle was repeated five times in total. In the transmittance study, THF swollen gels were placed in a temperature controlled quartz cell. The transmittance at $700 \mathrm{~nm}$ was measured at $25^{\circ} \mathrm{C}$ and $0{ }^{\circ} \mathrm{C}$ alternatively. Each step took about $30 \mathrm{~min}$, and the procedure was repeated for a total of five cycles. The gels achieved equilibrium values at each step in both swelling and transmittance test.

\subsection{Compression strength}

A piece of dried NG-18 1\% and 0.5\% gels was swelled by adding excess toluene or THF. After eliminating extra solvent, compression strength was measured at a compression speed of $0.5 \mathrm{~mm} / \mathrm{min}$.

\subsection{Oil absorption}

Stainless steel metal coupons were washed by acetone and methanol, and dried in vacuo for 72 hours. The coupons were soaked in SAE-30 oil and allowed to drip excess oil for 30 minutes. Half of these coupons were also 
sprayed with alumina powder. The contaminated coupons were immersed in NG-18 gels or toluene for 30 minutes. The percent of oil absorbed was then measured by comparing the weight of each coupon before and after immersion.

Analogous procedure was followed for field samples obtained from a Navel cleaning facility.

\subsection{Grease cleaning}

Metal coupons were prepared according to MIL-PRF-680B Appendix A, and uniformly coated with MIL-PRF-10924 grease. The coupons were immersed in the NG-18 gels, and trichloroethylene until visually clean. The percent of grease absorbed was determined by comparing the weight of each coupon before and after immersion. The cleaning power was also determined by the following equation:

$$
\%=\left(\frac{(100-A)}{100}\right) \times 100
$$

where $\mathrm{A}$ is the average cleaning time of the three tested runs (MIL-PRF680B 2006).

The commercial solvent BioAct MSO was also tested for cleaning power. Appendix A contains supplemental information regarding the solvent process. Appendix B contains the Material Safety Data Sheets (MSDSs) for Imbiber beads, trichloroethylene (TCE), and THF. 


\section{Results and Discussion}

\subsection{Characterization of NG-18 gels}

Figure 4. FIIR spectra of stearylacrylate.

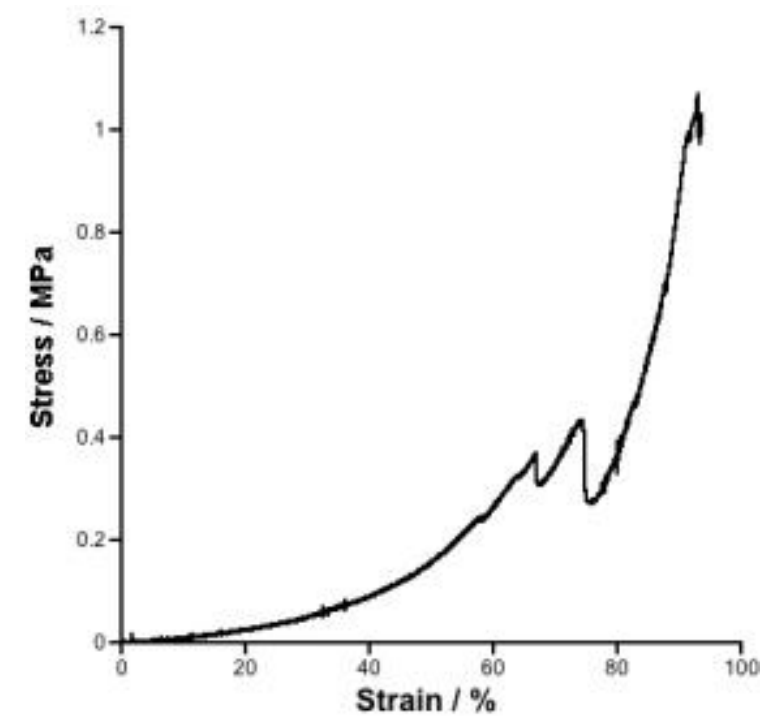

Figure 5. Compression strength of swollen NG-18-1\%, THF has a breaking point around $0.371 \mathrm{MPa}$.

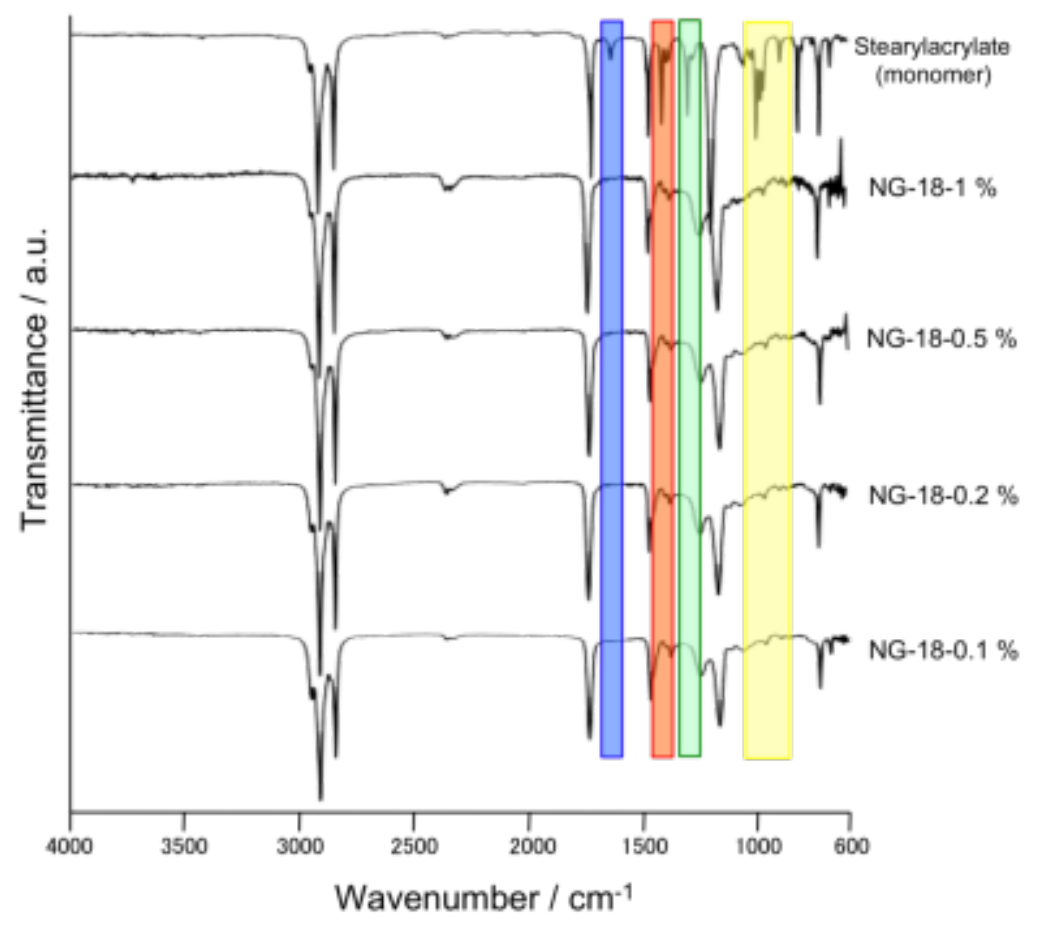


Figure 4 shows FTIR spectra of SA monomer and NG-18-1\%, -0.5\%. Compared to the spectrum of stearylacrylate monomer, NG-18 gels showed the disappearance of peaks in four regions. Each peak was identified as follows: $1634 \mathrm{~cm}^{-1}$ is $\mathrm{C}=\mathrm{C}$ bond vibration, $1410 \mathrm{~cm}^{-1}$ is $\mathrm{C}-\mathrm{H}$ of $\mathrm{C}=\mathrm{CH}_{2}$ in-plane scissoring, $1297 \mathrm{~cm}^{-1}$ is $\mathrm{C}-\mathrm{H}$ of $\mathrm{C}=\mathrm{CH}$ in-plane vibration and 997 and 893 $\mathrm{cm}^{-1}$ are $\mathrm{C}=\mathrm{CH}$ out-plane vibration. The disappearance of these peaks of the vinyl group indicates that NG-18 gels include a little non-reacted SA. Figure 5 shows the compression strength of swollen NG-18-1\% gel in THF. The first breaking point is the stress of $0.371 \mathrm{MPa}$ and the fracture strain of the gel is $\lambda=67 \%$. The NG-18-1\% gel could withstand a similar degree of compression as reported by single network poly acrylamide gel prepared by Gong et al. (2003).

\subsection{Swelling behavior}

The swelling behavior of NG-18 gels (NG-18-1\% and -0.5\%) in the solvents with various polarities from cyclohexane to water at $25^{\circ} \mathrm{C}$ was investigated (Figure 6). The swelling degree increased with increasing polarity from cyclohexane, and the maximum value was observed in chloroform. On the other hand, the gels collapsed in the more polar solvents (dielectric constant $\varepsilon>10$ ). Particularly, NG-18 gels absorbed large amounts of chlorinated solvents such as chloroform ( $\mathrm{Q}=35$ on NG-18-1\%) and carbon tetrachloride $(\mathrm{Q}=36)$. Moreover, NG-18 swelled in a moderate amount of solvents such as ether (diethylether: $\mathrm{Q}=12$, THF: $\mathrm{Q}=17$ ), aromatic compounds (benzene: $\mathrm{Q}=21$, toluene: $\mathrm{Q}=22$ ), and aliphatic reagents (hexane: $\mathrm{Q}=14$, cyclohexane: $\mathrm{Q}=20$ ). In more polar solvents, such as water, DMSO, alcohols (methanol, ethanol, isopropanol, and 1-octanol), ketones (acetone and MIBK), and acetonitrile, NG-18 did not swell at all (Q<1). Also, enhancing swelling ability by reducing the cross linker density was attempted. Reducing the feed ratio of the crosslinker to the monomer from $1 \mathrm{~mol} \%$ to $0.1 \mathrm{~mol} \%$ enhanced the swelling ratio. However, the gels less than 0.2 mol\% crosslinker densities were too soft to separate excess solvent and swelling degree could not be accurately measured. NG-18-0.5\% indicated the same tendency as NG-18-1\% and had a higher swelling degree than NG-18-1\%. These swelling behaviors of NG-18 gels essentially depend on the compatibility of the polymer chain with the media. NG-18 did not allow penetration of the highly polar molecules into the polymer networks, while non-polar solvents were absorbed. 
Figure 6. Swelling degree (Q) of NG-18-1\%, $-0.5 \%$ and imbiber beads in various solvents.

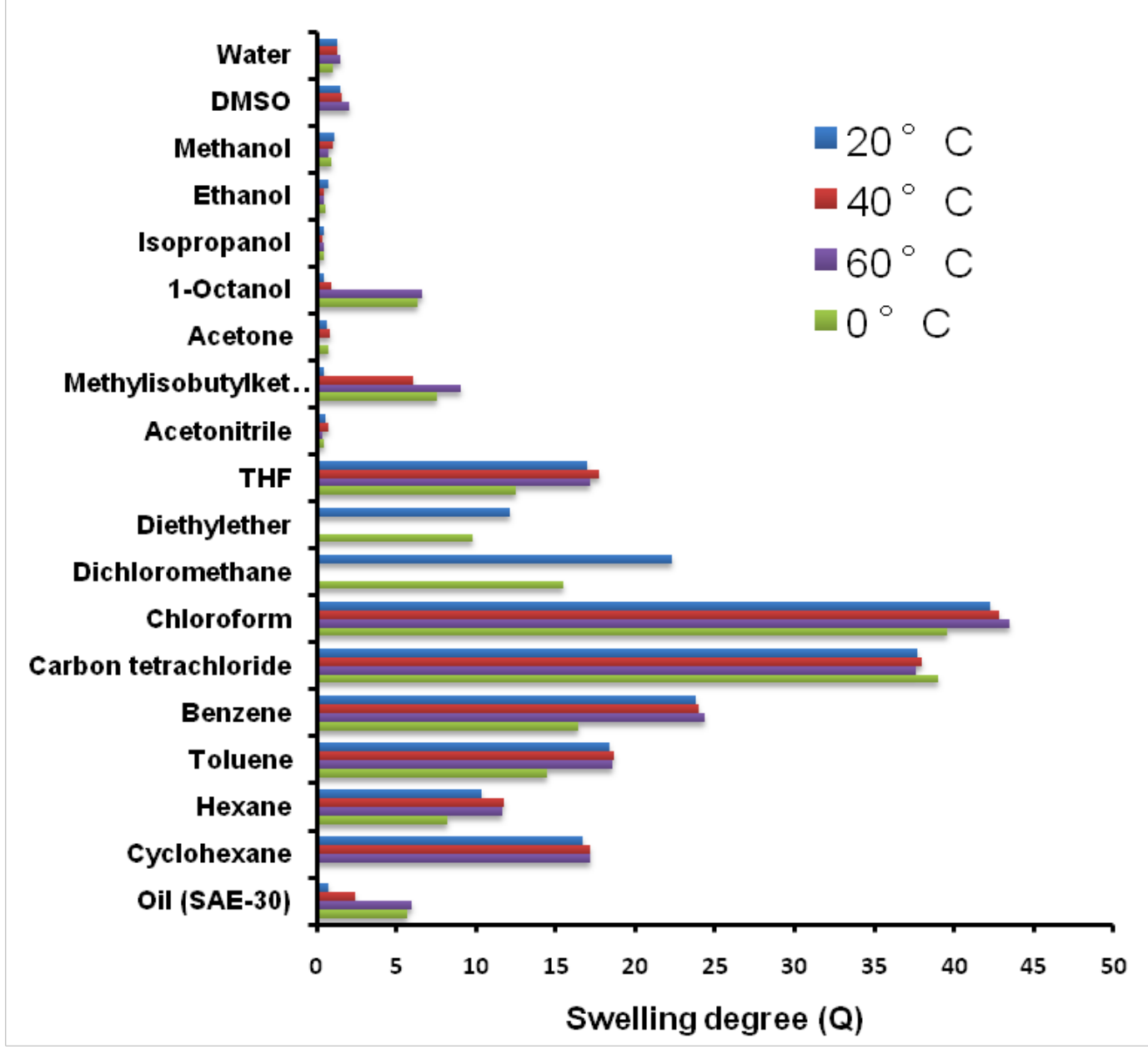

Figure 7 shows temperature dependence on the swelling degree with NG$18-1 \%$ gel. The temperature was changed from $20^{\circ} \mathrm{C}$ to 40,60 , and $0{ }^{\circ} \mathrm{C}$ successively, and the variety of solvents is the same as ones used in swelling degree test at $25^{\circ} \mathrm{C}$. Also, the sample cooled from $25^{\circ} \mathrm{C}$ to $0{ }^{\circ} \mathrm{C}$ was studied to investigate the influence of thermal record during the heating process for swelling behavior, which is represented as $(25 \rightarrow) 0^{\circ} \mathrm{C}$. Figure 8 summarizes a comparison between the $(60 \rightarrow) 0{ }^{\circ} \mathrm{C}$ and $(25 \rightarrow) 0{ }^{\circ} \mathrm{C}$ conditions. 
Figure 7. Temperature dependence of swelling degree (Q) of NG-18-1\% in various solvents.

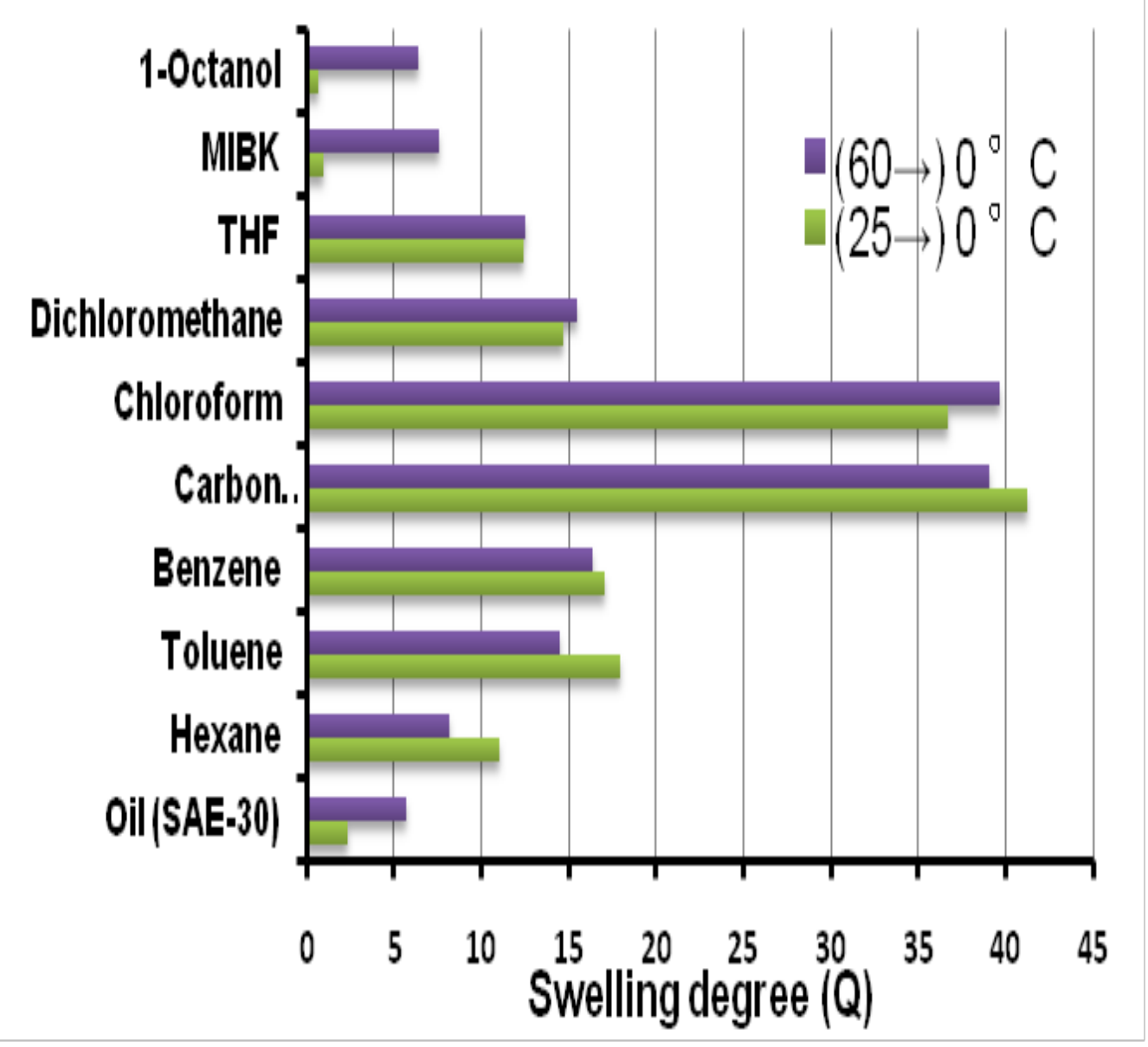

The results in Figure 7 were categorized as:

1. In the following solvents, swelling degree did not change in both heating and cooling processes: water, DMSO, methanol, ethanol, isopropanol, acetone, acetonitrile, carbon tetrachloride, and cyclohexane.

2. Whereas the $Q$ value did not change by heating a maximum of $25 \mathrm{ut} \%$ of the following solvents was dislodged from the gel in cooling process: THF, diethylether, dichloromethane, chlonoform, benzene, toluene, and hexane.

3. The swelling degree increased by heating, but was almost the same by cooling in the solvents 1-octanol and MIBK. The second category is especially remarkable because it showed the changes of swelling degree and the color change from transparent to opaque by cooling to $0{ }^{\circ} \mathrm{C}$. These transition behaviors are attributable to crystallization of long-alkyl chain among stearylacrylate. It is expected that this ability can be used to develop a VOC recycling system composed of both uptake and ejection. On the other hand, the transitions in the third category were irreversible (Figure 8). 
Figure 8. Comparison of heated record of NG-18 gels in the cooling process.

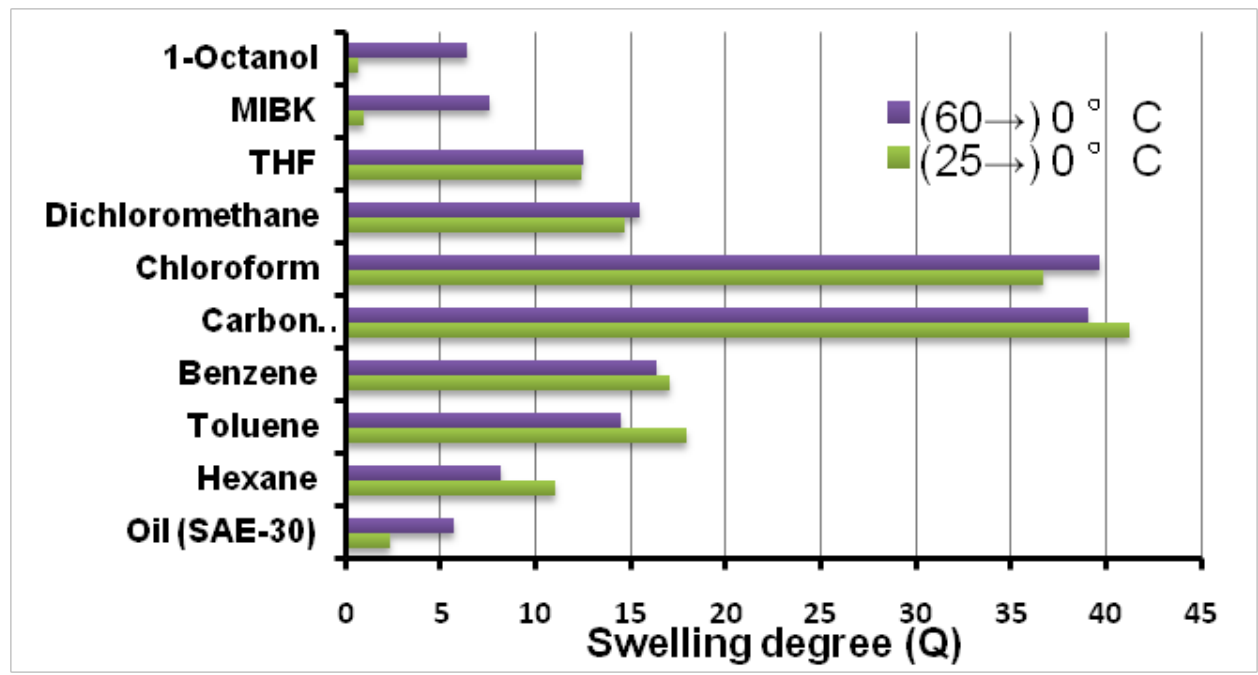

Additionally, to determine the time dependence on the swelling degree of NG-18 gels in THF, the swelling ratio was determined as a function of time. Figure 9 shows the time required for each gel to reach the equilibrium swelling degree. A cubic dry gel (NG-18-1\%, -0.5\%), $5 \mathrm{~mm}$ on a side, was placed in a vial with excess THF at $25^{\circ} \mathrm{C}$.

The kinetics of the swelling behavior was examined by fitting the data to Lagergren pseudo-first and pseudo second order kinetic equations (Kiyonaka et al. 2004, MIL-PRF-680B 2006, and Boddu 2011):

$$
\begin{gathered}
\frac{d q_{t}}{d t}=k_{1}\left(q_{e}-q_{t}\right) \\
\frac{d q_{t}}{d t}=k_{1}\left(q_{e}-q_{t}\right)
\end{gathered}
$$

The values of the first and second order rate constants obtained through the linearization of equation 2 and 3 are included in Table 1, along with the values of regression coefficients. A second-order kinetic equation better describes the swelling behavior of NG-18 gels.

Table 1. Lagergren first and second rate constants (k1 and k2) for swelling of the NG-18 gels

\begin{tabular}{|l|l|l|l|l|}
\hline & \multicolumn{2}{|c|}{ First order } & \multicolumn{2}{c|}{ Second order } \\
\hline Sample & $k_{1} \mathrm{~min}^{-1}$ & $\mathrm{R}^{2}$ & $\begin{array}{l}\mathrm{k}_{2}, \mathrm{~g} \mathrm{~g}^{-1} \mathrm{~min}^{-} \\
1\end{array}$ & $\mathrm{R}^{2}$ \\
\hline NG-18 1\% & $4.38 \times 10^{-3}$ & 0.971 & $5.55 \times 10^{-4}$ & 0.998 \\
\hline
\end{tabular}




\begin{tabular}{|l|l|l|l|l|}
\hline & \multicolumn{2}{|c|}{ First order } & \multicolumn{2}{c|}{ Second order } \\
\hline NG-18 0.5\% & $5.30 \times 10^{-3}$ & 0.985 & $5.72 \times 10^{-4}$ & 0.999 \\
\hline
\end{tabular}

Figure 9. Swelling degree changes of NG-18 with time in THF.

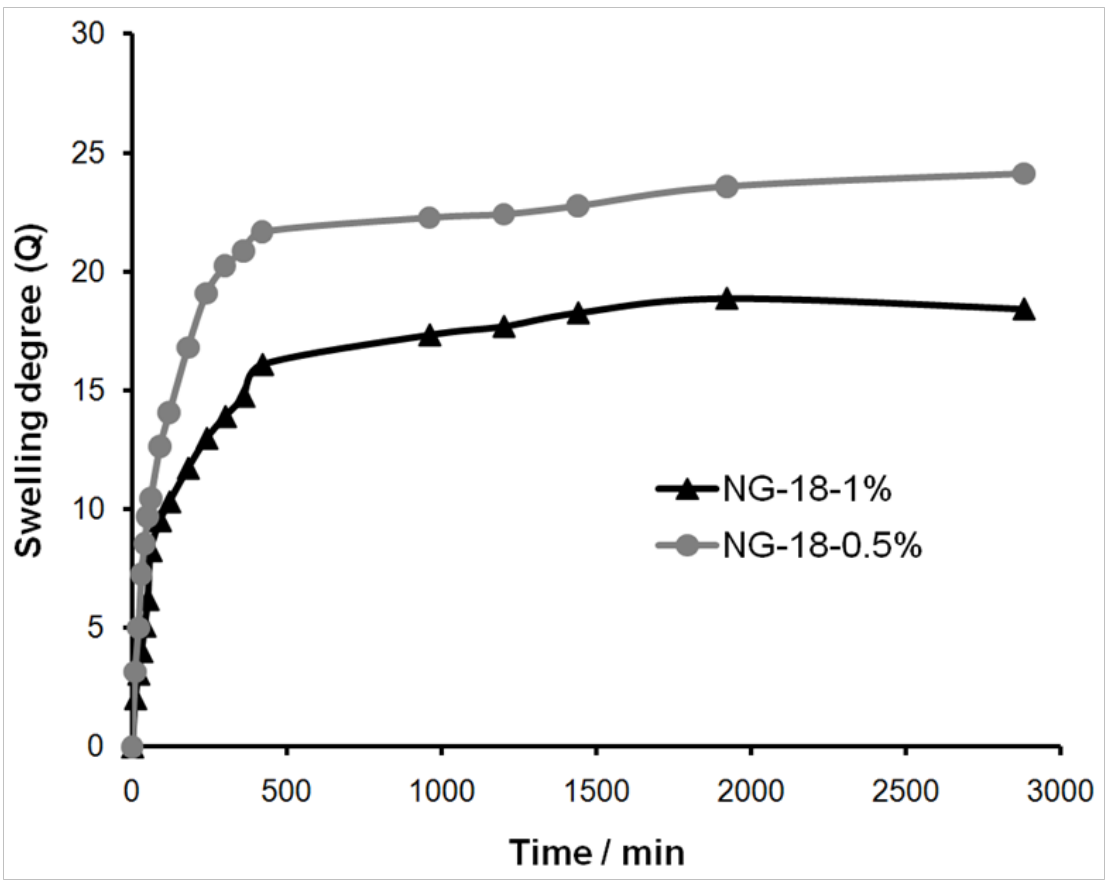

\subsection{Critical solution temperature}

Critical solution temperature was determined for swollen NG-18-1\% in THF. Figure 10 shows the result obtained at $700 \mathrm{~nm}$. The swollen gel is relatively transparent, while the collapsed gel is opaque. Thus, the transmittance values sharply change when the gel is collapsed. The transmittance values were plotted against temperature to obtain approximate critical temperature in both the heating and cooling processes. Transition temperature results in $6.6^{\circ} \mathrm{C}$ in the cooling process and $12.4^{\circ} \mathrm{C}$ in the heating process. This hysteresis was due to supercooling phenomenon on the cooling process. This transition process is different from NIPAM $(\mathrm{N}-$ isopropylacrylamide) in water, depending on the crystallization of long alkyl chain among stearylacrylate unit. 
Figure 10. (a) Temperature dependence of transmittance at $700 \mathrm{~nm}$ of NG-18-1\% gel swelled in THF. (b) Photograph of transparent state at $25^{\circ} \mathrm{C}$. (c) Photograph of opaque state at $0{ }^{\circ} \mathrm{C}$.

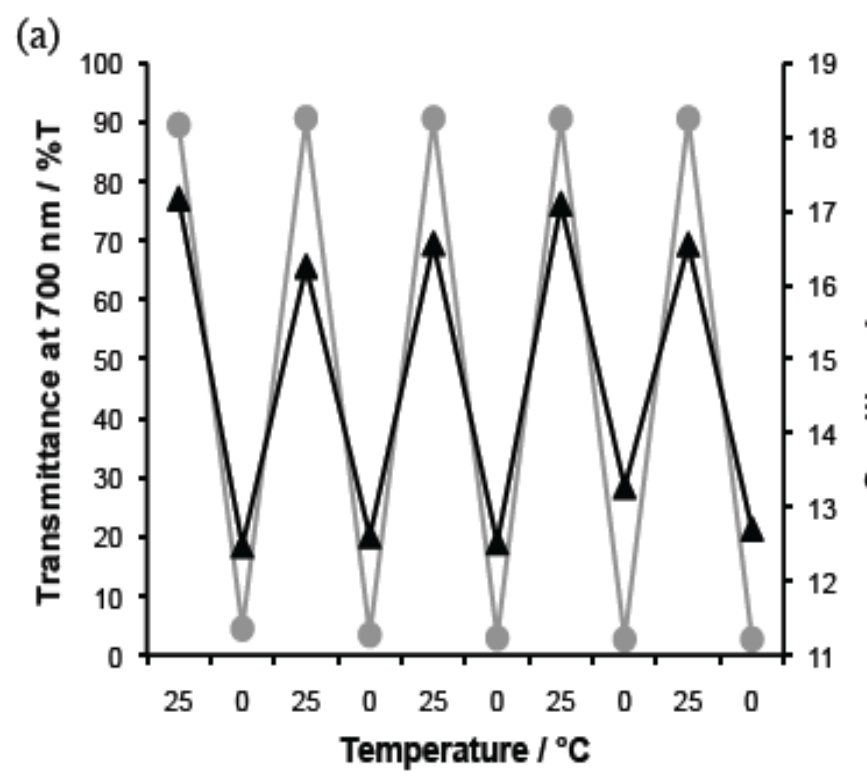

(b)

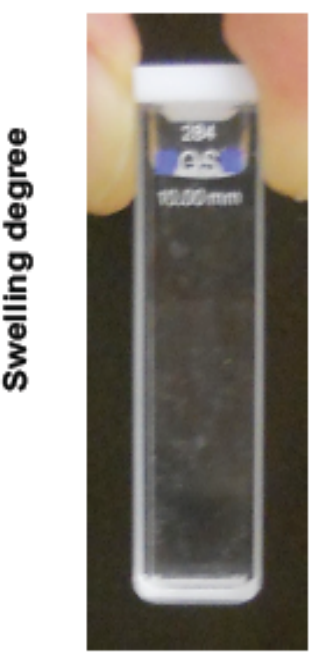

(c)

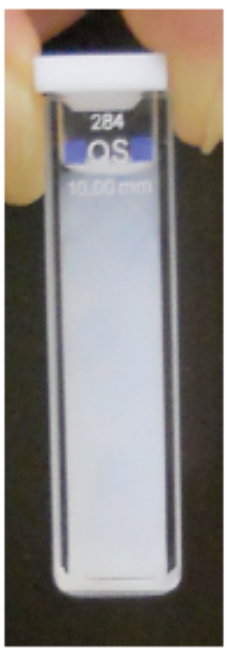

\subsubsection{Cyclic temperature changes test}

The cyclic swelling degree and transmittance studies were performed to investigate the reversibility and reproducibility of the swelling behavior. Figure 11 shows the procedures followed for five cycles for NG-18-1\% gels and the results. The gel appears to be stable and retains its transition characteristics even after five cycles. In other words, this transition is reversible and non-destructive for the gel network. The change of transmittance is very fast and the color change occurs in less than $30 \mathrm{~min}$, but the change of swelling degree was slow, taking more than $24 \mathrm{~h}$. This means that the color change is part of the swelling process but the color change is not equivalent to the change of swelling degree.

\subsubsection{Compression studies}

The first breaking point is $0.167 \mathrm{~N}$ in NG-18- $1 \%$ and about $1 \mathrm{~N}$ in NG-180.5\%. Compared to EG-18, NG-18 gels are much stronger (Figure 12). 
Figure 11. (a) Results of temperature cycle $\left(25^{\circ} \mathrm{C} \rightarrow 0{ }^{\circ} \mathrm{C}\right)$ test: changes of (circle) transmittance at $700 \mathrm{~nm}$, (triangle) swelling degree in THF. (b) Photograph of transparent state at 9th step. (c) Photograph of opaque state at 10th step.

(a)

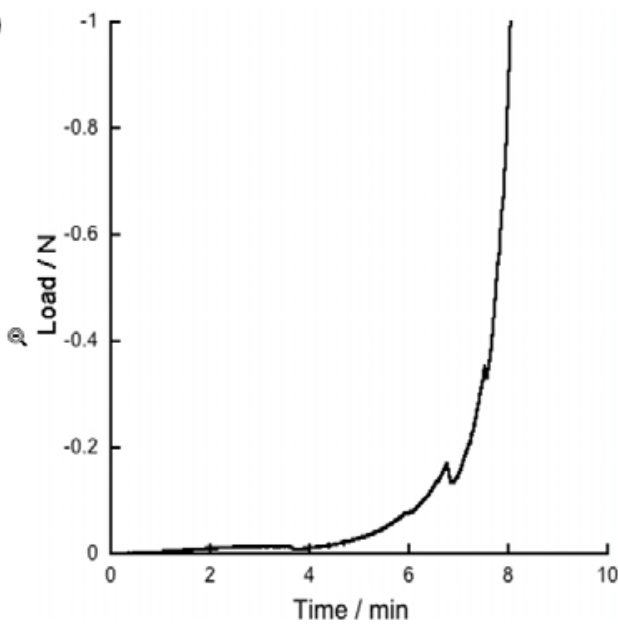

(b)

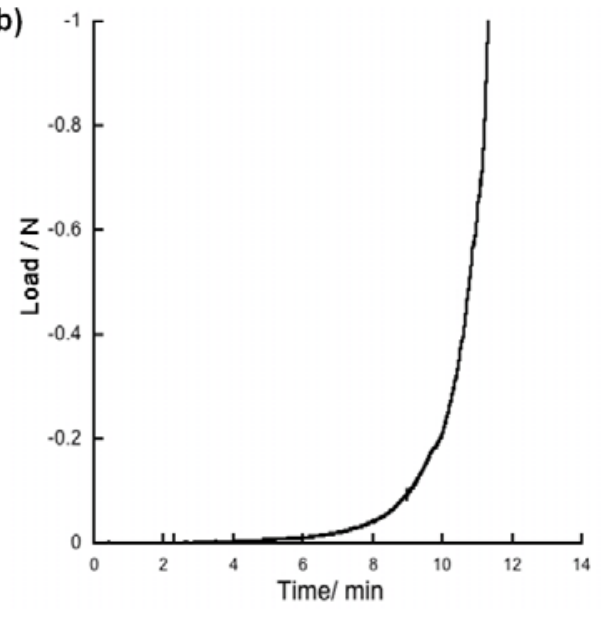

Figure 12. Compression strength of swollen (a) NG-18-1\% and (b) NG-18-0.5\% in toluene.

(a)

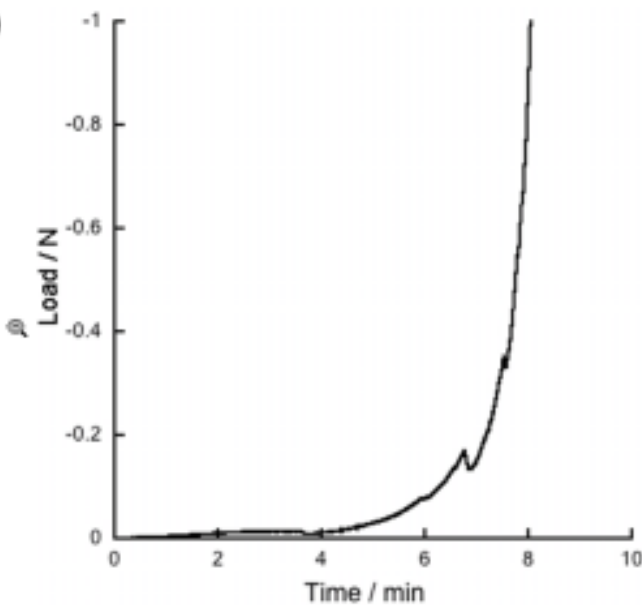

(b)

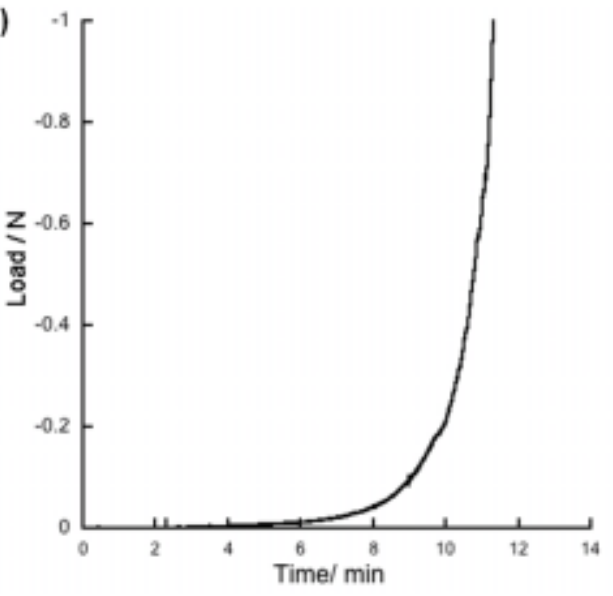

\subsubsection{Oil absorption}

Oil absorption was tested with both NG-18 gels in toluene and in THF. Figures 13 and 14 show the results. It was determined that NG-18 1\% in THF was the best performer in this category because of its high swelling degree, good recyclability, the high oil and alumina absorption properties (Figure 15). THF is also a low- or non-toxic and environmentally friendly solvent. NG-18 0.5\% gel was not able to clean all of the alumina powder and oil. Along with metal coupons, NG-18 gels were tested on painted coupons and stainless steel parts with bolts, the results of which are shown in the appendix. The painted coupons did not show any signs of peeling, and the bolts and flat coupons were cleaned almost as well as using solvent by 
itself. From these data an oil recycling system (Figure 16) was made, which can recycle up to $25 \%$ of solvent when cooled to $0^{\circ} \mathrm{C}$.

Temperature did change the swelling degree of NG-18-1\% in 1-Octanol, methylisobutylketone, and SAE-30 oil when heating from $20^{\circ} \mathrm{C}$ to $40{ }^{\circ} \mathrm{C}$ or $60{ }^{\circ} \mathrm{C}$ (Figure 7). Even if they were cooled to $0^{\circ} \mathrm{C}$, however, they did not collapse. In other words, these changes were irreversible.

Figure 13. Oil absorption properties of NG-18 gels.

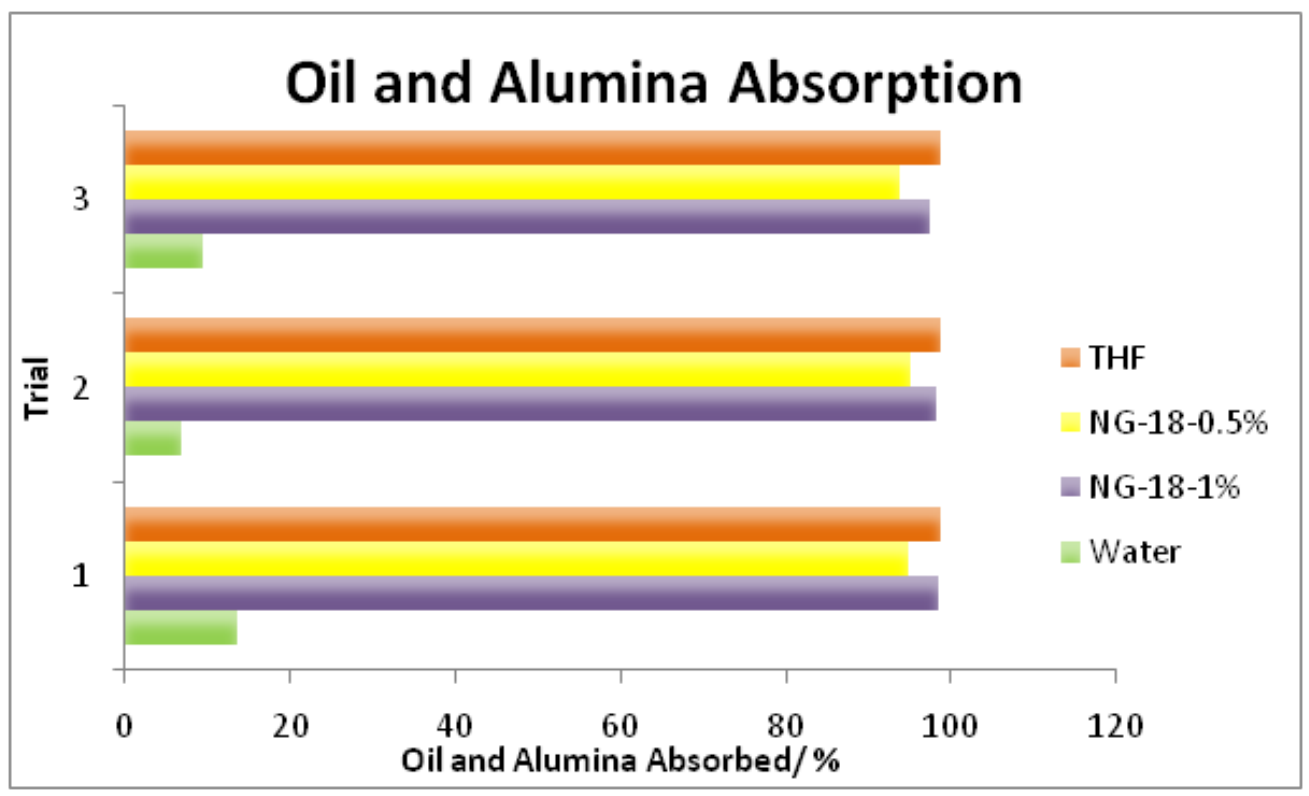

Figure 14. Metal surface cleaning properties of NG-18 gels.
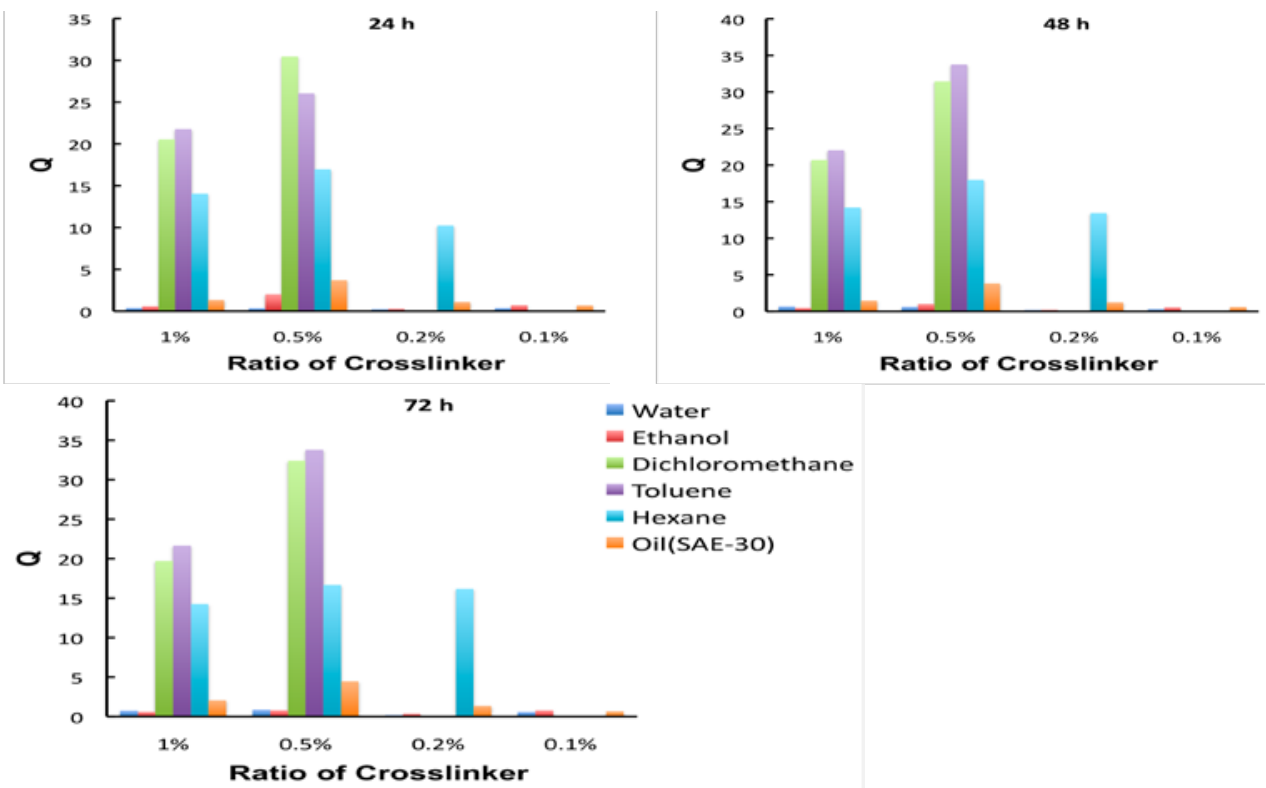

- Water

Ethanol

Dichloromethane

Toluene

Oil(SAE-30) 
Figure 15. Swelling degree of NG-18-x\% in various solvents after (a) $24 \mathrm{~h}$, (b) $48 \mathrm{~h}$, and (c) $72 \mathrm{~h}$.

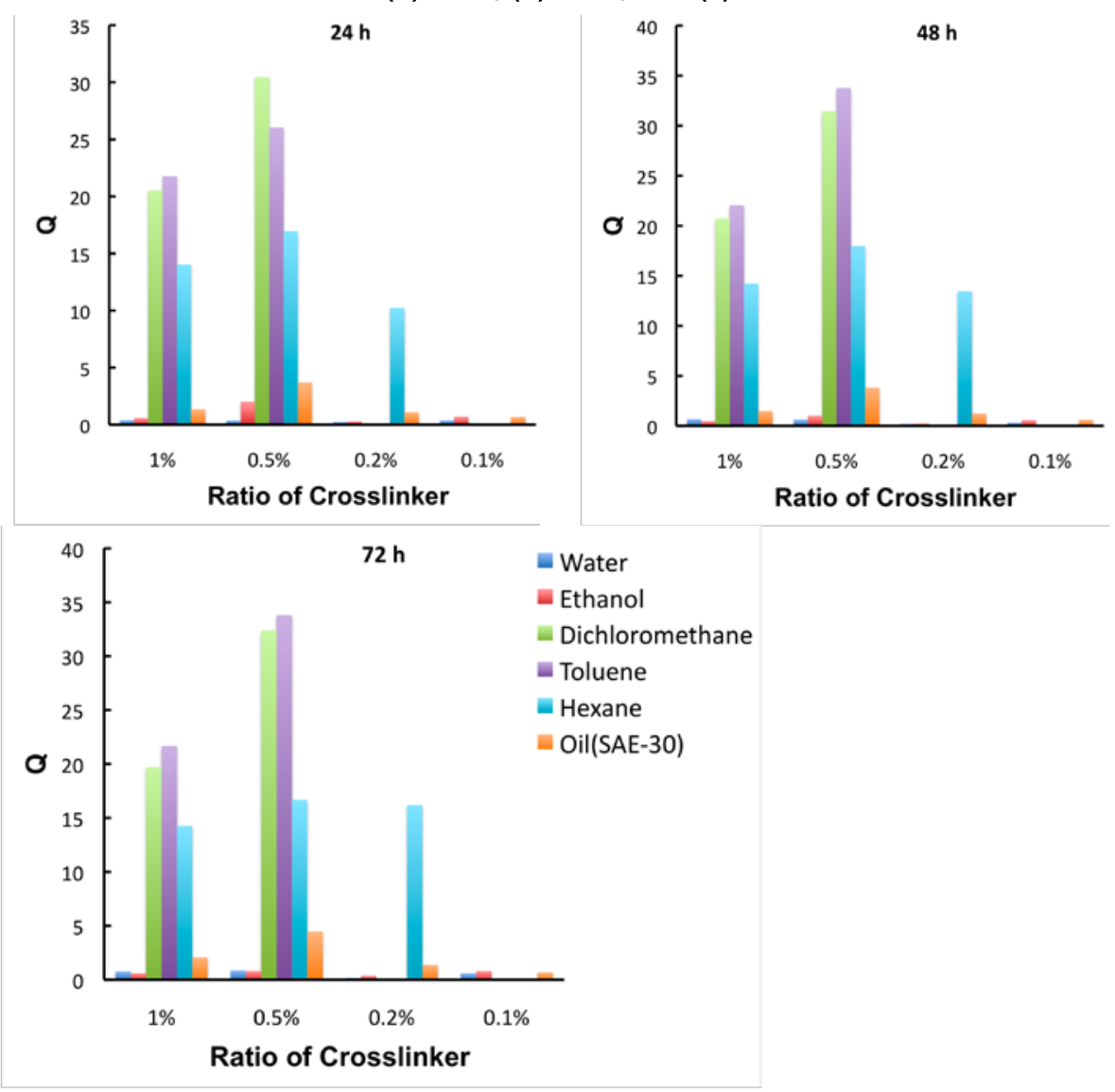

Figure 16. Oil recycling system with swollen NG-18-1\% gel in THF.
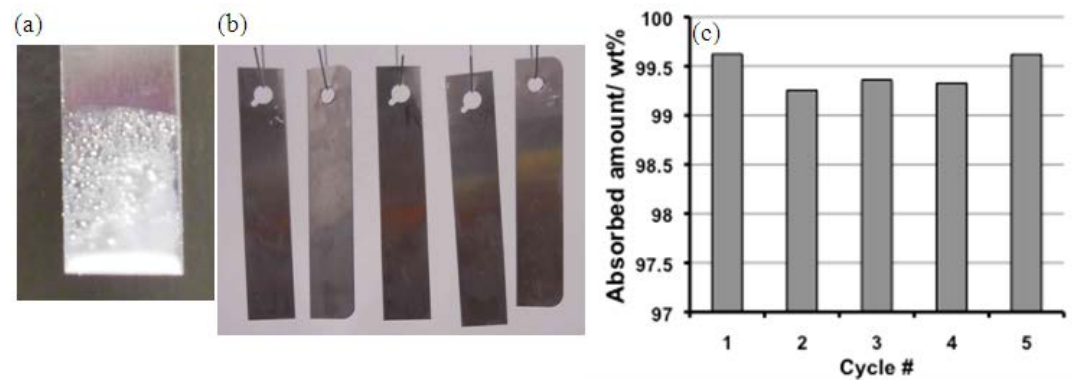

The recyclability of NG 18 1\% gel for surface cleaning is illustrated in Figure 16. This system is pending patent application (Boddu 2011). The recyclability was tested by five consecutive oil absorption-desorption processes (Figures 17 and 18). The swelling degree of the gel in each step was not measured because the initial weight of the gel can change due to addition of oil and particulates. Instead, the amount of absorbed oil squeezed out by the collapsed gel was weighed in each step. The weight of the particulates and THF were accounted for by filtration, and evaporation, respectively. 
Figure 17. Photographs of metal coupons (a) before and (b) after immersing the gel: from left side, cycle1-5. (c) Absorbed amount in each cycle.
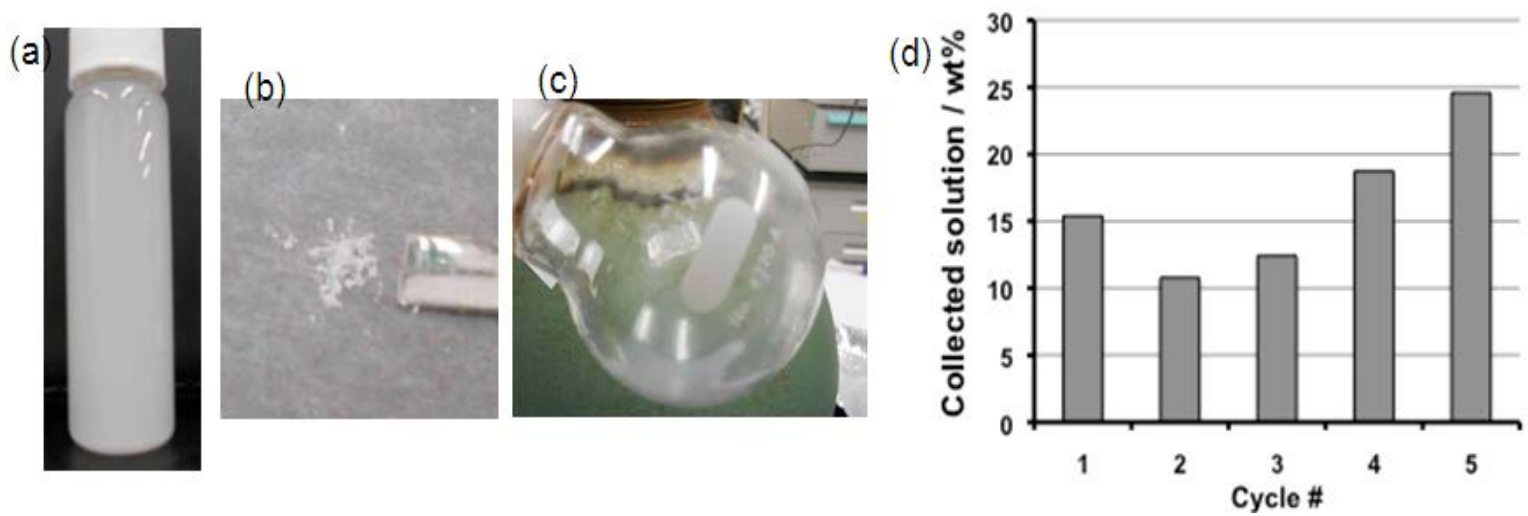

Figure 18. Photographs of (a) collected solution, (b) filtrated residual particulates, and (c) residual oil after evaporation. (d) Ratio of collected solution amount for the weight of gel on each cycle.
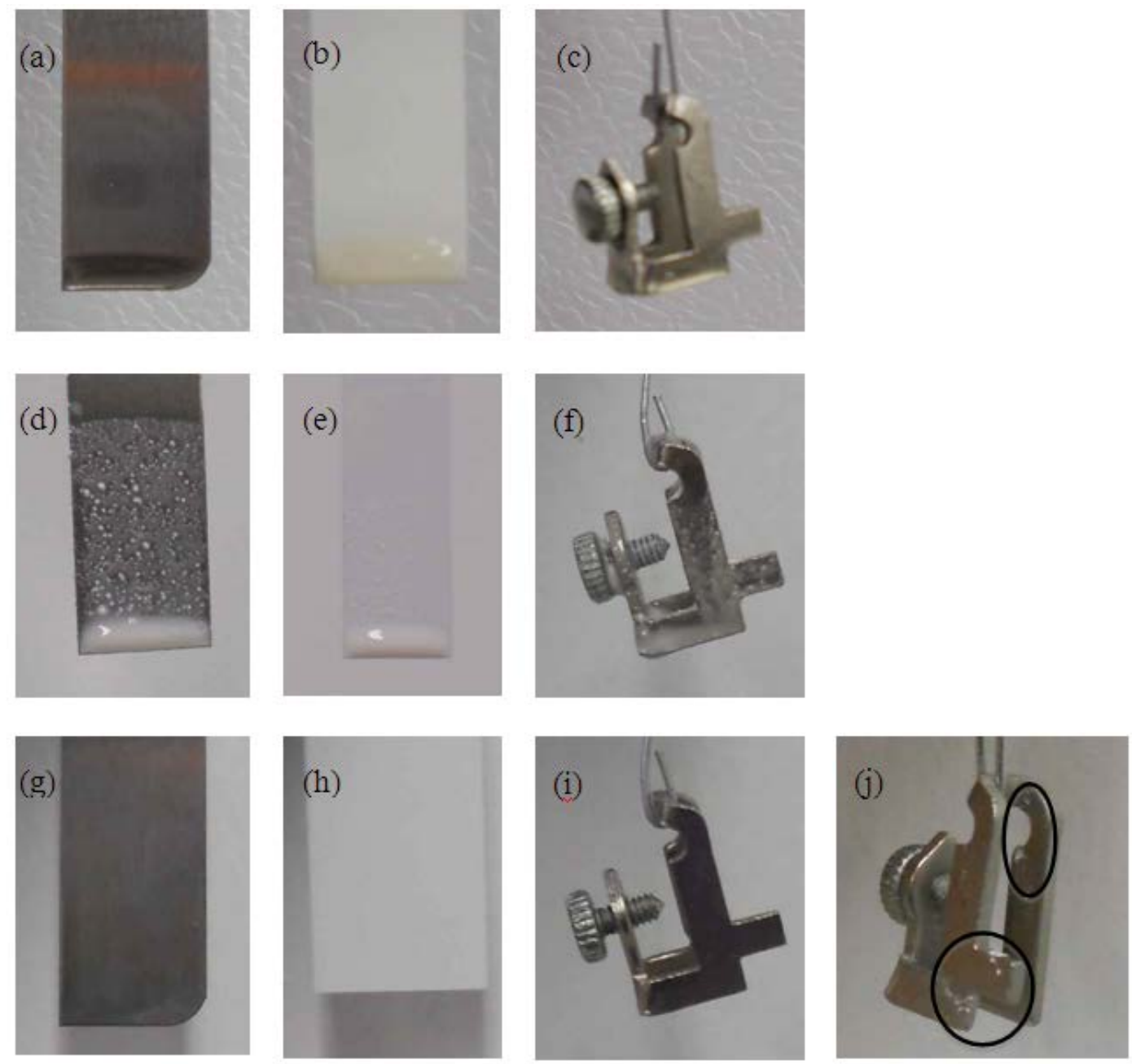
The cleaning property of NG-18 1\% gel was maintained at >99 wt\% even after five cleaning cycles. By cooling, a solution of particulate, THF, and oil was removed from the gel. The particles were removed by filtration, and 10-25\% THF could be evaporated, recollected and recycled. The distribution of the amount of collected solution was attributed to the size of the gel, so as the number of cycles increased, so did the number of solutions collected.

In addition to testing preformed on metal coupons, materials from a Naval cleaning facility were tested as shown in Figures 19 and 20.

Figure 19. Photographs of metal coupons (a)-(c) soaked in SAE-30 oil, (d)-(f) soaked in the mixture of SAE-30 oil and alumina powder, (g)-(i) immersed in NG-18-1\% gel, and (j) immersed in NG-18-0.5\% gel (circles indicate the alumina remaining area).

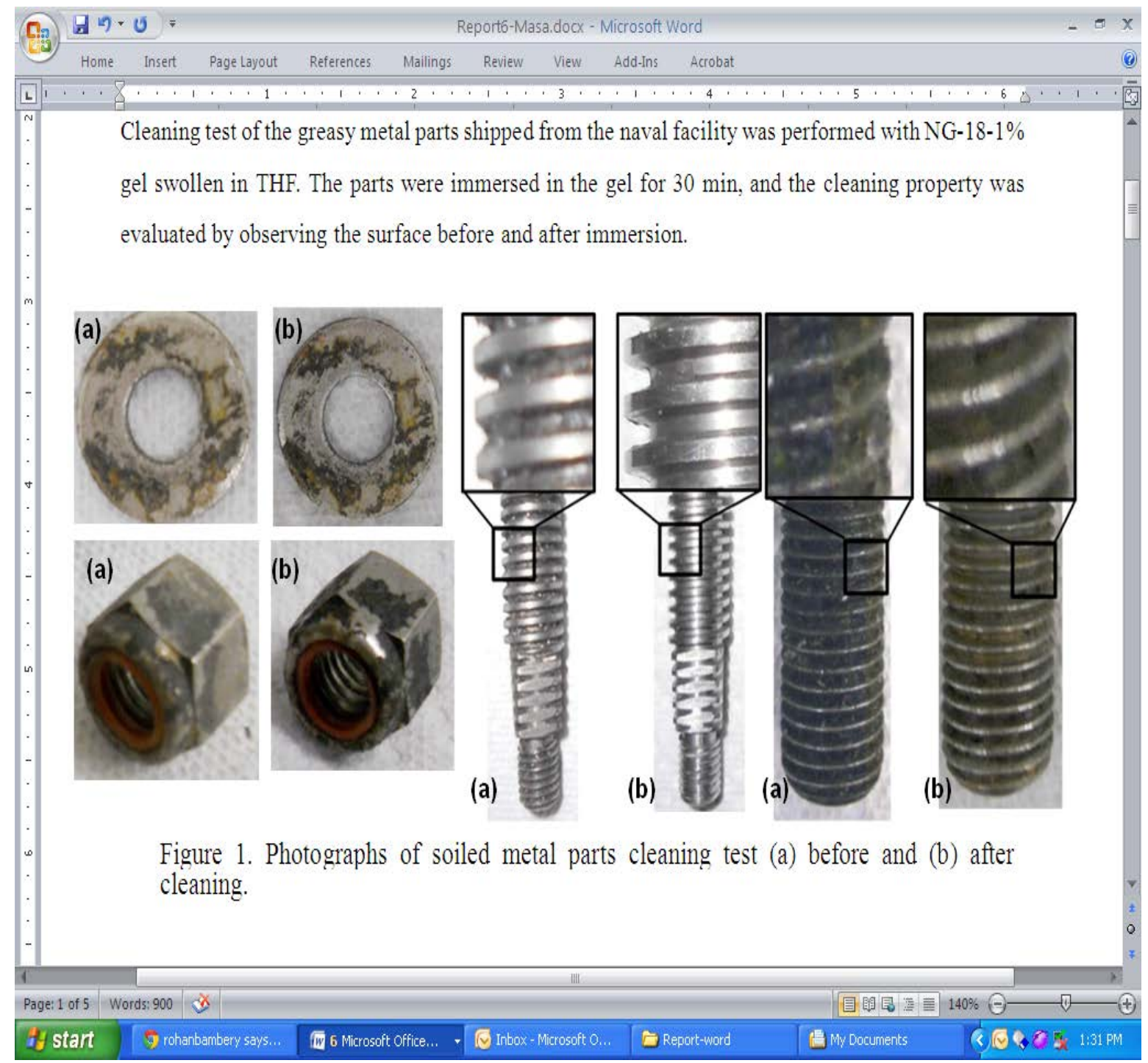


Figure 20. Photographs of soiled metal parts cleaning test (a) before and (b) after cleaning.

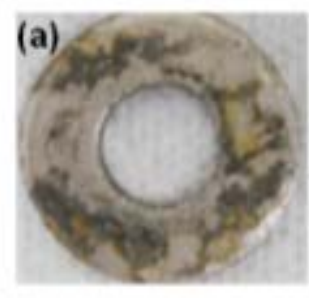

(a)

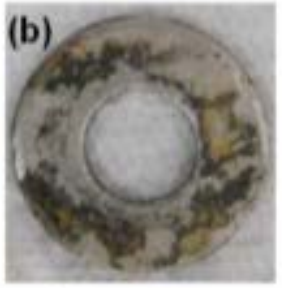

(b) (a)

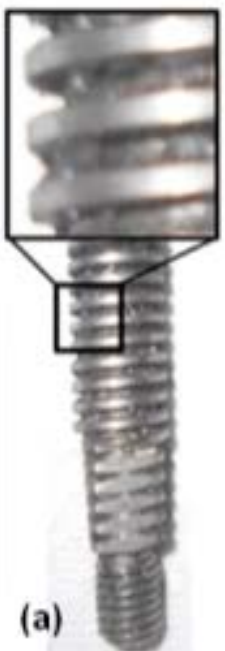

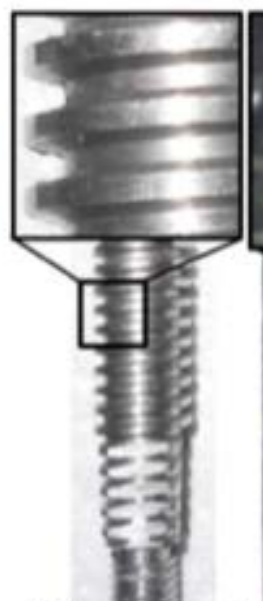

(b)

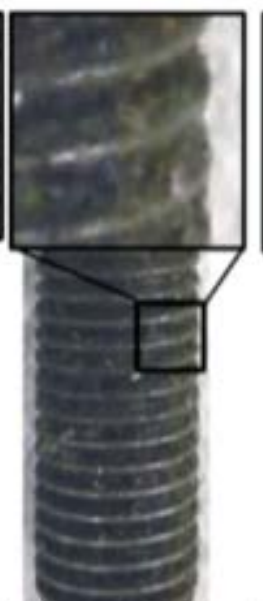

(a)

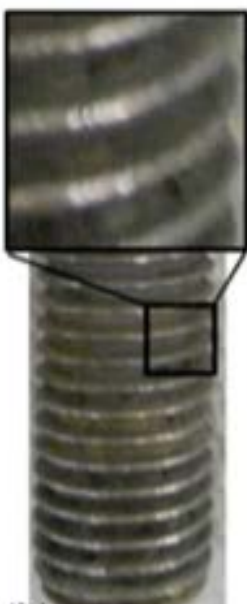

(b)

\subsection{Grease cleaning}

The grease absorption capabilities of NG-18 gels were nearly as good as trichloroethylene all showing greater than $99 \%$ absorption capabilities. As shown in Figures 21 and 22, NG-18 0.5\% outperformed the TCE in two of three trials. However, the NG-18 0.5\% also had an ability to slightly damage the metal as in Trial 1. NG-18 1\% was the worst of the three tested, though it showed a higher cleaning power than NG-18 0.5\%. This could be due to the tendency of the NG-18 1\% to stay on the metal after removing the grease. This could be prevented by washing with ethanol immediately after cleaning.

Figure 21. Grease absorption properties of NG-18 gels.

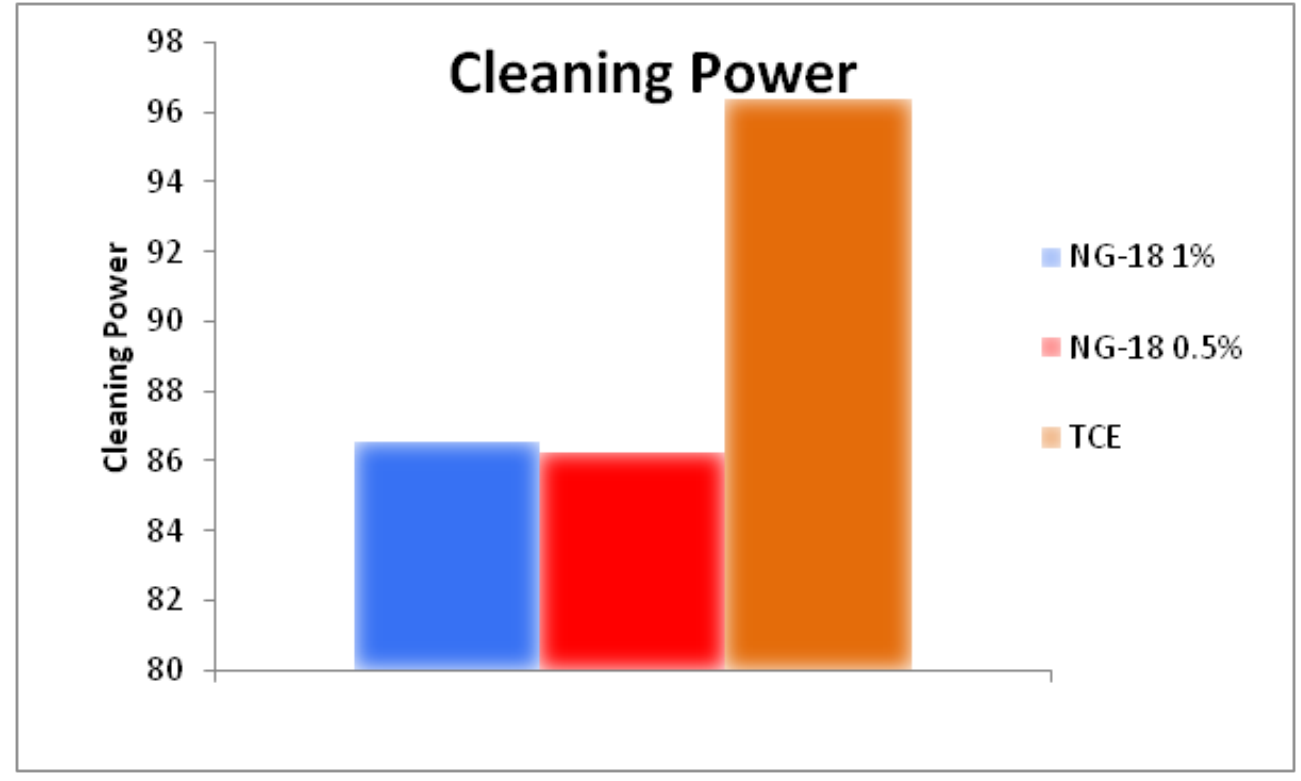


Figure 22. Cleaning power of NG-18 gels.

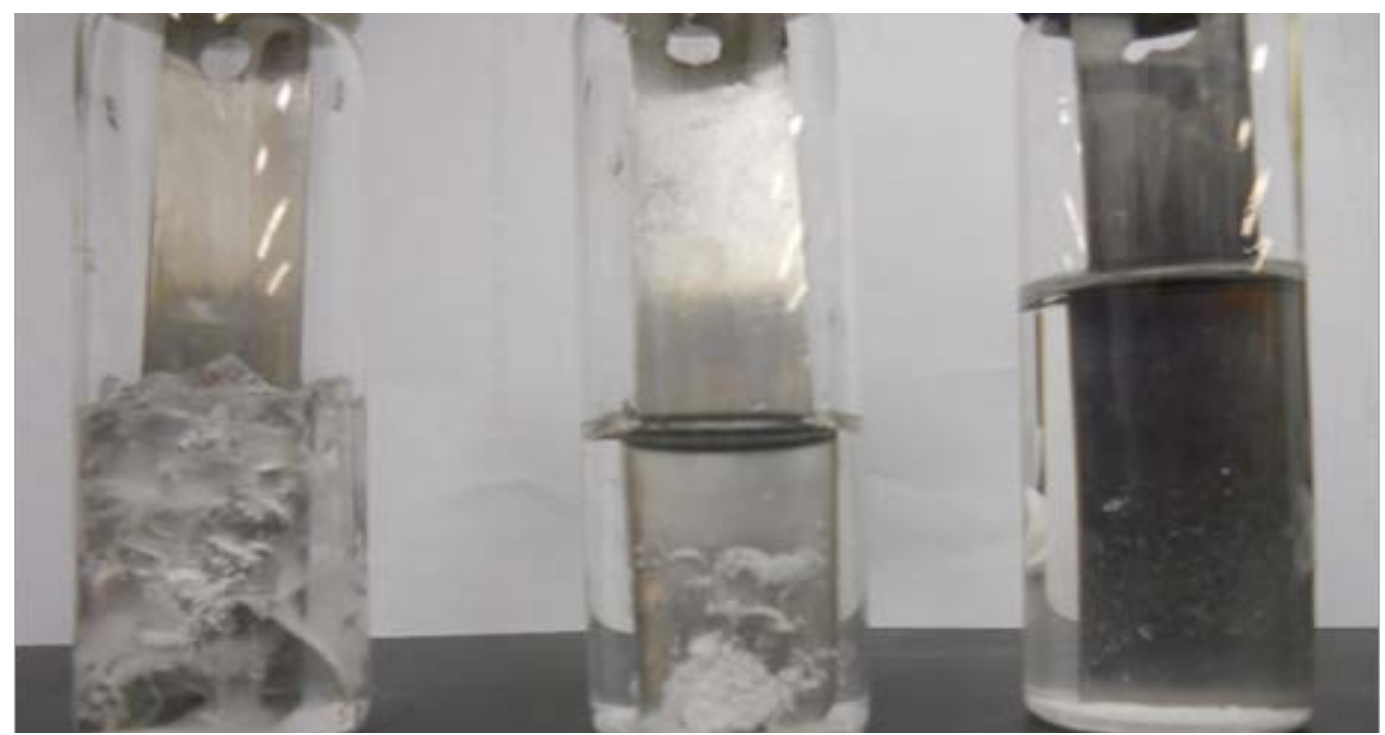

\subsection{Cost assessment}

\subsubsection{Cost reporting}

The objective of this project was to evaluate and demonstrate NG-18 gels as replacements for MIL-PRF-680 solvents for use on weapons systems and platforms. Implementing these processes would require minimal capital investment, as most facilities already have cleaning tanks on hand. The only substantial investment would be in production of the gel and solvent use. Existing equipment would not need to be replaced.

The cost of production was analyzed based on a 10x4x4-ft (3400 L) cleaning tank filled to various degrees, assuming no solvent loss between cleanings, and a 250-day work year accounting for holidays and weekends.

For comparison of costs of solvent cleaning versus the gel cleaning system, the following assumptions were made:

1. The solvent could be used till it is saturated with oil, grease, and dirt. The solvent would be used for three cycles before the solvent is replaced.

2. The gel system is used for eight solvent cycles.

The cost analysis is for comparing the two cleaning approaches. With an optimized system the absolute costs for each may be different. Tables 2-5 show the amounts of chemicals used and overall costs of each cleaning system. From this analysis it was determined that the cost of the gel system is less than that of the current cleaning system. 


\subsubsection{Cost analysis}

Table 2. Chemical cost and amounts needed to make one kilogram of dry NG-18 gel.

\begin{tabular}{|c|c|c|c|c|c|c|c|}
\hline & & & & \multirow{2}{*}{\multicolumn{2}{|c|}{ NG-18 1\% }} & & \\
\hline & & & & & & \multicolumn{2}{|l|}{ NG-18 0.5\% } \\
\hline Chemical & $\begin{array}{l}\text { Price per } \\
\text { unit (\$) }\end{array}$ & $\begin{array}{l}\text { Amount } \\
\text { per unit }\end{array}$ & $\begin{array}{l}\text { Number of } \\
\text { Units } \\
\text { required }\end{array}$ & $\begin{array}{l}\text { Amount } \\
\text { Needed }\end{array}$ & $\begin{array}{l}\text { Cost } \\
(\$)\end{array}$ & $\begin{array}{l}\text { Amount } \\
\text { Needed }\end{array}$ & Cost (\$) \\
\hline Benzene & 67.00 & $1 \mathrm{~L}$ & 1 & $236.1 \mathrm{~mL}$ & 67.00 & $225.28 \mathrm{~mL}$ & 67.00 \\
\hline $\mathrm{SA}$ & 89.20 & $1 \mathrm{~kg}$ & 2 & $1.1806 \mathrm{~kg}$ & 178.40 & $1.2642 \mathrm{~kg}$ & 178.40 \\
\hline EGDMA & 33.80 & $100 \mathrm{~g}$ & 1 & $7.2 \mathrm{~g}$ & 33.80 & $3.86 \mathrm{~g}$ & 33.80 \\
\hline AlBN & 30.60 & $25 g$ & 1 & $11.9 \mathrm{~g}$ & 30.60 & $12.8 \mathrm{~g}$ & 30.60 \\
\hline Hexane & 349.20 & $\begin{array}{l}208.198 \text { L (55 } \\
\text { gal) }\end{array}$ & 1 & $30 \mathrm{~L}$ & 349.20 & $30 \mathrm{~L}$ & 349.20 \\
\hline & & & & Total Cost & 659.00 & Total Cost & 659.00 \\
\hline
\end{tabular}

Table 3. Chemical amounts of NG-18 and trichloroethylene needed to fill a $3400 \mathrm{~L}$ cleaning tank to various levels

\begin{tabular}{|c|c|c|c|c|c|c|c|c|c|c|c|c|}
\hline \multicolumn{13}{|l|}{ NG-18 1\% } \\
\hline \multirow[b]{2}{*}{ Chemical } & \multicolumn{12}{|c|}{ Amount needed per fill height } \\
\hline & \multicolumn{2}{|l|}{$100 \%$} & \multicolumn{2}{|l|}{$75 \%$} & \multicolumn{2}{|l|}{$67 \%$} & \multicolumn{2}{|l|}{$50 \%$} & \multicolumn{2}{|l|}{$33 \%$} & \multicolumn{2}{|l|}{$25 \%$} \\
\hline Benzene & 5.352 & $L$ & 4.014 & $\mathrm{~L}$ & 3.568 & $\mathrm{~L}$ & 2.676 & L & 1.784 & $\mathrm{~L}$ & 1.338 & L \\
\hline SA & 27.154 & $\mathrm{~kg}$ & 20.365 & $\mathrm{~kg}$ & 18.103 & $\mathrm{~kg}$ & 13.577 & $\mathrm{~kg}$ & 9.051 & $\mathrm{~kg}$ & 6.788 & $\mathrm{~kg}$ \\
\hline EGDMA & 165.600 & $g$ & 124.200 & $g$ & 110.400 & $g$ & 82.800 & $g$ & 55.200 & $g$ & 41.400 & g \\
\hline AIBN & 273.700 & $g$ & 205.275 & $\bar{g}$ & 182.467 & g & 136.850 & g & 91.233 & $g$ & 68.425 & $g$ \\
\hline Hexane & 690.000 & L & 517.500 & $L$ & 460.000 & $\bar{L}$ & 345.000 & $\bar{L}$ & 230.000 & $\mathrm{~L}$ & 172.500 & L \\
\hline THF & 2300.000 & $\bar{L}$ & 1725.000 & $\mathrm{~L}$ & 1533.333 & $\bar{L}$ & 1150.000 & $\mathrm{~L}$ & 766.667 & $\mathrm{~L}$ & 575.000 & L \\
\hline TCE & 3400.000 & $\bar{L}$ & 2550.000 & $\bar{L}$ & 2278.000 & $\bar{L}$ & 1700.000 & $\bar{L}$ & 1122.000 & $\bar{L}$ & 850.000 & L \\
\hline \multicolumn{13}{|c|}{ NG-18 0.5\% } \\
\hline \multirow[b]{2}{*}{ Chemical } & \multicolumn{12}{|c|}{ Amount needed per fill height } \\
\hline & $100 \%$ & & $75 \%$ & & $67 \%$ & & $50 \%$ & & $33 \%$ & & $25 \%$ & \\
\hline Benzene & 5.730 & L & 4.298 & L & 3.820 & L & 2.865 & $\mathrm{~L}$ & 1.910 & L & 1.433 & L \\
\hline SA & 29.035 & $\mathrm{~kg}$ & 21.776 & $\mathrm{~kg}$ & 19.357 & $\mathrm{~kg}$ & 14.518 & $\mathrm{~kg}$ & 9.678 & $\mathrm{~kg}$ & 7.259 & $\mathrm{~kg}$ \\
\hline EGDMA & 88.780 & $\bar{g}$ & 66.585 & $g$ & 59.187 & $g$ & 44.390 & $g$ & 29.593 & $g$ & 22.195 & $g$ \\
\hline AIBN & 294.400 & $g$ & 220.800 & $g$ & 196.267 & g & 147.200 & $g$ & 98.133 & $g$ & 73.600 & $g$ \\
\hline Hexane & 690.000 & $L$ & 517.500 & $\bar{L}$ & 460.000 & $\bar{L}$ & 345.000 & $\bar{L}$ & 230.000 & $L$ & 172.500 & $\bar{L}$ \\
\hline THF & 2300.000 & $\bar{L}$ & 1725.000 & $\bar{L}$ & 1533.333 & $\bar{L}$ & 1150.000 & $\bar{L}$ & 766.667 & $\bar{L}$ & 575.000 & $\bar{L}$ \\
\hline TCE & 3400.000 & $L$ & 2550.000 & $\bar{L}$ & 2278.000 & $\bar{L}$ & 1700.000 & $\bar{L}$ & 1122.000 & $\mathrm{~L}$ & 850.000 & $\bar{L}$ \\
\hline
\end{tabular}




\subsubsection{Cost comparison}

Table 4. Cost per batch of NG-18 gels compared to trichloroethylene when cleaning tank is filled to various levels

\begin{tabular}{|l|l|l|l|l|}
\hline Cleaning material & NG-18 0.5\% & NG-18 1\% & TCE & $\begin{array}{l}\text { Tank } \\
\text { Level }\end{array}$ \\
\hline & $29,987.16$ & $29,810.55$ & $26,860.45$ & $100.00 \%$ \\
\cline { 2 - 5 } & $22,490.37$ & $22,357.91$ & $20,145.34$ & $75.00 \%$ \\
\cline { 2 - 5 } Cost of Purchase Per Batch (\$) & $19,991.44$ & $19,873.70$ & $17,996.50$ & $66.67 \%$ \\
\cline { 2 - 5 } & $14,993.58$ & $14,905.27$ & $13,430.22$ & $50.00 \%$ \\
\cline { 2 - 5 } & $9,995.72$ & $9,936.85$ & $8,863.95$ & $33.33 \%$ \\
\cline { 2 - 5 } & $7,496.79$ & $7,452.64$ & $6,715.11$ & $25.00 \%$ \\
\hline
\end{tabular}

Table 5. Cost over time of NG-18 gels compared to trichloroethylene when cleaning tank is filled to various levels assuming no depreciation and one use per day.

\begin{tabular}{|c|c|c|c|c|}
\hline Cleaning method & NG-18 0.5\% & NG-18 1\% & TCE & \multirow[b]{2}{*}{ Tank level } \\
\hline Reuse times before cleaning & 3 & 3 & 3 & \\
\hline 1 year cost $(\$)$ & $2,213,784.63$ & $2,211,944.90$ & $2,238,370.76$ & \multirow[t]{3}{*}{$100 \%$} \\
\hline 2 year cost $(\$)$ & $4,427,569.27$ & $4,423,889.79$ & $4,476,741.52$ & \\
\hline 10 year cost $(\$)$ & $22,137,846.33$ & $22,119,448.97$ & $22,383,707.62$ & \\
\hline 1 year cost $(\$)$ & $1,660,338.47$ & $1,658,958.67$ & $1,678,778.07$ & \multirow[t]{3}{*}{$75 \%$} \\
\hline 2 year cost $(\$)$ & $3,320,676.95$ & $3,317,917.34$ & $3,357,556.14$ & \\
\hline 10 year cost $(\$)$ & $16,603,384.75$ & $16,589,586.72$ & $16,787,780.71$ & \\
\hline 1 year cost $(\$)$ & $1,475,856.42$ & $1,474,629.93$ & $1,499,708.41$ & \multirow[t]{3}{*}{$67 \%$} \\
\hline 2 year cost $(\$)$ & $2,951,712.84$ & $2,949,259.86$ & $2,999,416.82$ & \\
\hline 10 year cost $(\$)$ & $14,758,564.22$ & $14,746,299.31$ & $14,997,084.10$ & \\
\hline 1 year cost $(\$)$ & $1,106,892.32$ & $1,105,972.45$ & $1,119,185.38$ & \multirow[t]{3}{*}{$50 \%$} \\
\hline 2 year cost $(\$)$ & $2,213,784.63$ & $2,211,944.90$ & $2,238,370.76$ & \\
\hline 10 year cost $(\$)$ & $11,068,923.16$ & $11,059,724.48$ & $11,191,853.81$ & \\
\hline 1 year cost $(\$)$ & $737,928.21$ & $737,314.97$ & $738,662.35$ & \multirow[t]{3}{*}{$33 \%$} \\
\hline 2 year cost $(\$)$ & $1,475,856.42$ & $1,474,629.93$ & $1,477,324.70$ & \\
\hline 10 year cost $(\$)$ & $7,379,282.11$ & $7,373,149.66$ & $7,386,623.51$ & \\
\hline 1 year cost $(\$)$ & $553,446.16$ & $552,986.22$ & $559,592.69$ & \multirow[t]{3}{*}{$25 \%$} \\
\hline 2 year cost $(\$)$ & $1,106,892.32$ & $1,105,972.45$ & $1,119,185.38$ & \\
\hline 10 year cost $(\$)$ & $5,534,461.58$ & $5,529,862.24$ & $5,595,926.90$ & \\
\hline
\end{tabular}

\subsection{Toxicity}

Both THF and TCE are considered highly toxic in liquid form. THF is not used in liquid form in this experiment. Instead it is used in gel form, which will reduce exposure, and limit the health risks. 


\section{Conclusions and Recommendations}

This study has demonstrated the synthesis and characterization of poly(SA-co-EGDMA) (NG-18) gels. The swelling characteristics of the gels were studied as a function of the solvent polarity and temperature, and the kinetics of swelling were also examined. Volume transition via crystallization of the long alkyl chain was investigated by transmittance at $700 \mathrm{~nm}$ light with controlling temperature. Moreover, the reversibility and reproducibility of the transition were studied by both swelling and transmittance with cyclic temperature change. These properties suggested the utility of NG-18 gels as recyclable VOCs absorbent materials.

The gel system uses THF for swelling, however, cyclohexane or other benign solvents with similar swelling properties may also be used as a swelling agent. In particular cyclohexane is a good possibility with swelling ratio (Q) of 20. Other solvents such as toluene or other non-polar solvents may also be used. No other potential solvents were tested during this phase of the research.

Based on the preliminary cost assessment, the gel cleaning process appears to cost about the same as processes that use alternative cleaners. The gel cleaning process has the advantage of avoiding emissions of hazardous air pollutants (HAPs) and volatile organic compounds (VOCs).

Further research to improve the neutral gel systems with increased swelling properties and recyclability are recommended. Optimization of the gel synthesis, cleaning process and kinetics at room temperature is recommended. 


\section{Acronyms and Abbreviations}

$\begin{array}{ll}\text { Term } & \text { Definition } \\ \text { AIBN } & \text { Azobisisobutyronitrile } \\ \text { ASTM } & \text { American Society for Testing and Materials } \\ \text { CERL } & \text { Construction Engineering Research Laboratory } \\ \text { DC } & \text { Direct Current } \\ \text { DMSO } & \text { Dimethylsulfoxide } \\ \text { DoD } & \text { U.S. Department of Defense } \\ \text { ERDC } & \text { Engineer Research and Development Center } \\ \text { FTIR } & \text { Fourier Transform Infrared } \\ \text { HAP } & \text { Hazardous Air Pollutant } \\ \text { IR } & \text { Infrared } \\ \text { MIBK } & \text { Methylisobutylketone } \\ \text { SA } & \text { Stearylacrylate } \\ \text { SAE } & \text { Society of Automotive Engineers } \\ \text { SERDP } & \text { Strategic Environmental Research and Development Program } \\ \text { SF } & \text { Standard Form } \\ \text { SPOTA } & \text { Sustainable Painting Operations for Total Army } \\ \text { TCE } & \text { Trichloroethylene } \\ \text { TFPB } & \text { Tetraphenylborate } \\ \text { THF } & \text { Tetrahydrofuran } \\ \text { TR } & \text { Technical Report } \\ \text { U.S. } & \text { United States } \\ \text { UV } & \text { Ultraviolet } \\ \text { VOC } & \text { volatile organic compound }\end{array}$




\section{References}

Beebe, D. J . J . S. Moore, J . M. Bauer, Q. Yu, R. H. Liu, C. Devadoss, and B. H. J o. 2000. Functional hydrogel structures for autonomous flow control inside microfluidic channels. Nature. 404:588-590.

Boddu, Veera M. 2011. Oil and Grease Removal Lipophilic Polymer Neutral Gels. Patent disclosure. Submitted to U.S. Army Corps Consul J une 2011.

Gong, J . P., Y. Katsuyama, T. Kurokawa, and Y. Osada. 2003. Double-network hydrogels with extremely high mechanical strength. Adv. Mater. 15:1155-1158.

Iroh, J . O., J . P. Bell, D. A. Scola. 1993. Mechanical-properties of electropolymerized matrix composites. Chem. Mater. 5:78-83.

Ito, K. 2007. Novel cross-linking concept of polymer network: Synthesis, structure, and properties of slide-ring gels with freely movable junctions. Polym. J . 39:489-499.

Kiyonaka, S., K. Sada, I. Yoshimura, S. Shinkai, N. Kato, and I. Hamachi. 2004. Semi-wet peptide/ protein array using supramolecular hydrogel. Nat. Mater. 3:58-64.

Krossing, I., and I. Raabe. 2004. Noncoordinating anions - Fact or fiction? A survey of likely candidates. Angew Chem. Int. Edit. 43:2066-2090.

Laidler, K. J ., J . H. Meiser. 1999. Physical Chemistry, 3d ed. Boston, MA: Houghton Mifflin Company.

MIL-PRF-680B. Degreasing Solvent. 2006

Ohmine, I., and T. Tanaka. 1982. Salt effects on the phase transition of ionic gels. J . Chem. Phys. 77:5725-5729.

Ono, T., S. Shinkai, and K. Sada. 2008. Discontinuous swelling behaviors of lipophilic polyelectrolyte gels in non-polar media. Soft Matter. 4:748-750.

Ono, T., T. Sugimoto, S. Shinkai, and K. Sada. 2007. Lipophilic polyelectrolyte gels as super-absorbent polymers for nonpolar organic solvents. Nat. Mater. 6:429-433.

Ono, T., T. Sugimoto, S. Shinkai, and K. Sada. 2008. Molecular design of super-absorbent polymers for organic solvents by cross-linked lipophilic polyelectrolytes. Adv. Funct. Mater. 18:3936-3940.

Reed, C. A. 1998. Carboranes: A new class of weakly coordinating anions for strong electrophiles, oxidants, and superacids. Acc. Chem. Res. 31:133-139.

Rubinstein, M., R. H. Colby, A. V. Dobrynin, J . F. J oanny. 1996. Elastic modulus and equilibrium swelling of polyelectrolyte gels. Macromolecules 29:398-406. 
Strauss, S. H. 1993. The search for larger and more weakly coordinating anions. Chem. Rev. 93:927-942.

Tanaka, T., and D. J . Fillmore. 1979. Kinetics of swelling of gels. J . Chem. Phys. 70:1214-1218.

Trivedi, H. K., M. L. Massey, R. S. Bhattacharya, G. A. Strahl, and D. Collum. 2004. Cleaners for military machine parts - is there a green alternative? J . Clean. Prod. 12:771-780.

Yoshida, R., K. Uchida, Y. Kaneko, K. Sakai, A. Kikuchi, Y. Sakurai, and T. Okano. 1995. Comb-type grafted hydrogels with rapid de-swelling response to temperaturechanges. Nature 374:240-242. 


\section{Appendix A: Supporting Data for NG-18 Gel Trials}

\section{A.1 NG-18 Gel}

This appendix contains supplemental figures and tables showing the results of the NG-18 gel trials. Figure A-1 shows the metal coupons immersed in gels. Tables A-1 through A-4 list test data and results.

Figure A-1. Metal coupons immersed in gels: (left) NG-18-1\%, (center) NG-18-0.5\%, and (right) toluene.

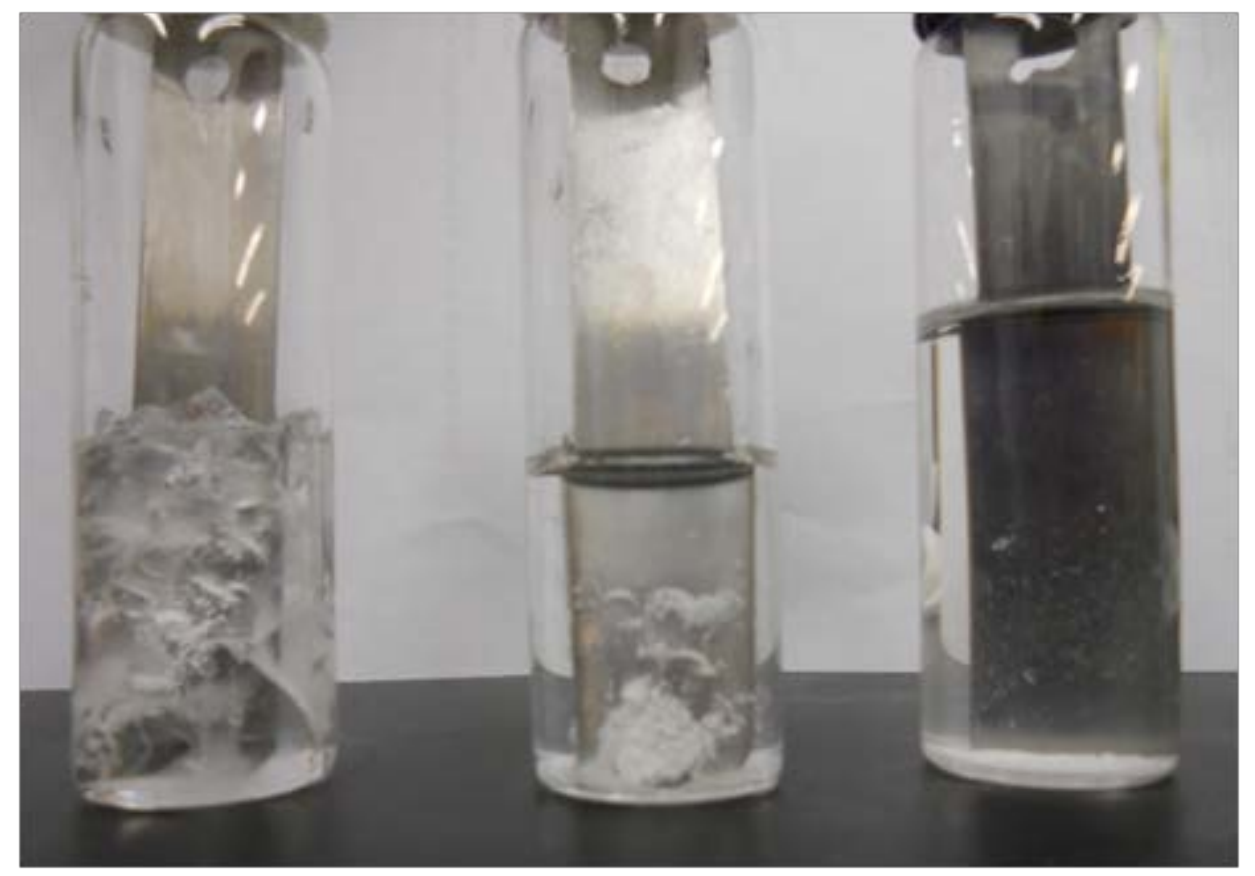


Table A-1. Metal coupon before (b), and after (a) being contaminated with MIL-PRF-10924 grease. Metal coupons cleaned with NG-18 0.5\% (c)-(d) for 13 minutes and 32 seconds, TCE for 5 minutes 12 seconds (e), and NG-18 $1 \%$ for 12 minutes 58 seconds (f).

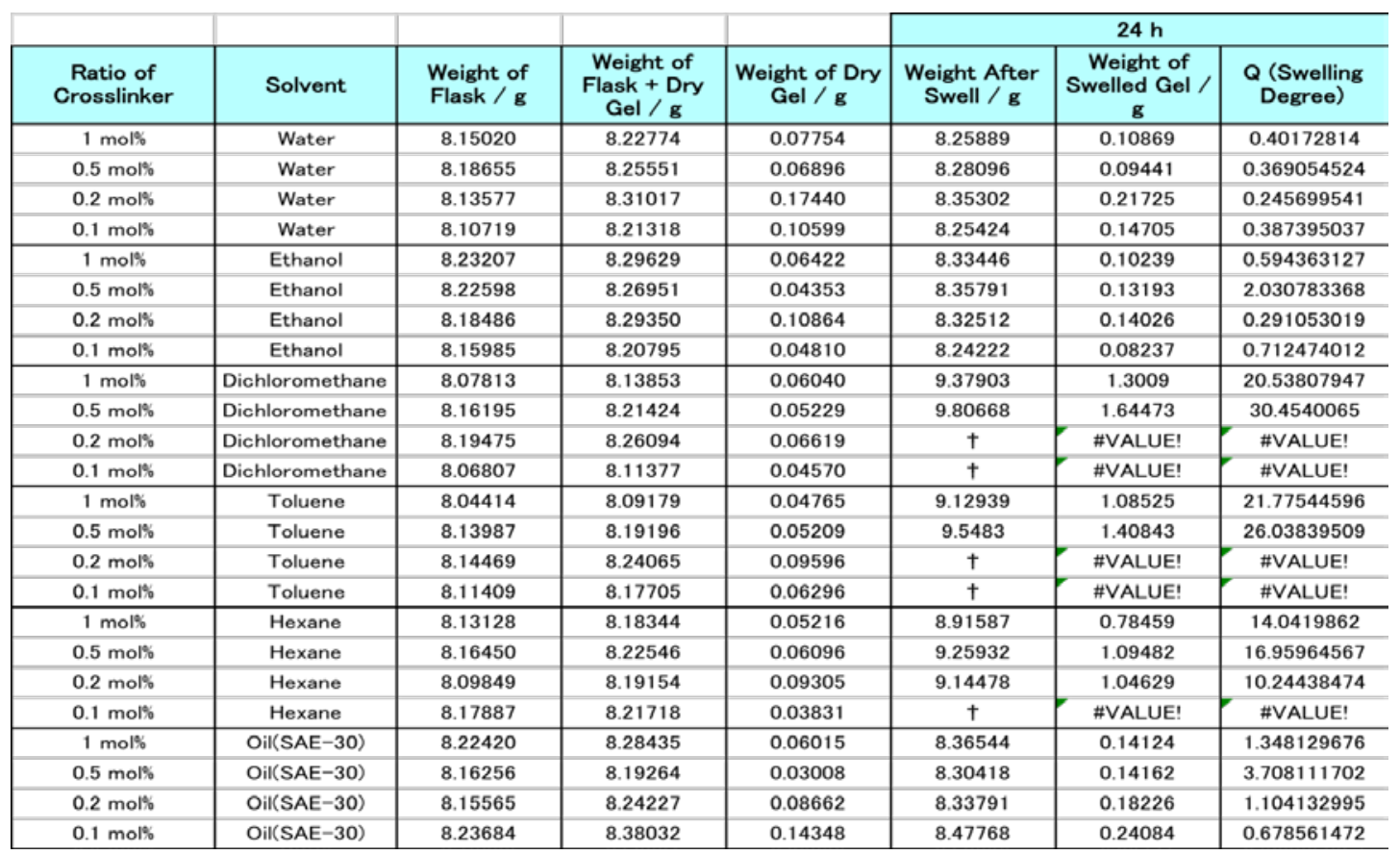

Table A-2. Experimental grease absorption data of NG-18 gels

\begin{tabular}{|l|l|l|l|l|l|l|l|}
\hline $\begin{array}{l}\text { Coupon } \\
\text { Weight }(\mathbf{g})\end{array}$ & $\begin{array}{l}\text { Coupon with } \\
\text { grease } \\
\text { weight }(\mathrm{g})\end{array}$ & $\begin{array}{l}\text { Grease } \\
\text { weight } \\
(\mathbf{g})\end{array}$ & $\begin{array}{l}\text { Cleaning } \\
\text { Product }\end{array}$ & $\begin{array}{l}\text { Time } \\
\text { (min) }\end{array}$ & $\begin{array}{l}\text { Clean } \\
\text { weight (g) } \\
\text { cleaned } \\
(\mathbf{g})\end{array}$ & $\begin{array}{l}\text { Percent of } \\
\text { grease } \\
\text { removed }\end{array}$ \\
\hline 2.65958 & 3.01575 & 0.35617 & NG-18 0.5\% & 13.87 & 2.65847 & 0.35728 & 100.312 \\
\hline 2.67286 & 3.06526 & 0.3924 & NG-18 0.5\% & 13.83 & 2.67409 & 0.39117 & 99.687 \\
\hline 2.73117 & 3.18272 & 0.45155 & NG-18 0.5\% & 13.53 & 2.73248 & 0.45024 & 99.710 \\
\hline 2.57225 & 2.89459 & 0.32234 & NG-18 1\% & 12.97 & 2.57361 & 0.32098 & 99.578 \\
\hline 2.70775 & 3.17597 & 0.46822 & NG-18 1\% & 13.58 & 2.71145 & 0.46452 & 99.210 \\
\hline 2.99139 & 3.37955 & 0.38816 & NG-18 1\% & 13.67 & 2.99347 & 0.38608 & 99.464 \\
\hline 2.42991 & 2.82545 & 0.39554 & TCE & 5.20 & 2.43002 & 0.39543 & 99.972 \\
\hline 2.52677 & 2.97063 & 0.44386 & TCE & 5.17 & 2.52973 & 0.44090 & 99.333 \\
\hline 2.56630 & 2.99553 & 0.42923 & TCE & 5.10 & 2.56653 & 0.42900 & 99.946 \\
\hline
\end{tabular}

Table A-3. Cleaning power data of NG-18 gels.

\begin{tabular}{|l|l|l|l|}
\hline Cleaning Product & Average Percent removed (g) & Average time (min) & Cleaning Power \\
\hline NG-18 1\% & 99.42 & 13.41 & 86.59 \\
\hline NG-18 0.5\% & 99.90 & 13.74 & 86.26 \\
\hline TCE & 99.75 & 5.16 & 94.84 \\
\hline
\end{tabular}


Table A-4. Swelling data of NG-18-x\% gel in various solvents for $24 \mathrm{~h}, 48 \mathrm{~h}$, and $72 \mathrm{~h}$.

\begin{tabular}{|c|c|c|c|c|c|c|c|}
\hline & & & & & \multicolumn{3}{|c|}{$24 \mathrm{~h}$} \\
\hline $\begin{array}{c}\text { Ratio of } \\
\text { Crosslinker }\end{array}$ & Solvent & $\begin{array}{l}\text { Weight of } \\
\text { Flask / g }\end{array}$ & $\begin{array}{l}\text { Weight of } \\
\text { Flask + Dry } \\
\text { Gel / g }\end{array}$ & $\begin{array}{l}\text { Weight of Dry } \\
\text { Gel / g }\end{array}$ & $\begin{array}{l}\text { Weight After } \\
\text { Swell / g }\end{array}$ & $\begin{array}{c}\text { Weight of } \\
\text { Swelled Gel / } \\
\text { g }\end{array}$ & $\begin{array}{c}\text { Q (Swelling } \\
\text { Degree) }\end{array}$ \\
\hline $1 \mathrm{~mol} \%$ & Water & 8.15020 & 8.22774 & 0.07754 & 8.25889 & 0.10869 & 0.40172814 \\
\hline $0.5 \mathrm{~mol} \%$ & Water & 8.18655 & 8.25551 & 0.06896 & 8.28096 & 0.09441 & 0.369054524 \\
\hline $0.2 \mathrm{~mol} \%$ & Water & 8.13577 & 8.31017 & 0.17440 & 8.35302 & 0.21725 & 0.245699541 \\
\hline $0.1 \mathrm{~mol} \%$ & Water & 8.10719 & 8.21318 & 0.10599 & 8.25424 & 0.14705 & 0.387395037 \\
\hline $1 \mathrm{~mol} \%$ & Ethanol & 8.23207 & 8.29629 & 0.06422 & 8.33446 & 0.10239 & 0.594363127 \\
\hline $0.5 \mathrm{~mol} \%$ & Ethanol & 8.22598 & 8.26951 & 0.04353 & 8.35791 & 0.13193 & 2.030783368 \\
\hline $0.2 \mathrm{~mol} \%$ & Ethanol & 8.18486 & 8.29350 & 0.10864 & 8.32512 & 0.14026 & 0.291053019 \\
\hline $0.1 \mathrm{~mol} \%$ & Ethanol & 8.15985 & 8.20795 & 0.04810 & 8.24222 & 0.08237 & 0.712474012 \\
\hline $1 \mathrm{~mol} \%$ & Dichloromethane & 8.07813 & 8.13853 & 0.06040 & 9.37903 & 1.3009 & 20.53807947 \\
\hline $0.5 \mathrm{~mol} \%$ & Dichloromethane & 8.16195 & 8.21424 & 0.05229 & 9.80668 & 1.64473 & 30.4540065 \\
\hline $0.2 \mathrm{~mol} \%$ & Dichloromethane & 8.19475 & 8.26094 & 0.06619 & $\dagger$ & \#VALUE! & \#VALUE! \\
\hline $0.1 \mathrm{~mol} \%$ & Dichloromethane & 8.06807 & 8.11377 & 0.04570 & $\dagger$ & \#VALUE! & \#VALUE! \\
\hline $1 \mathrm{~mol} \%$ & Toluene & 8.04414 & 8.09179 & 0.04765 & 9.12939 & 1.08525 & 21.77544596 \\
\hline $0.5 \mathrm{~mol} \%$ & Toluene & 8.13987 & 8.19196 & 0.05209 & 9.5483 & 1.40843 & 26.03839509 \\
\hline $0.2 \mathrm{~mol} \%$ & Toluene & 8.14469 & 8.24065 & 0.09596 & $\dagger$ & \#VALUE! & \#VALUE! \\
\hline $0.1 \mathrm{~mol} \%$ & Toluene & 8.11409 & 8.17705 & 0.06296 & $\dagger$ & \#VALUE! & \#VALUE! \\
\hline $1 \mathrm{~mol} \%$ & Hexane & 8.13128 & 8.18344 & 0.05216 & 8.91587 & 0.78459 & 14.0419862 \\
\hline $0.5 \mathrm{~mol} \%$ & Hexane & 8.16450 & 8.22546 & 0.06096 & 9.25932 & 1.09482 & 16.95964567 \\
\hline $0.2 \mathrm{~mol} \%$ & Hexane & 8.09849 & 8.19154 & 0.09305 & 9.14478 & 1.04629 & 10.24438474 \\
\hline $0.1 \mathrm{~mol} \%$ & Hexane & 8.17887 & 8.21718 & 0.03831 & $t$ & \#VALUE! & \#VALUE! \\
\hline $1 \mathrm{~mol} \%$ & Oil(SAE-30) & 8.22420 & 8.28435 & 0.06015 & 8.36544 & 0.14124 & 1.348129676 \\
\hline $0.5 \mathrm{~mol} \%$ & Oil(SAE-30) & 8.16256 & 8.19264 & 0.03008 & 8.30418 & 0.14162 & 3.708111702 \\
\hline $0.2 \mathrm{~mol} \%$ & Oil(SAE-30) & 8.15565 & 8.24227 & 0.08662 & 8.33791 & 0.18226 & 1.104132995 \\
\hline $0.1 \mathrm{~mol} \%$ & Oil(SAE-30) & 8.23684 & 8.38032 & 0.14348 & 8.47768 & 0.24084 & 0.678561472 \\
\hline & & \multicolumn{3}{|c|}{$48 \mathrm{~h}$} & \multicolumn{3}{|c|}{$72 \mathrm{~h}$} \\
\hline $\begin{array}{l}\text { Ratio of } \\
\text { Crosslinker }\end{array}$ & Solvent & $\begin{array}{l}\text { Weight After } \\
\text { Swell / g }\end{array}$ & $\begin{array}{c}\text { Weight of } \\
\text { Swelled Gel / } \\
\text { g } \\
\end{array}$ & $\begin{array}{c}\text { Q (Swelling } \\
\text { Degree) }\end{array}$ & $\begin{array}{l}\text { Weight After } \\
\text { Swell / g }\end{array}$ & \begin{tabular}{|c} 
Weight of \\
Swelled Gel / \\
g \\
\end{tabular} & $\begin{array}{c}Q \text { (Swelling } \\
\text { Degree) }\end{array}$ \\
\hline $1 \mathrm{~mol} \%$ & Water & 8.28095 & 0.13075 & 0.686226464 & 8.28699 & 0.13679 & 0.764121744 \\
\hline $0.5 \mathrm{~mol} \%$ & Water & 8.29965 & 0.1131 & 0.640081206 & 8.31614 & 0.12959 & 0.879205336 \\
\hline $0.2 \mathrm{~mol} \%$ & Water & 8.34594 & 0.21017 & 0.205103211 & 8.34243 & 0.20666 & 0.184977064 \\
\hline $0.1 \mathrm{~mol} \%$ & Water & 8.24808 & 0.14089 & 0.329276347 & 8.27606 & 0.16887 & 0.593263515 \\
\hline $1 \mathrm{~mol} \%$ & Ethanol & 8.32767 & 0.0956 & 0.488632825 & 8.33503 & 0.10296 & 0.603238866 \\
\hline $0.5 \mathrm{~mol} \%$ & Ethanol & 8.31440 & 0.08842 & 1.031242821 & 8.30444 & 0.07846 & 0.802435102 \\
\hline $0.2 \mathrm{~mol} \%$ & Ethanol & 8.31820 & 0.13334 & 0.227356406 & 8.33495 & 0.15009 & 0.381535346 \\
\hline $0.1 \mathrm{~mol} \%$ & Ethanol & 8.23548 & 0.07563 & 0.572349272 & 8.24606 & 0.08621 & 0.792307692 \\
\hline $1 \mathrm{~mol} \%$ & Dichloromethane & 9.38906 & 1.31093 & 20.70413907 & 9.32952 & 1.25139 & 19.71837748 \\
\hline $0.5 \mathrm{~mol} \%$ & Dichloromethane & 9.85856 & 1.69661 & 31.44616561 & 9.90836 & 1.74641 & 32.39854657 \\
\hline $0.2 \mathrm{~mol} \%$ & Dichloromethane & + & \#VALUE! & \#VALUE! & $t$ & \#VALUE! & \#VALUE! \\
\hline $0.1 \mathrm{~mol} \%$ & Dichloromethane & $\dagger$ & \#VALUE! & \#VALUE! & $\dagger$ & \#VALUE! & \#VALUE! \\
\hline $1 \mathrm{~mol} \%$ & Toluene & 9.14219 & 1.09805 & 22.04407135 & 9.12388 & 1.07974 & 21.65981112 \\
\hline $0.5 \mathrm{~mol} \%$ & Toluene & 9.95072 & 1.81085 & 33.76387022 & 9.95233 & 1.81246 & 33.79477827 \\
\hline $0.2 \mathrm{~mol} \%$ & Toluene & + & \#VALUE! & \#VALUE! & $t$ & \#VALUE! & \#VALUE! \\
\hline $0.1 \mathrm{~mol} \%$ & Toluene & $\dagger$ & \#VALUE! & \#VALUE! & $\dagger$ & \#VALUE! & \#VALUE! \\
\hline $1 \mathrm{~mol} \%$ & Hexane & 8.92531 & 0.79403 & 14.22296779 & 8.92683 & 0.79555 & 14.2521089 \\
\hline $0.5 \mathrm{~mol} \%$ & Hexane & 9.32132 & 1.15682 & 17.97670604 & 9.24180 & 1.0773 & 16.67224409 \\
\hline $0.2 \mathrm{~mol} \%$ & Hexane & 9.44314 & 1.34465 & 13.45083289 & 9.69641 & 1.59792 & 16.17270285 \\
\hline $0.1 \mathrm{~mol} \%$ & Hexane & $t$ & \#VALUE! & \#VALUE! & $t$ & \#VALUE! & \#VALUE! \\
\hline $1 \mathrm{~mol} \%$ & Oil(SAE-30) & 8.37330 & 0.1491 & 1.478802993 & 8.40869 & 0.18449 & 2.06716542 \\
\hline $0.5 \mathrm{~mol} \%$ & Oil(SAE-30) & 8.30801 & 0.14545 & 3.83543883 & 8.32691 & 0.16435 & 4.463763298 \\
\hline $0.2 \mathrm{~mol} \%$ & Oil(SAE-30) & 8.35000 & 0.19435 & 1.243708151 & 8.36073 & 0.20508 & 1.367582544 \\
\hline $0.1 \mathrm{~mol} \%$ & Oil(SAE-30) & 8.46811 & 0.23127 & 0.61186228 & 8.47708 & 0.24024 & 0.674379704 \\
\hline
\end{tabular}




\section{A.1.1 Materials}

BioAct-MSO Precision Cleaner is manufactured by Petroferm Inc (Figure A-2). The SAE-30 motor oil used here was a Castrol product. The alumina powder, sumicolundum AA-04 was manufactured by Sumitomo Chemical Co. Figure A-3 shows the chemical structures of the BioAct-MSO components.

Figure A-2. A photograph of the containers of (left) BioAct -MSO and (right) SAE-30 oil.

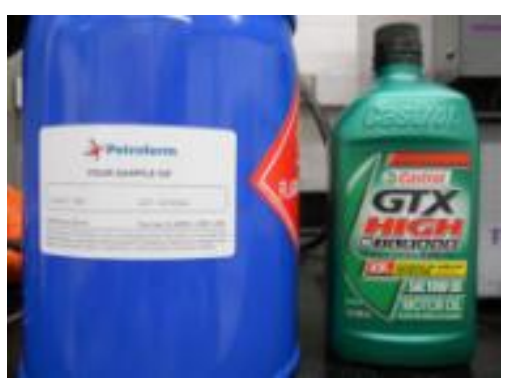

Figure A-3. Chemical structures of BioAct-MSO components: (a) 1-Methyl-4-(1- methylethynyl)cyclohexene (d,I-limonene), (b) sodium dioctylsulfosuccinate, (c) Poly(oxy-1,2-ethanediyl), alpha-(4-nonylphenol)-omega-hydroxy.

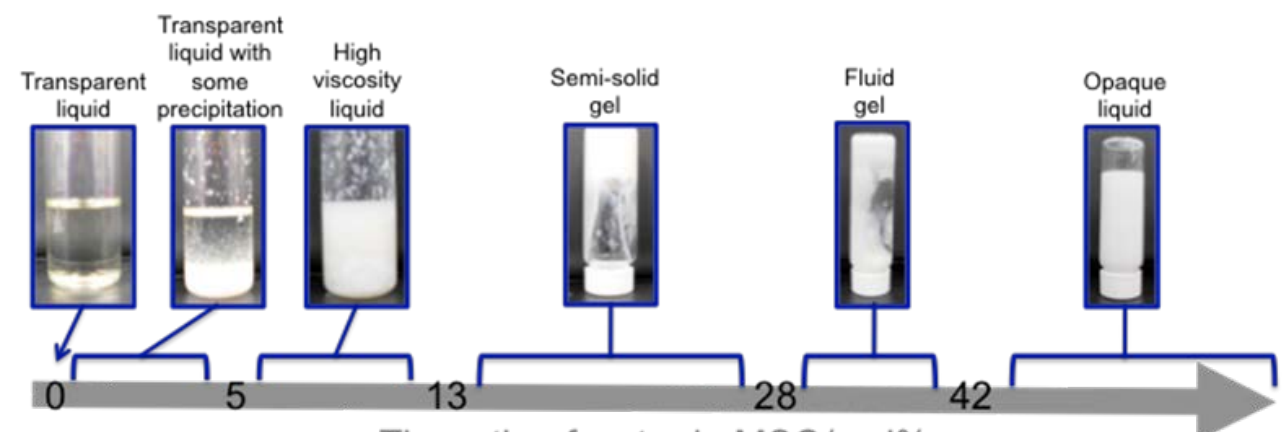

The ratio of water in $\mathrm{MSO} / \mathrm{vol} \%$

\section{A.1.2 Gel formation study}

BioAct-MSO forms a semi-solid gel by mixing 17 - 21 vol\% of water in with the BioAct and shaking vigorously. BioAct MSO will return to liquid form if the mixture is more than $22 \mathrm{vol} \%$ of water. The behaviors were confirmed by adding water to MSO continuously. Six mL of vial tubes were used in this experiment. Figure A-4 shows the result. 
Figure A-4. Sol-gel transition of BioAct-MSO with increasing the water content.

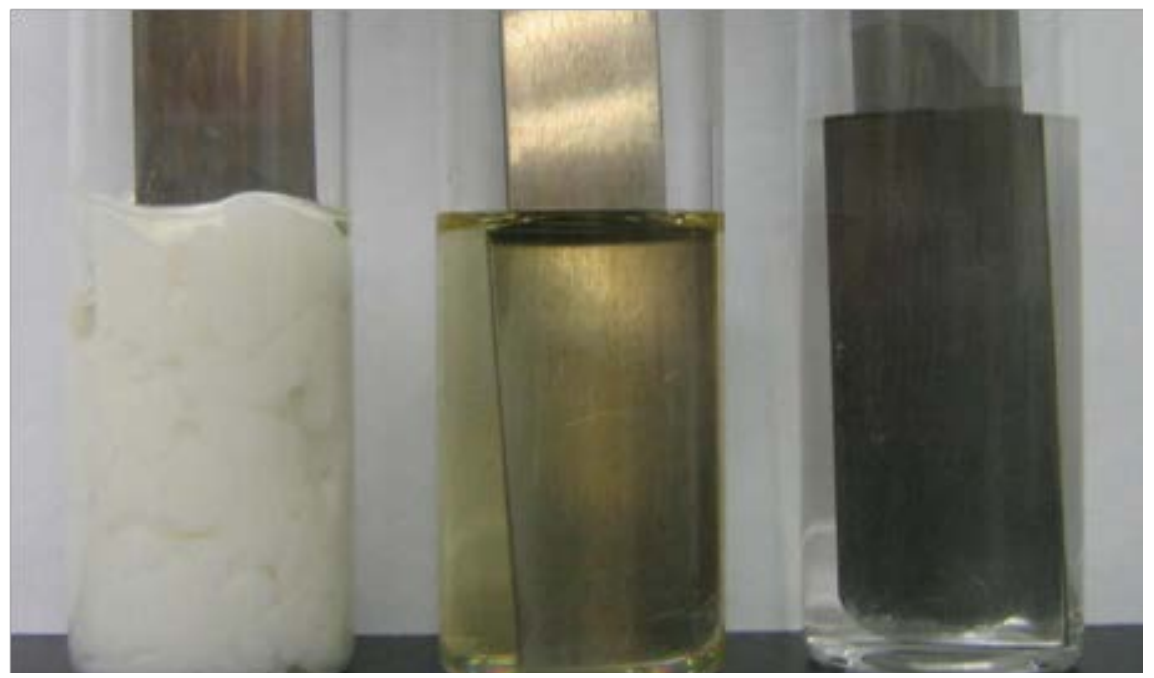

\section{A.1.3 Cleaning studies of soiled metal coupons with BioAct MSO gel}

Stainless steel coupons were washed with acetone and methanol to clean the surface, dried in vacuum for $>72 \mathrm{~h}$, soaked in SAE-30 oil or the mixture of SAE-30 oil and sumicorundum AA-04 alumina powder (= 3/1(w/w)), and placed for 30 min to drop the extra oil (Figure A-5 to A-7). The coupons were then immersed in BioAct-MSO gel or BioAct-MSO only or water for $30 \mathrm{~min}$. BioAct MSO gel was prepared by mixing $82 \mathrm{~mL}$ of BioAct MSO Precision Cleaner with $18 \mathrm{~mL}$ of distilled water vigorously. Oil absorption properties of them were estimated by comparing the coupons' weights between before and after immersion (Figures A-8 to A-10).

Figure A-5. Photographs of coupons soaking in (left) BioAct-MSO gel, (center) BioAct-MSO only, and (right) water.

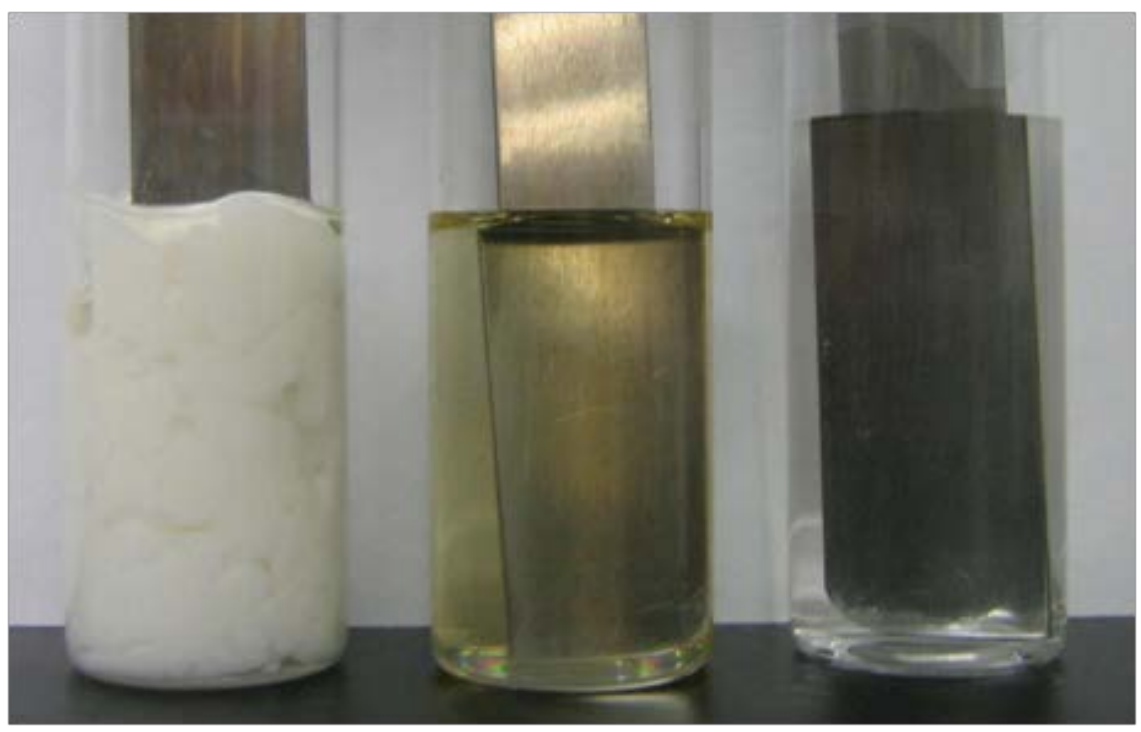


Figure A-6. Photographs of metal coupon (a) coated with oil (b)-(d) after immersion: (b) BioAct-MSO gel, (c) BioAct-MSO only, and (d) water.
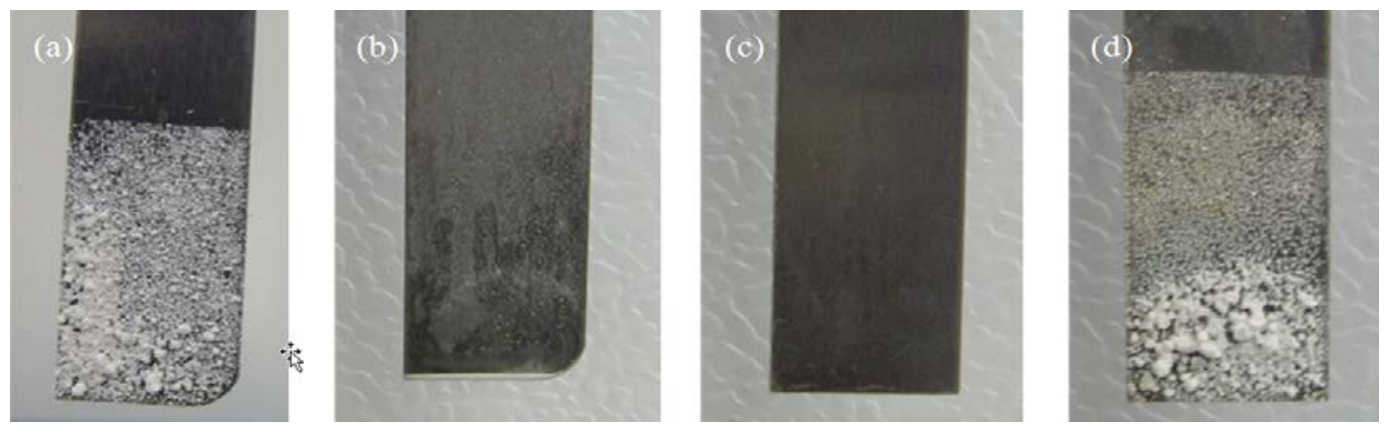

Figure A-7. Photographs of metal coupons (a) coated with oil and alumina powder, (b)-(d) after immersion: (b) BioAct-MSO gel, (c) BioAct-MSO, and (d) water.
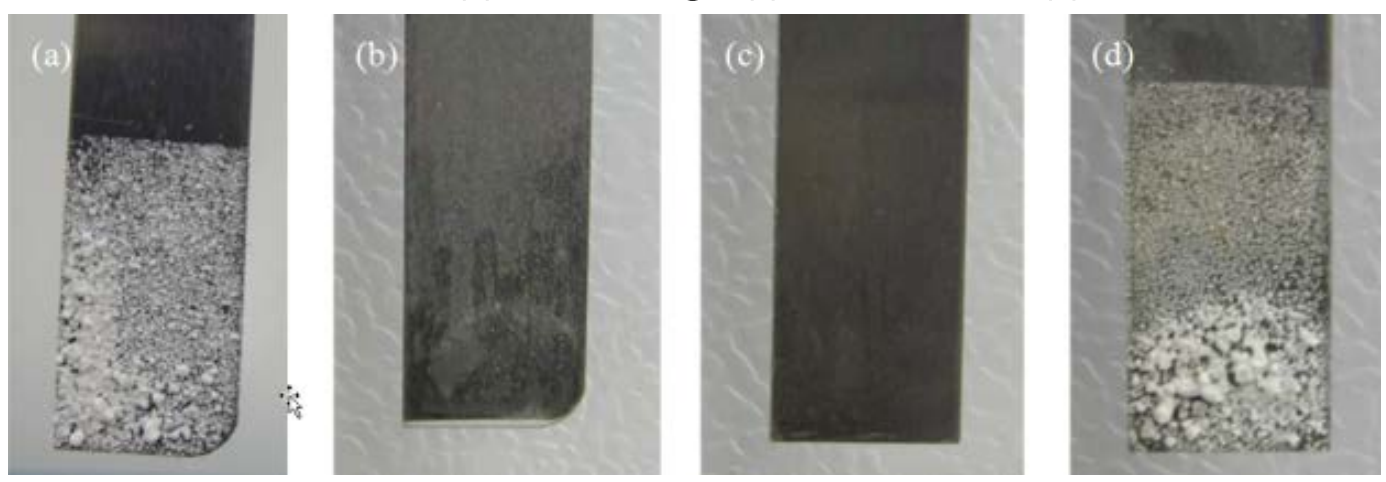

Figure A-8. Cleaning properties of BIOACT-MSO.

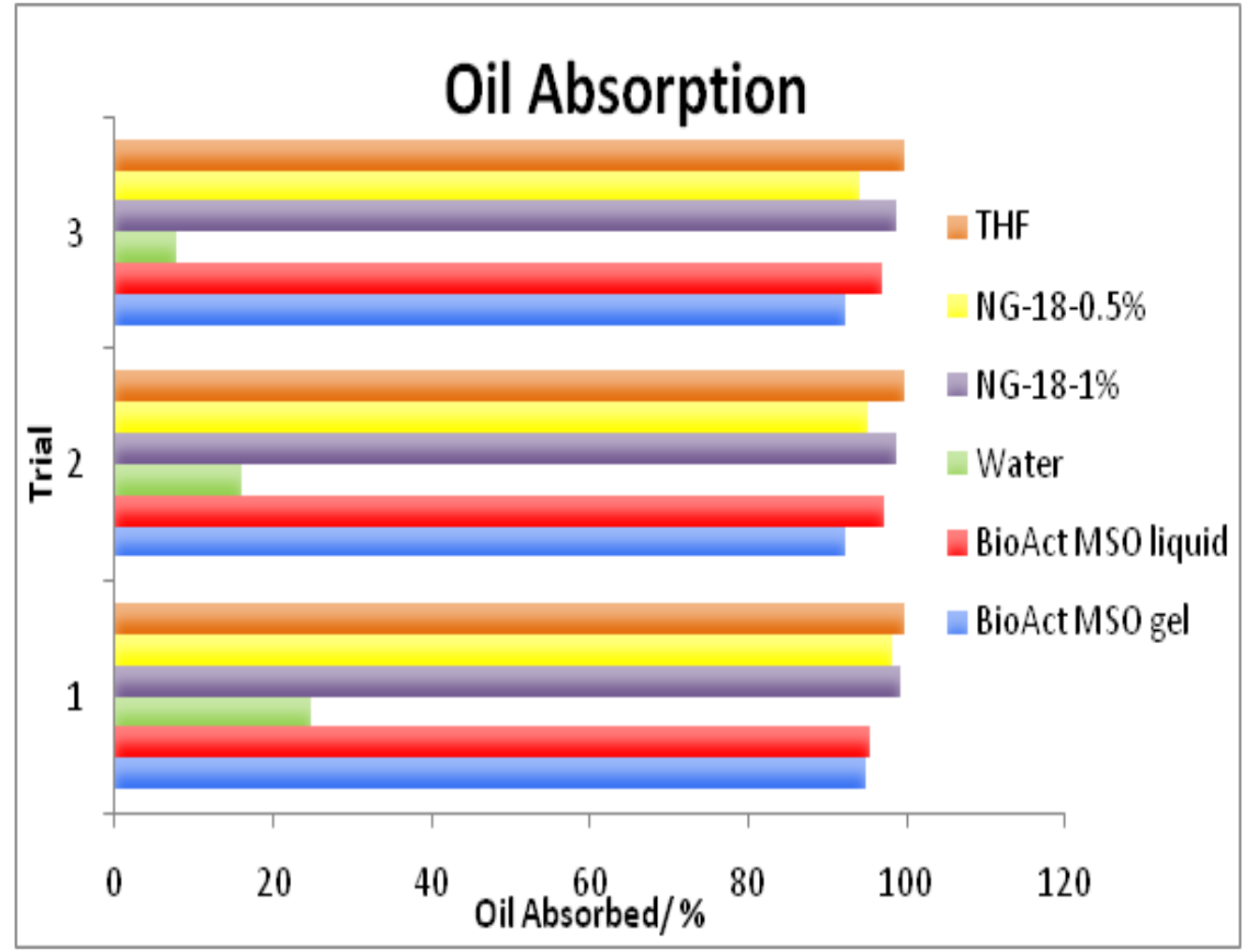


Figure A-9. Oil absorption properties of BioAct MSO compared to NG-18, water and THF.

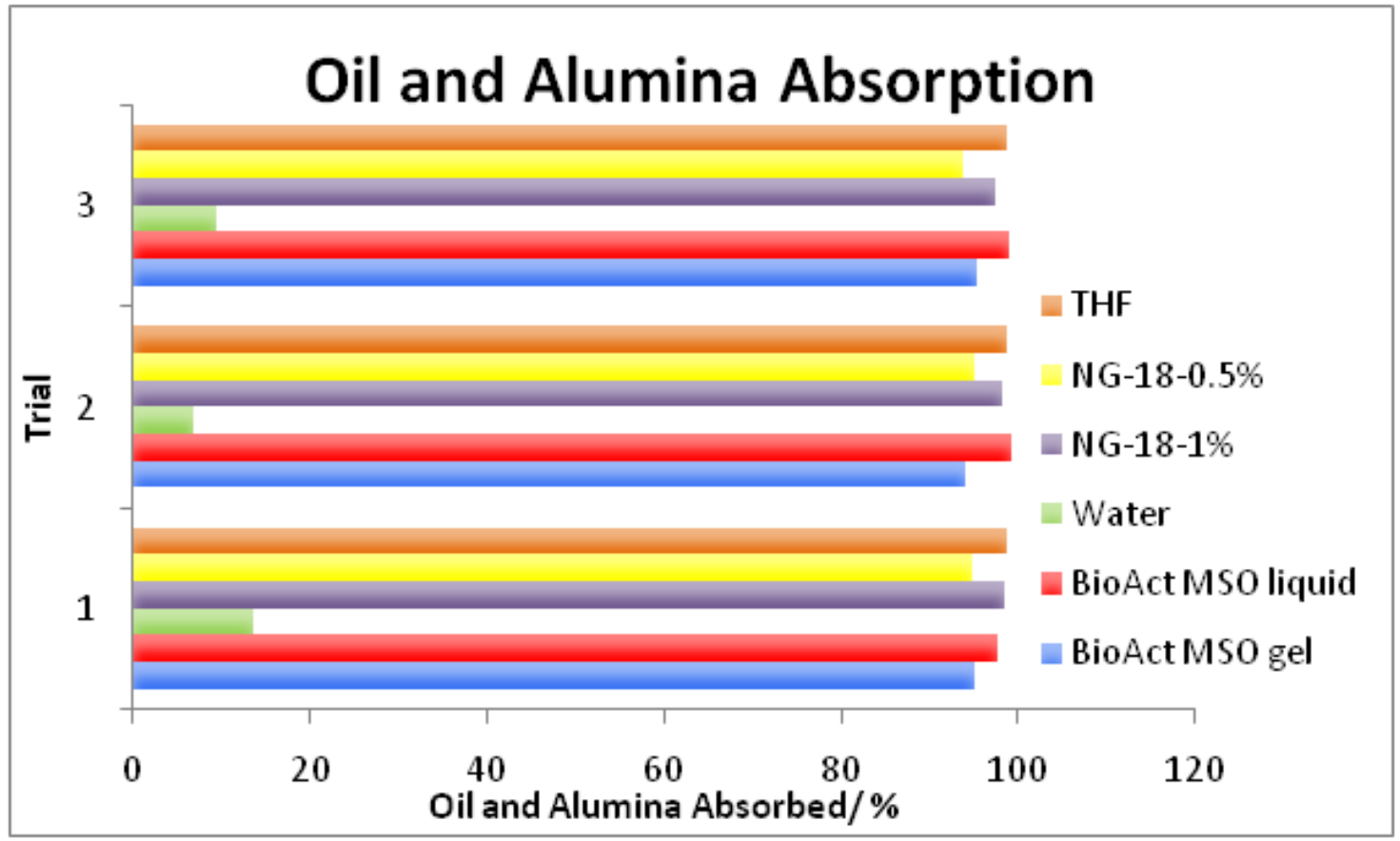

Figure A-10. Oil and alumina absorption properties of BioAct MSO and NG-18 gels compared to THF and water.

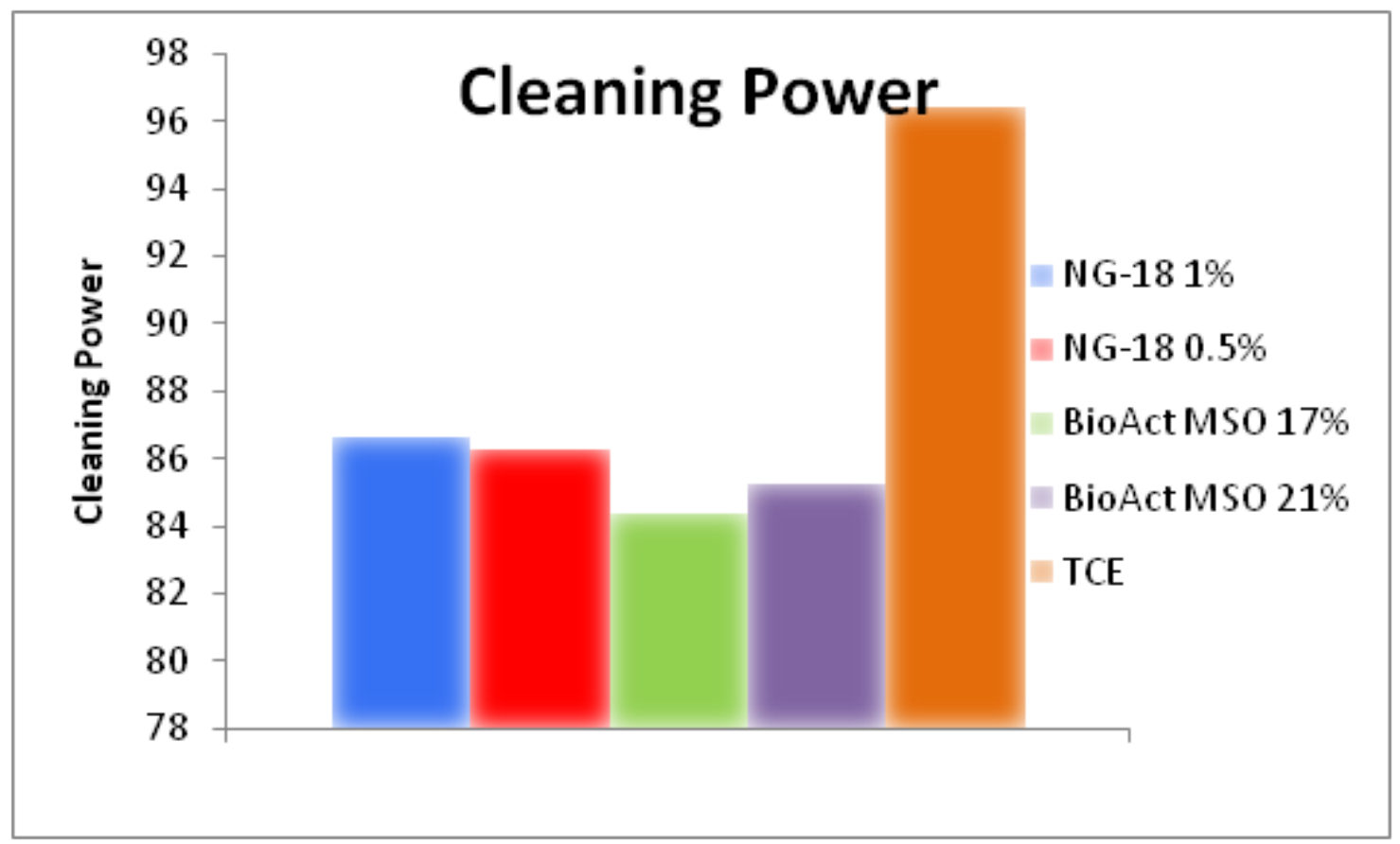

Compared to NG-18 gels, the cleaning properties were not as good. This is attributed to the tendency of BioAct- MSO gels to stay on the metal surface. These residual gels were able to be removed by soaking in water again. In the case of coupons with oil and alumina powder washed by 
BioAct MSO gel (Figure A-7), some alumina powder stayed on the surface. They could be cleaned by rubbing them in water. This is attributed to the fact that BioAct MSO emulsion gel is softer and does not tighten on the coupon as much as polymer matrix gels such as NG-18. BioAct has slightly greater grease absorption than the NG-18 gel, but had approximately the same cleaning power (Figures A-11 and A-12).

Figure A-11. Cleaning Power of BioAct MSO and NG-18 gels compared to trichloroethylene.

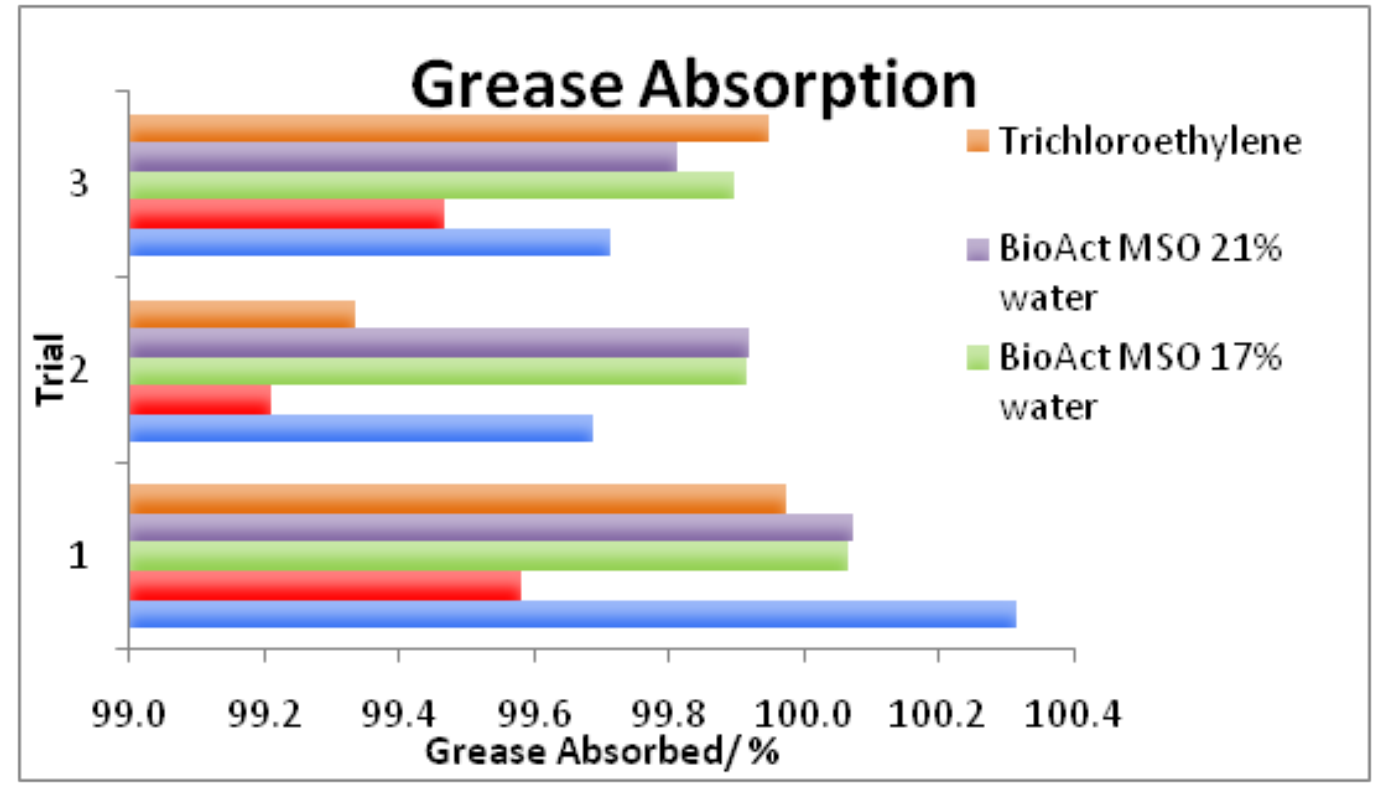

Figure A-12. Grease absorption properties of BioAct MSO and NG-18 gels compared to trichloroethylene.

(a)

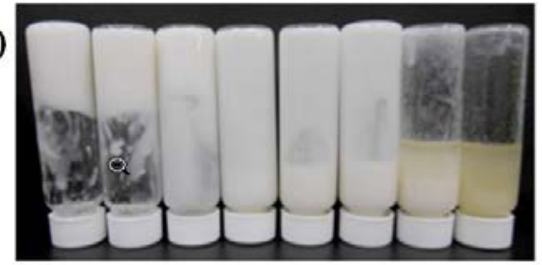

(b)

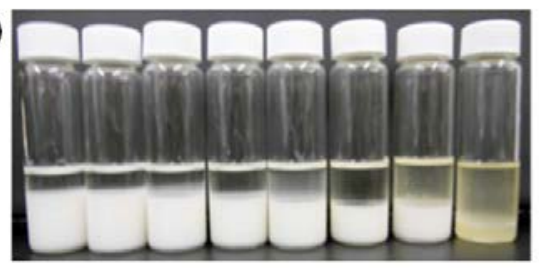

(c)

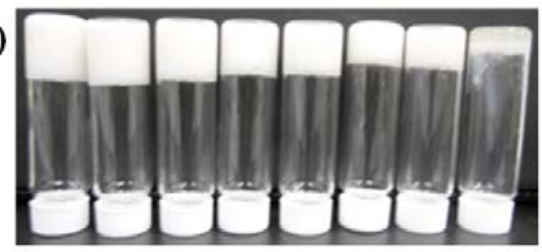

(d)

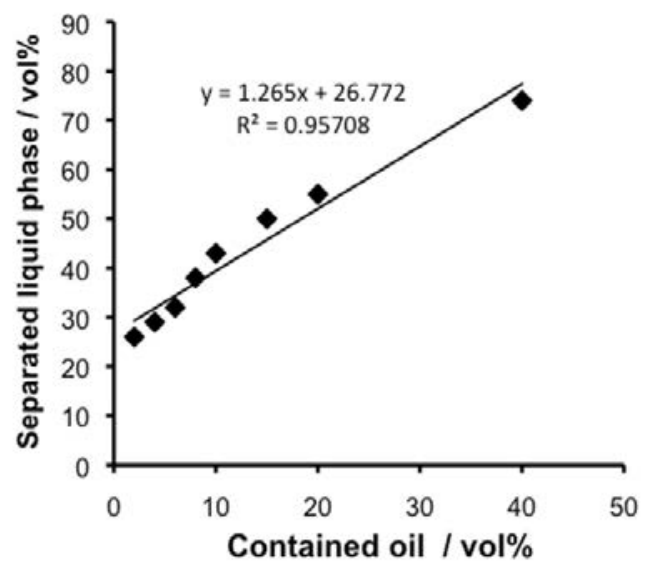




\section{A.1.4 Construction of the recycling system}

\section{A.1.4.1 Trial 1: Heating at $60{ }^{\circ} \mathrm{C}$}

Different ratios of oil were added to BioAct-MSO gel (BioAct-

$\mathrm{MSO} /$ water $=82 / 18(\mathrm{v} / \mathrm{v})): 2,4,6,8,10,15,20$, and $40 \mathrm{vol} \%$ for the total volume. After mixing them vigorously, they were heated at $60{ }^{\circ} \mathrm{C}$ for 30 min and separated into two phases. Cooling to room temperature caused the gelation of the lower phase, thus each phase was denoted as liquid and gel phase. The amount of liquid phase is shown in Figure A-13 (d), and each phase was measured by IR spectroscopy to investigate the ratio of oil content (Figures A-14 to A-17).

Figure A-13. (a)-(c) Photographs of BIOACT-MSO+oil: from left side, 2, 4, 6, 8, 10, 15, 20, and $40 \mathrm{vol} \%$ of oil was contained. (a) before heated, (b) cooled to r.t. after heated to $60^{\circ} \mathrm{C}$, and

(c) separated gel phase after heated. (d) The changes of the amount of liquid phase after heated.

(a)

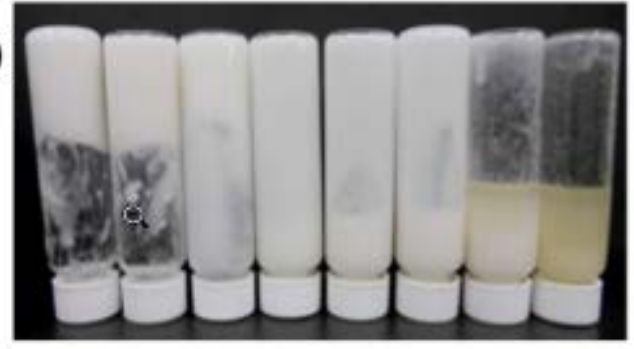

(b)

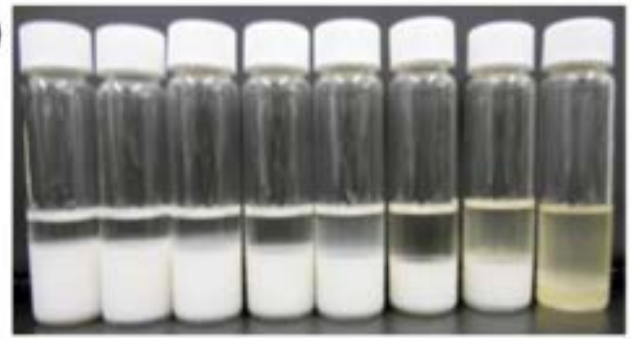

(c)

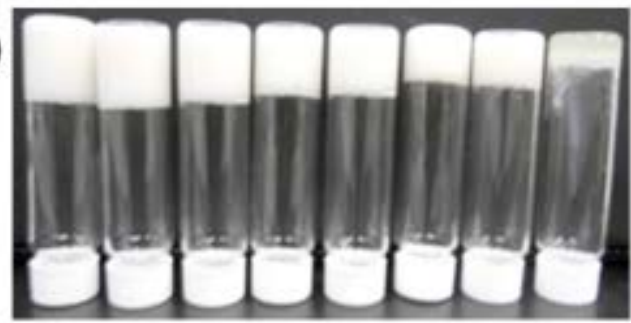

(d)

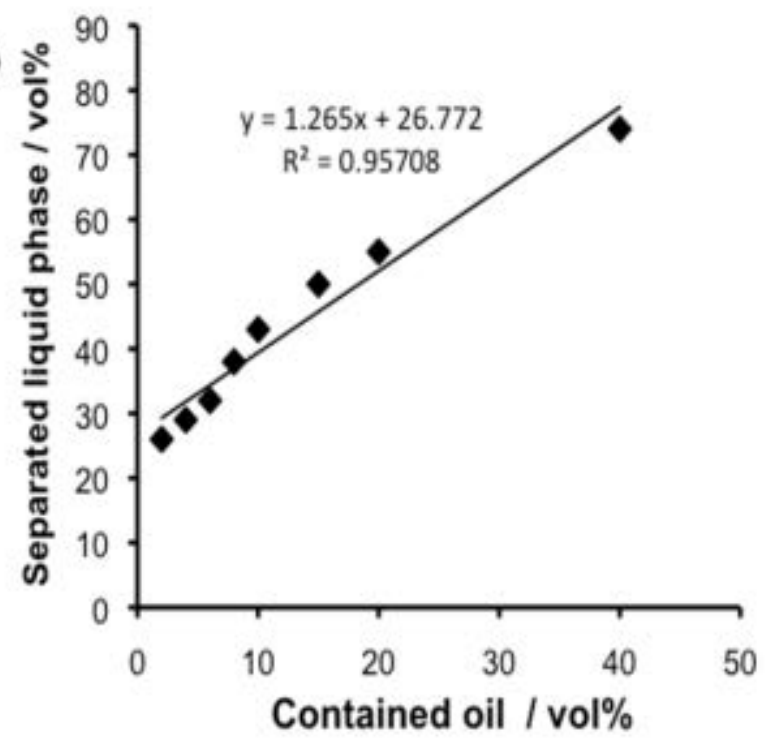


Figure A-14. IR spectra of BioAct MSO, SAE-30 oil, and their mixtures. $1400-1500 \mathrm{~cm}-1$ region was magnified to investigate the ratio of oil content in liquid and gel.

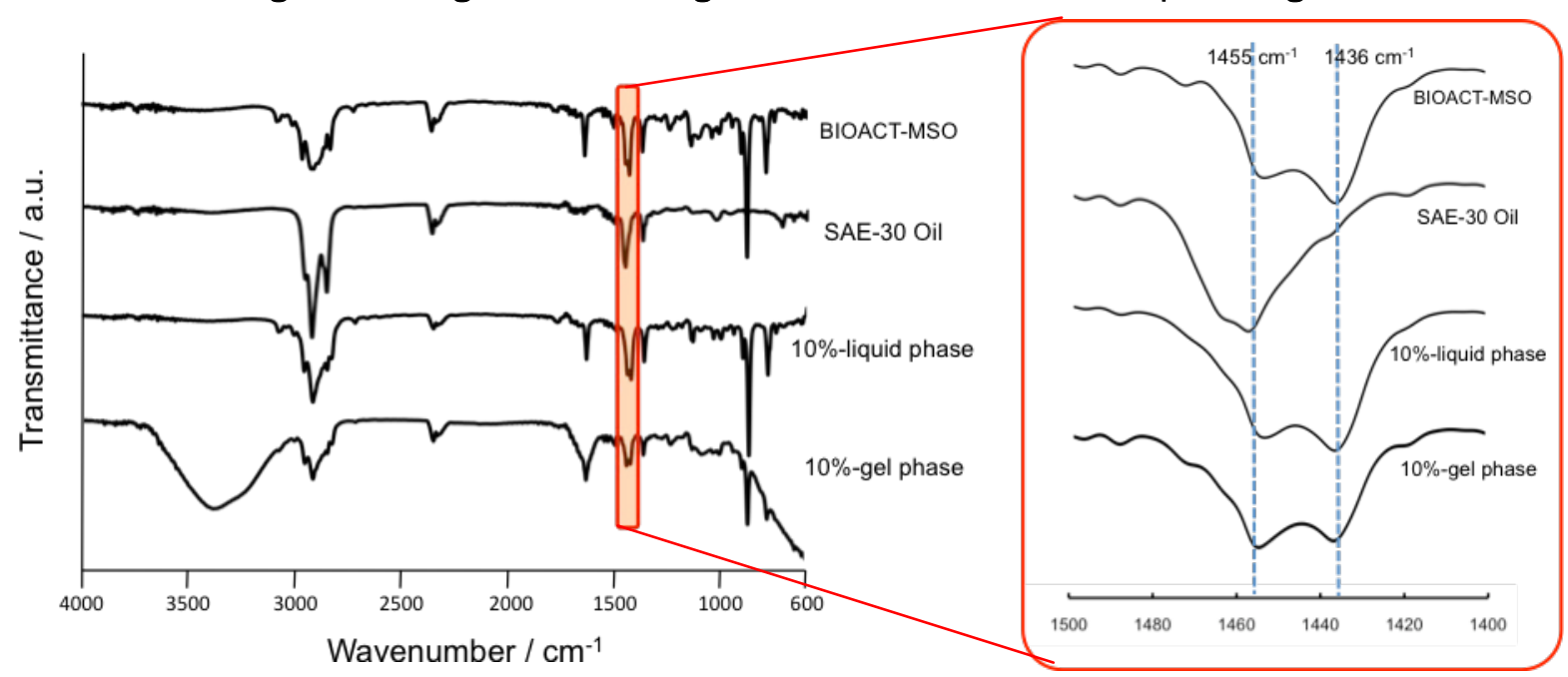

Figure A-15. The changes of $1455 \mathrm{~cm}-1$ with increment of oil content when the spectra were normalized at $1436 \mathrm{~cm}-1$ : (a) liquid phase, (b) gel phase.

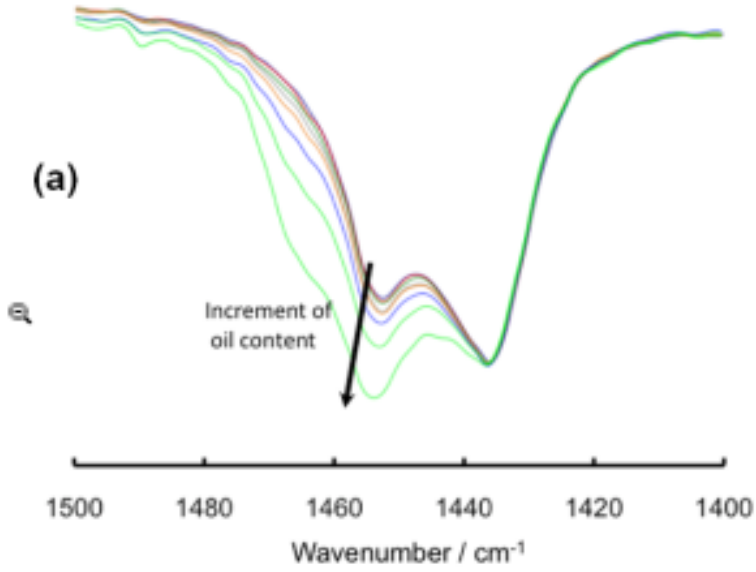

(b)
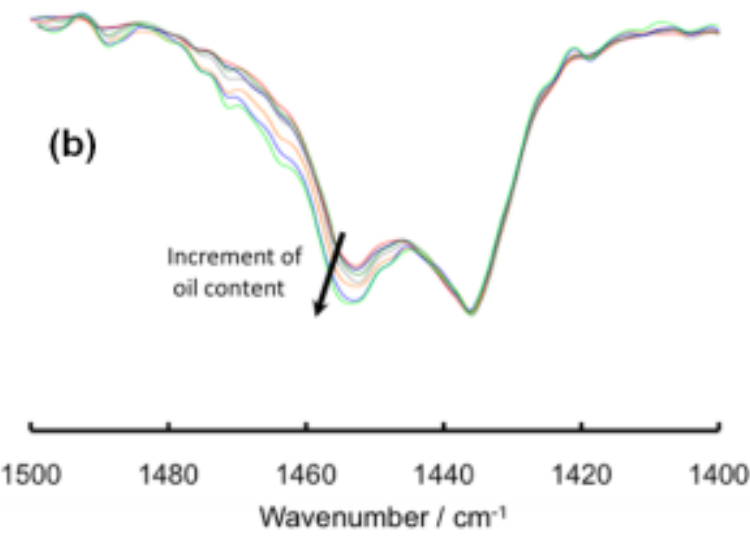

The quantification of oil content in each phase was investigated by comparing the intensities of peaks at 1455 and $1436 \mathrm{~cm}-1$. These peaks originated from carbonyl vibration. When BioAct and oil were mixed, the intensity was presented as the addition of them: $e=a+c, f=b+d$ (Figure A-16). Thus, the oil content of each phase $(\theta)$ is indicated as:

$$
\theta=\frac{\text { af }- \text { be }}{\text { af }- \text { be }-c f+\text { de }}
$$


Figure A-16. The intensities of 1455 and $1426 \mathrm{~cm}-1$ in each spectrum were indicated as a-f.

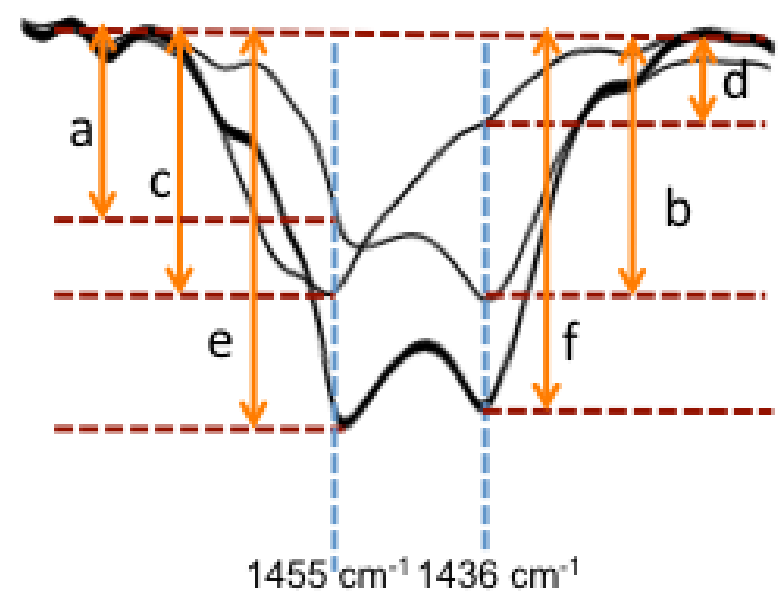

Figure A-17 shows the calculated results.

Figure A-17. The oil content of each phase calculated from intensities of IR spectra: (left) liquid phase, (right) gel phase.
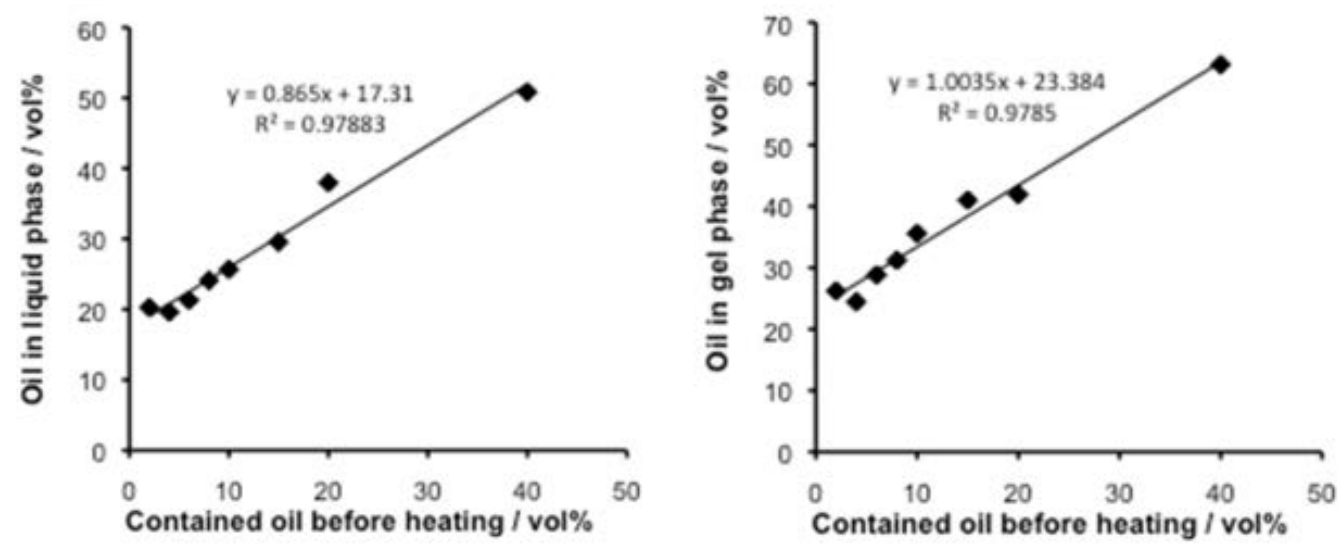

\section{A.1.4.2 Trial 2}

Five $\mathrm{mL}$ of the oil was mixed with $90 \mathrm{~mL}$ of MSO gels (BioAct$\mathrm{MSO} /$ water $=82 / 18(\mathrm{v} / \mathrm{v})$ ). After stirring vigorously, the gel was diluted by adding $95 \mathrm{~mL}$ of water. The homogenous suspension liquid was filtrated with three types of filters in Table A-5. The compression pressure is $60 \mathrm{psi}$. The filtrate and residue were measured by IR spectroscopy to investigate the components. 
Table A-5. The list of filter used in this experiment.

\begin{tabular}{|l|l|l|c|c|}
\hline Manufacturer & Name & Product No. & Pore Size & Material \\
\hline Sterlitech & TF UF GH & YMGHSP3001 & $0.1 \mu \mathrm{m}$ & - \\
\hline Sterlitech & TF UF GK & YMGHSP3001 & $0.1 \mu \mathrm{m}$ & - \\
\hline Fisher & Qualitative P2 & 09-803-5F & $1-5 \mu \mathrm{m}$ & Cellulose \\
\hline
\end{tabular}

Figure A-18. (a) Photographs of the filtrate by using different pore size filter: from left side, $0.1,0.2$, and 1-5 $\mu \mathrm{m}$. (b) A photograph of the filtration apparatus. (c) IR spectra of each components, residue, and filtrate.

(a)

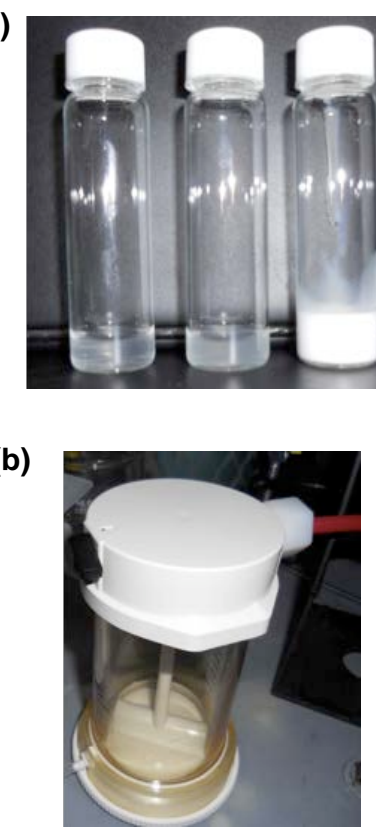

(c)

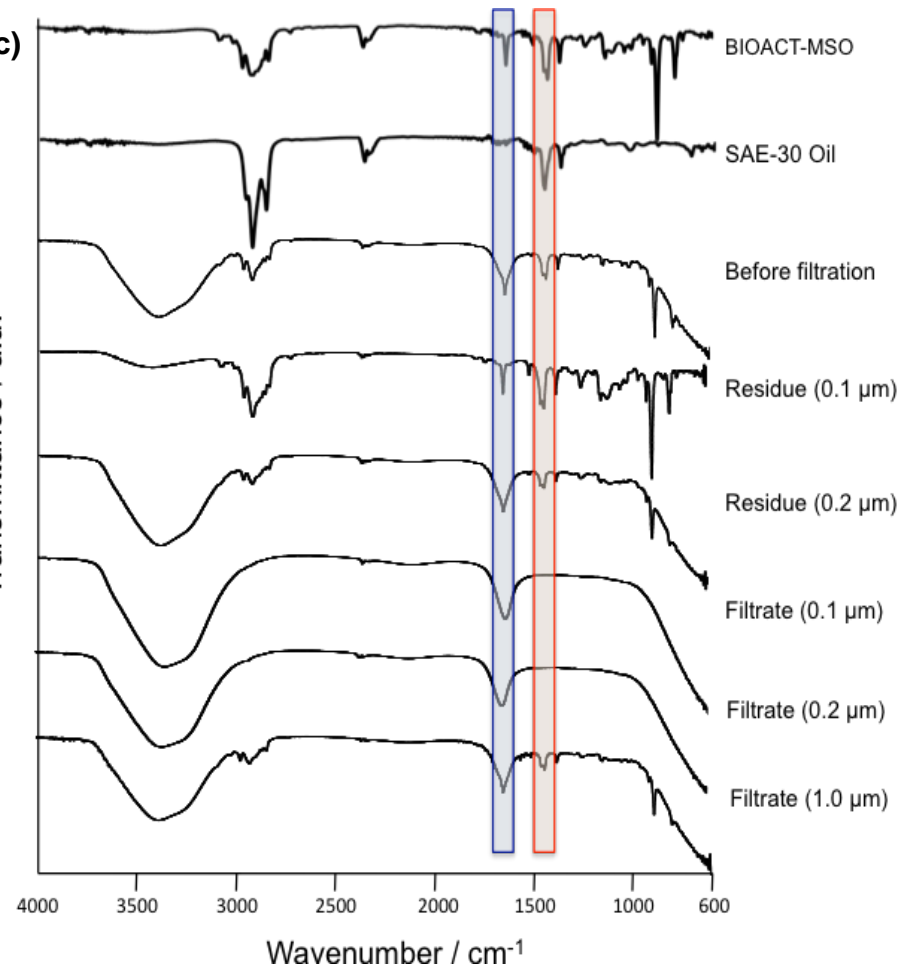

In

Figure A-18(c), the blue region originated from $\mathrm{C}=\mathrm{C}$ vibration of limonene, and the red region showed carbonyl vibration of surfactant and oil. With the filters of pore size $<0.2 \mu \mathrm{m}$, limonene and water were separated from the suspension The filtrate was transparent and had lemon scent. However, the residues include oil, limonene, and surfactant, and they cannot be separated in this method.

\section{A.2 Conclusion}

BioAct-MSO gels have good cleaning properties for soiled metal surface (Figures A-19 to A-22, Tables A-6 and A-7). However, it was not possible to construct the recycling system. BioAct MSO is the mixture of some sur- 
factants and limonene, so the oil not only mixes with limonene, but also is confined in micelle. To extract oil in this mixture, it is necessary to combine filtration, adsorption, and some other method.

Table A-6. Experimental grease absorption data of NG-18 gels.

\begin{tabular}{|l|l|l|l|l|l|l|l|}
\hline $\begin{array}{l}\text { Coupon } \\
\begin{array}{l}\text { Weight } \\
\text { (g) }\end{array}\end{array}$ & $\begin{array}{l}\text { Coupon } \\
\text { with } \\
\text { grease } \\
\text { weight } \\
\text { (g) }\end{array}$ & $\begin{array}{l}\text { Grease } \\
\text { weight } \\
\text { (g) }\end{array}$ & Cleaning Product & $\begin{array}{l}\text { Time } \\
(\mathrm{min})\end{array}$ & $\begin{array}{l}\text { Clean } \\
\text { weight } \\
(\mathrm{g})\end{array}$ & $\begin{array}{l}\text { Amount } \\
\text { cleaned } \\
\text { (g) }\end{array}$ & $\begin{array}{l}\text { Percent of } \\
\text { grease } \\
\text { removed }\end{array}$ \\
\hline 2.90688 & 3.30909 & 0.40221 & BioAct MSO 17\% & 15.38 & 2.90663 & 0.40246 & 100.062 \\
\hline 2.60016 & 3.00146 & 0.40130 & BioAct MSO 17\% & 17.41 & 2.60051 & 0.40095 & 99.913 \\
\hline 2.85997 & 3.25160 & 0.39163 & BioAct MSO 17\% & 14.17 & 2.86038 & 0.39122 & 99.895 \\
\hline 2.90204 & 3.29699 & 0.39495 & BioAct MSO 21\% & 13.57 & 2.90176 & 0.39523 & 100.071 \\
\hline 2.58714 & 2.96342 & 0.37628 & BioAct MSO 21\% & 13.70 & 2.58745 & 0.37597 & 99.918 \\
\hline 2.44264 & 2.85091 & 0.40827 & BioAct MSO 21\% & 17.08 & 2.44342 & 0.40749 & 99.809 \\
\hline 2.72486 & 3.15149 & 0.42663 & TCE & 1.97 & 2.72484 & 0.42665 & 100.005 \\
\hline 2.82179 & 3.23500 & 0.41321 & TCE & 2.20 & 2.82172 & 0.41328 & 100.017 \\
\hline 2.70015 & 3.12888 & 0.42873 & TCE & 1.80 & 2.70008 & 0.42880 & 100.016 \\
\hline
\end{tabular}

Table A-7. Cleaning power data of BioAct MSO gels.

\begin{tabular}{|l|c|c|c|}
\hline Cleaning Product & $\begin{array}{l}\text { Average Percent } \\
\text { removed }(\mathrm{g})\end{array}$ & $\begin{array}{l}\text { Average time } \\
(\mathrm{min})\end{array}$ & Cleaning Power \\
\hline BioAct MSO 17\% & 99.957 & 15.65 & 84.35 \\
\hline BioAct MSO 21\% & 99.932 & 14.78 & 85.22 \\
\hline TCE & 100.013 & 1.99 & 98.01 \\
\hline
\end{tabular}

Figure A-19. Metal coupon (a) coated with oil, and after immersion in: (b) BioAct MSO gel, (c) BioAct MSO, and (d) water.
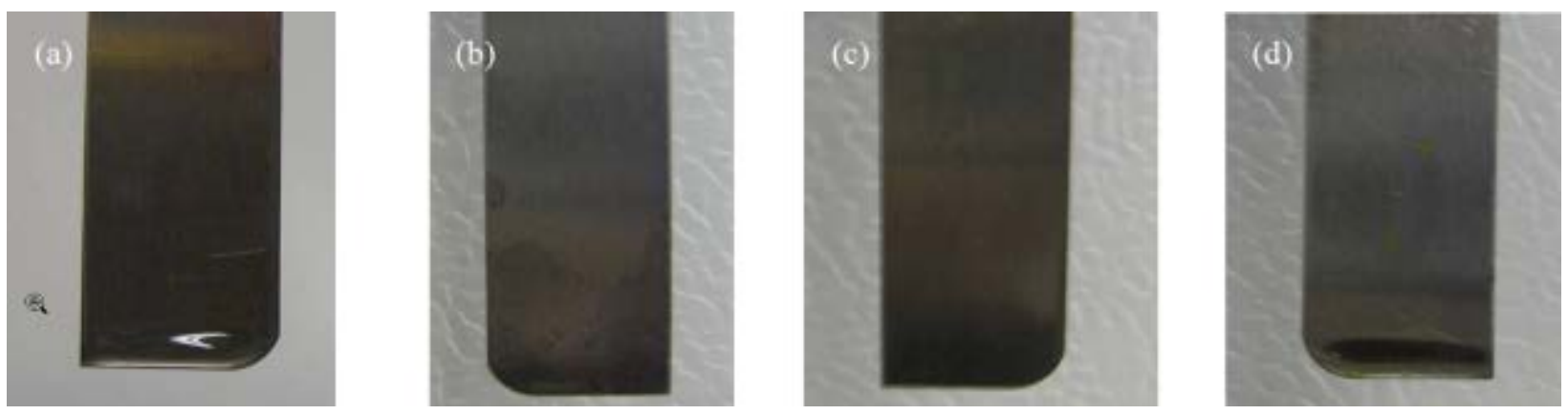
Figure A-20. Coupons soaking in (left) BioAct MSO gel, (center) BioAct MSO only, and (right) water.

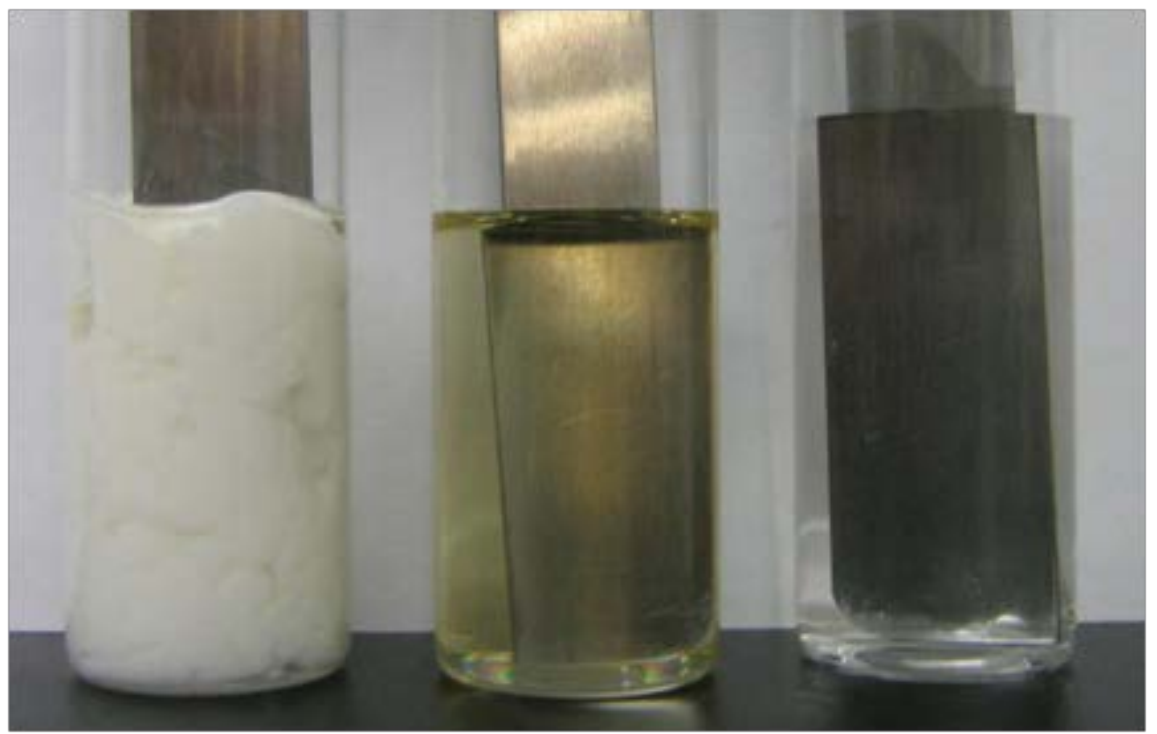

Figure A-21. Metal coupons (a) coated with oil and alumina powder, and after immersion in: (b) BioAct MSO gel, (c) BioAct MSO, and (d) water.

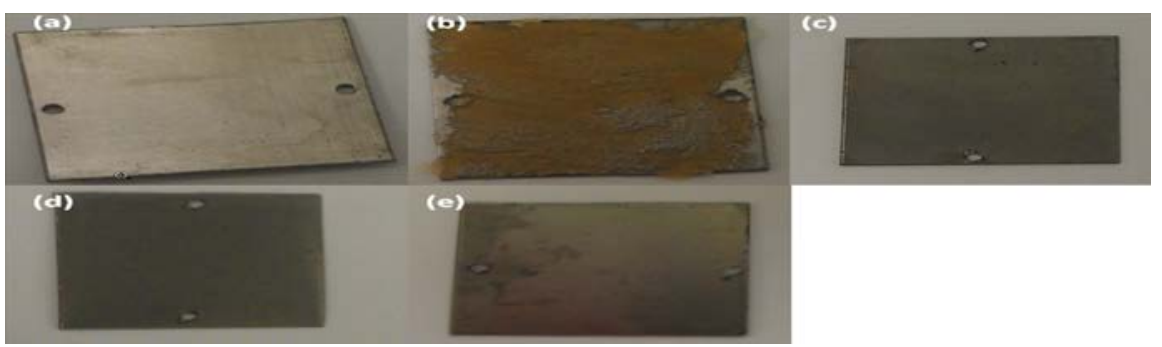

Figure A-22. Uncontaminated metal coupon (a), contaminated metal coupon (b), and coupons cleaned with: TCE (c), and BioAct MSO with $17 \%$ water (d) and $21 \%$ water (e).

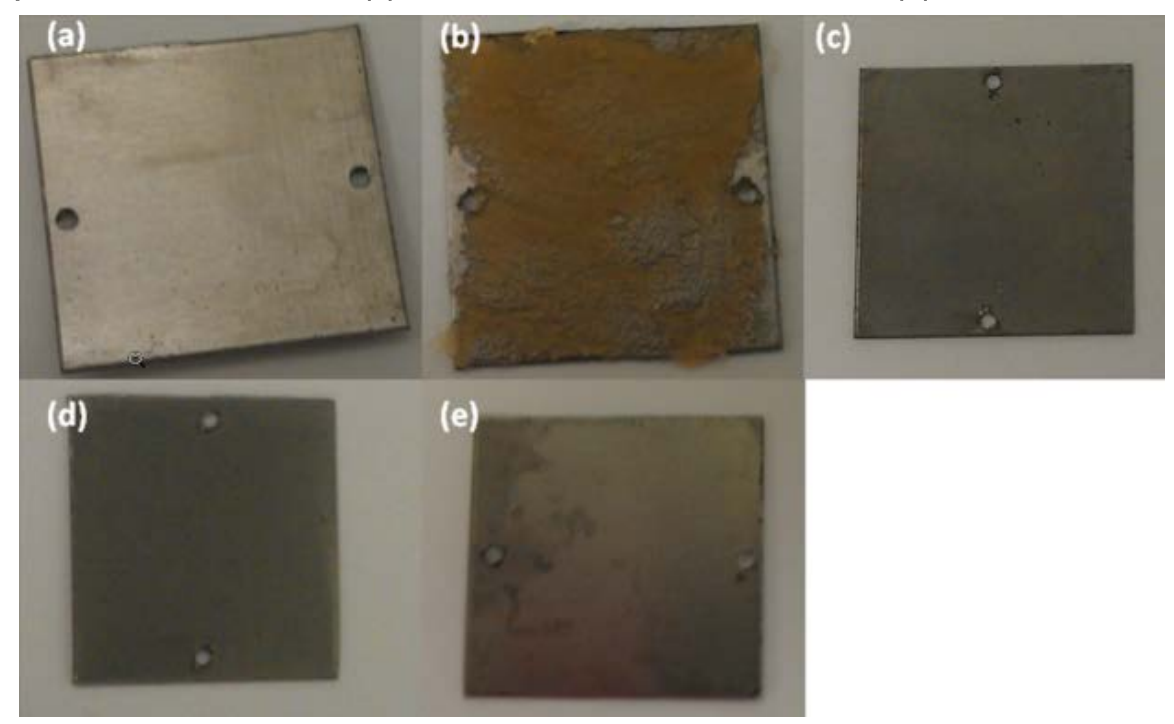




\section{Appendix B: Material Safety Data Sheets Imbiber beads, TCE, and THF}

SIGMA-ALDRICH

sigma-aldrich.com

Material Safety Data Sheet

Version 4.1

Revision Date 02/13/201

Print Date 05/24/2011

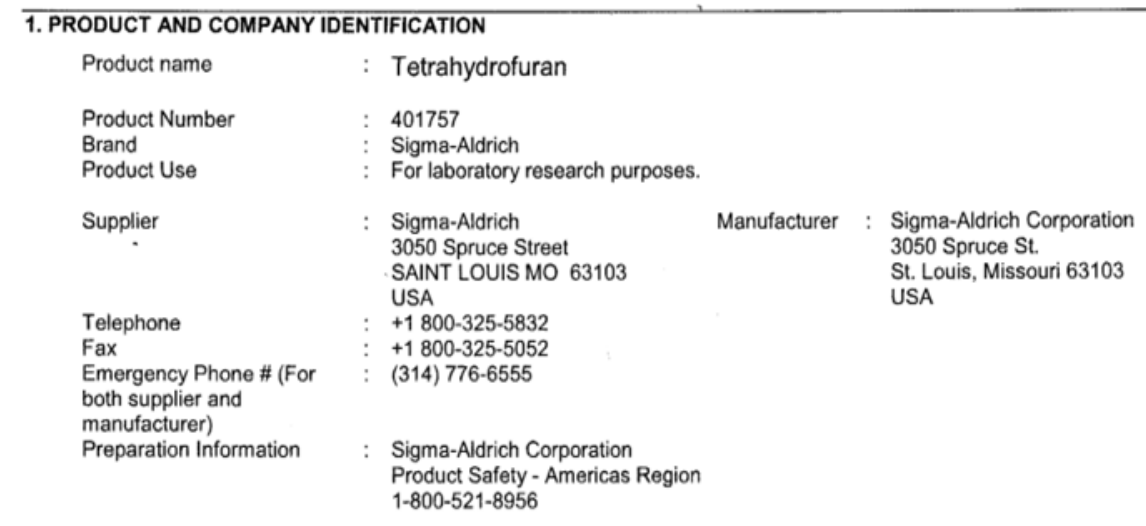

2. HAZARDS IDENTIFICATION

Emergency Overview

OSHA Hazards

Flammable liquid, Target Organ Effect, Harmful by ingestion., Irritant, Carcinogen

Target Organs

Central nervous system, Liver, Kidney

Other hazards which do not result in classification

May form explosive peroxides.

GHS Classification

Flammable liquids (Category 2)

Acute toxicity, Oral (Category 4)

Acute toxicity, Dermal (Category 5)

Skin irritation (Category 3 )

Serious eye damage (Category 1 )

Specific target organ toxicity - single exposure (Category 3 )

GHS Label elements, including precautionary statements

Pictogram

Signal word

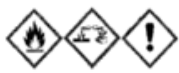

Hazard statement(s)

H225

$\mathrm{H} 302$

H313

H316

H318

$\mathrm{H} 335+\mathrm{H} 336$

Danger

Highly flammable liquid and vapour.

Harmful if swallowed.

May be harmful in contact with skin.

Causes mild skin irntation.

Causes serious eye damage.

May cause respiratory irritation, and drowsiness or dizziness. 
Precautionary statement(s)

P210

P261

$P 305+P 351+P 338$

HMIS Classification

Health hazard:

Chronic Health Hazard:

Flammability:

Physical hazards:

NFPA Rating

Health hazard:

Fire:

Reactivity Hazard:

Potential Health Effects

Inhalation

Skin

Eyes

Ingestion
Keep away from heat/sparks/open flames/hot surfaces. - No smoking

Avoid breathing dust/ fume/ gas/ mist/ vapours/ spray.

Wear protective gloves/ eye protection/ face protection.

IF IN EYES: Rinse cautiously with water for several minutes. Remove contact lenses, if present and easy to do. Continue rinsing.

\section{COMPOSITION/INFORMATION ON INGREDIENTS}

\begin{tabular}{|c|c|c|c|}
\hline Synonyms & THF & & \\
\hline $\begin{array}{l}\text { Formula } \\
\text { Molecular Weight }\end{array}$ & $\begin{array}{l}: \quad \mathrm{C} 4 \mathrm{H} 8 \mathrm{O} \\
: \quad 72.11 \mathrm{~g} / \mathrm{mol}\end{array}$ & & \\
\hline CAS-No. & EC-No. & Index-No. & Concentration \\
\hline \multicolumn{4}{|l|}{ Tetrahydrofuran } \\
\hline $109-99-9$ & $203-726-8$ & $603-025-00-0$ & - \\
\hline
\end{tabular}

\section{FIRST AID MEASURES}

General advice

Consult a physician. Show this safety data sheet to the doctor in attendance.Move out of dangerous area.

If inhaled

If breathed in, move person into fresh air. If not breathing, give artificial respiration. Consult a physician.

In case of skin contact

Wash off with soap and plenty of water. Consult a physician.

In case of eye contact

Rinse thoroughly with plenty of water for at least 15 minutes and consult a physician.

If swallowed

Do NOT induce vomiting. Never give anything by mouth to an unconscious person. Rinse mouth with water. Consult a

physician.

\section{FIRE-FIGHTING MEASURES}

Conditions of flammability

Flammable in the presence of a source of ignition when the temperature is above the flash point. Keep away from heat/sparks/open flame/hot surface. No smoking.

Suitable extinguishing media

Use water spray, alcohol-resistant foam, dry chemical or carbon dioxide.

Special protective equipment for fire-fighters

Wear self contained breathing apparatus for fire fighting if necessary.

Sigma-Aldrich - 401757 
Hazardous combustion products

Hazardous decomposition products formed under fire conditions. - Carbon oxides

Further information

Use water spray to cool unopened containers.

\section{ACCIDENTAL RELEASE MEASURES}

Personal precautions

Use personal protective equipment. Avoid breathing vapors, mist or gas. Ensure adequate ventilation. Remove all sources of ignition. Evacuate personnel to safe areas. Beware of vapours accumulating to form explosive

concentrations. Vapours can accumulate in low areas.

Environmental precautions

Prevent further leakage or spillage if safe to do so. Do not let product enter drains.

Methods and materials for containment and cleaning up

Contain spillage, and then collect with an electrically protected vacuum cleaner or by wet-brushing and place in container for disposal according to local regulations (see section 13).

7. HANDLING AND STORAGE

Precautions for safe handling

Avoid contact with skin and eyes. Avoid inhalation of vapour or mist.

Use explosion-proof equipment. Keep away from sources of ignition - No smoking. Take measures to prevent the build up of electrostatic charge.

Conditions for safe storage

Keep container tightly closed in a dry and well-ventilated place. Containers which are opened must be carefully resealed and kept upright to prevent leakage.

\section{EXPOSURE CONTROLS/PERSONAL PROTECTION}

Components with workplace control parameters

\begin{tabular}{|c|c|c|c|c|}
\hline Components & CAS-No. & Value & $\begin{array}{l}\text { Control } \\
\text { parameters }\end{array}$ & Basis \\
\hline Tetrahydrofuran & $109-99-9$ & TWA & $50 \mathrm{ppm}$ & USA. ACGIH Threshold Limit Values (TLV) \\
\hline \multirow[t]{2}{*}{ Remarks } & \multicolumn{4}{|c|}{$\begin{array}{l}\text { Central Nervous System impairment Upper Respiratory Tract irritation Kidney damage Confirmed } \\
\text { animal carcinogen with unknown relevance to humans: The agent is carcinogenic in experimental } \\
\text { animals at a relatively high dose, by route(s) of administration, at site(s), of histologic type(s), or by } \\
\text { mechanism(s) that may not be relevant to worker exposure. Available epidemiologic studies do not } \\
\text { confirm an increased risk of cancer in exposed humans. Available evidence does not suggest that } \\
\text { the agent is likely to cause cancer in humans except under uncommon or unlikely routes or levels of } \\
\text { exposure. Danger of cutaneous absorption }\end{array}$} \\
\hline & & STEL & $100 \mathrm{ppm}$ & USA. ACGIH Threshold Limit Values (TLV) \\
\hline . & \multicolumn{4}{|c|}{$\begin{array}{l}\text { Central Nervous System impairment Upper Respiratory Tract irritation Kidney damage Confirmed } \\
\text { animal carcinogen with unknown relevance to humans: The agent is carcinogenic in experimental } \\
\text { animals at a relatively high dose, by route(s) of administration, at site(s), of histologic type(s), or by } \\
\text { mechanism(s) that may not be relevant to worker exposure. Available epidemiologic studies do not } \\
\text { confirm an increased risk of cancer in exposed humans. Available evidence does not suggest that } \\
\text { the agent is likely to cause cancer in humans except under uncommon or unlikely routes or levels of } \\
\text { exposure. Danger of cutaneous absorption }\end{array}$} \\
\hline & & TWA & $\begin{array}{l}200 \mathrm{ppm} \\
590 \mathrm{mg} / \mathrm{m} 3\end{array}$ & $\begin{array}{l}\text { USA. OSHA - TABLE Z-1 Limits for Air Contaminants - } \\
1910.1000\end{array}$ \\
\hline & & STEL & $\begin{array}{l}250 \mathrm{ppm} \\
735 \mathrm{mg} / \mathrm{m} 3\end{array}$ & $\begin{array}{l}\text { USA. OSHA - TABLE Z-1 Limits for Air Contaminants - } \\
1910.1000\end{array}$ \\
\hline & & TWA & $\begin{array}{l}200 \mathrm{ppm} \\
590 \mathrm{mg} / \mathrm{m} 3\end{array}$ & $\begin{array}{l}\text { USA. Occupational Exposure Limits (OSHA) - Table Z-1 } \\
\text { Limits for Air Contaminants }\end{array}$ \\
\hline
\end{tabular}


Hazardous combustion products

Hazardous decomposition products formed under fire conditions. - Carbon oxides

Further information

Use water spray to cool unopened containers.

\section{ACCIDENTAL RELEASE MEASURES}

Personal precautions

Use personal protective equipment. Avoid breathing vapors, mist or gas. Ensure adequate ventilation. Remove all sources of ignition. Evacuate personnel to safe areas. Beware of vapours accumulating to form explosive concentrations. Vapours can accumulate in low areas.

Environmental precautions

Prevent further leakage or spillage if safe to do so. Do not let product enter drains.

Methods and materials for containment and cleaning up

Contain spillage, and then collect with an electricaliy protected vacuum cleaner or by wet-brushing and place in container for disposal according to local regulations (see section 13).

\section{HANDLING AND STORAGE}

Precautions for safe handling

Avoid contact with skin and eyes. Avoid inhalation of vapour or mist.

Use explosion-proof equipment. Keep away from sources of ignition - No smoking. Take measures to prevent the build up of electrostatic charge.

Conditions for safe storage

Keep container tightly closed in a dry and well-ventilated place. Containers which are opened must be carefully resealed and kept upright to prevent leakage.

\section{EXPOSURE CONTROLS/PERSONAL PROTECTION}

Components with workplace control parameters

\begin{tabular}{|c|c|c|c|c|}
\hline Components & CAS-No. & Value & $\begin{array}{l}\text { Control } \\
\text { parameters }\end{array}$ & Basis \\
\hline Tetrahydrofuran & $109-99-9$ & TWA & $50 \mathrm{ppm}$ & USA. ACGIH Threshold Limit Values (TLV) \\
\hline Remarks & \multicolumn{4}{|c|}{$\begin{array}{l}\text { Central Nervous System impairment Upper Respiratory Tract irritation Kidney damage Confirmed } \\
\text { animal carcinogen with unknown relevance to humans: The agent is carcinogenic in experimental } \\
\text { animals at a relatively high dose, by route(s) of administration, at site(s), of histologic type(s), or by } \\
\text { mechanism(s) that may not be relevant to worker exposure. Available epidemiologic studies do not } \\
\text { confirm an increased risk of cancer in exposed humans. Available evidence does not suggest that } \\
\text { the agent is likely to cause cancer in humans except under uncommon or unlikely routes or levels of } \\
\text { exposure. Danger of cutaneous absorption }\end{array}$} \\
\hline & & STEL & $100 \mathrm{ppm}$ & USA. ACGIH Threshold Limit Values (TLV) \\
\hline & \multicolumn{4}{|c|}{$\begin{array}{l}\text { Central Nervous System impairment Upper Respiratory Tract irritation Kidney damage Confirmed } \\
\text { animal carcinogen with unknown relevance to humans: The agent is carcinogenic in experimental } \\
\text { animals at a relatively high dose, by route(s) of administration, at site(s), of histologic type(s), or by } \\
\text { mechanism(s) that may not be relevant to worker exposure. Available epidemiologic studies do not } \\
\text { confirm an increased risk of cancer in exposed humans. Available evidence does not suggest that } \\
\text { the agent is likely to cause cancer in humans except under uncommon or unlikely routes or levels of } \\
\text { exposure. Danger of cutaneous absorption }\end{array}$} \\
\hline & & TWA & $\begin{array}{l}200 \mathrm{ppm} \\
590 \mathrm{mg} / \mathrm{m} 3\end{array}$ & $\begin{array}{l}\text { USA. OSHA - TABLE Z-1 Limits for Air Contaminants - } \\
1910.1000\end{array}$ \\
\hline & & STEL & $\begin{array}{l}250 \mathrm{ppm} \\
735 \mathrm{mg} / \mathrm{m} 3\end{array}$ & $\begin{array}{l}\text { USA. OSHA - TABLE Z-1 Limits for Air Contaminants - } \\
1910.1000\end{array}$ \\
\hline & & TWA & $\begin{array}{l}200 \mathrm{ppm} \\
590 \mathrm{mg} / \mathrm{m} 3\end{array}$ & $\begin{array}{l}\text { USA. Occupational Exposure Limits (OSHA) - Table Z-1 } \\
\text { Limits for Air Contaminants }\end{array}$ \\
\hline
\end{tabular}




\begin{tabular}{|c|c|c|c|c|}
\hline \multicolumn{5}{|c|}{ The value in $\mathrm{mg} / \mathrm{m} 3$ is approximate. } \\
\hline & & TWA & $\begin{array}{l}200 \mathrm{ppm} \\
590 \mathrm{mg} / \mathrm{m} 3\end{array}$ & USA. NIOSH Recommended Exposure Limits \\
\hline & & ST & $\begin{array}{l}250 \mathrm{ppm} \\
735 \mathrm{mg} / \mathrm{m} 3\end{array}$ & USA. NIOSH Recommended Exposure Limits \\
\hline
\end{tabular}

Personal protective equipment

Respiratory protection

Where risk assessment shows air-purifying respirators are appropriate use a full-face respirator with multi-purpose combination (US) or type AXBEK (EN 14387) respirator cartridges as a backup to engineering controls. If the respirator is the sole means of protection, use a full-face supplied air respirator. Use respirators and components tested and approved under appropriate government standards such as NIOSH (US) or CEN (EU).

Hand protection

Handle with gloves. Gloves must be inspected prior to use. Use proper glove removal technique (without touching glove's outer surface) to avoid skin contact with this product. Dispose of contaminated gloves after use in accordance with applicable laws and good laboratory practices. Wash and dry hands.

Eye protection

Tightly fitting safety goggles. Faceshield (8-inch minimum). Use equipment for eye protection tested and approved under appropriate government standards such as NIOSH (US) or EN 166(EU).

Skin and body protection

Complete suit protecting against chemicals, Flame retardant antistatic protective clothing. The type of protective equipment must be selected according to the concentration and amount of the dangerous substance at the specific workplace.

Hygiene measures

Handle in accordance with good industrial hygiene and safety practice. Wash hands before breaks and at the end of workday.

9. PHYSICAL AND CHEMICAL PROPERTIES

Appearance

Form

liquid, clear

Colour

colourless

Safety data

$\mathrm{pH}$

Melting

point/freezing point

Boiling point

no data available

$-108.0^{\circ} \mathrm{C}\left(-162.4^{\circ} \mathrm{F}\right)$

Flash point

$65.0-67.0^{\circ} \mathrm{C}\left(149.0-152.6^{\circ} \mathrm{F}\right)$

Ignition temperature

Autoignition

temperature

Lower explosion limit

$-17.0^{\circ} \mathrm{C}\left(1.4^{\circ} \mathrm{F}\right)$ - closed cup

Upper explosion limit

Vapour pressure

$321^{\circ} \mathrm{C}\left(610^{\circ} \mathrm{F}\right)$

$321.0^{\circ} \mathrm{C}\left(609.8^{\circ} \mathrm{F}\right)$

$1.8 \%(\mathrm{~V})$

$11.8 \%(\mathrm{~V})$

$152.0 \mathrm{hPa}(114.0 \mathrm{mmHg})$ at $15.0^{\circ} \mathrm{C}\left(59.0^{\circ} \mathrm{F}\right)$

$190.7 \mathrm{hPa}(143.0 \mathrm{mmHg})$ at $20.0^{\circ} \mathrm{C}\left(68.0^{\circ} \mathrm{F}\right)$

$213.3 \mathrm{hPa}(160.0 \mathrm{mmHg})$ at $25.0^{\circ} \mathrm{C}\left(77.0^{\circ} \mathrm{F}\right)$

Density

$373.3 \mathrm{hPa}(280.0 \mathrm{mmHg})$ at $38.0^{\circ} \mathrm{C}\left(100.4^{\circ} \mathrm{F}\right)$

Water solubility

$\mathrm{g} / \mathrm{cm} 3$

Partition coefficient:

soluble

n-octanol/water

Sigma-Aldrich - 401757 


\begin{tabular}{|c|c|c|c|c|}
\hline & \multicolumn{4}{|c|}{ The value in $\mathrm{mg} / \mathrm{m} 3$ is approximate. } \\
\hline & & TWA & $\begin{array}{l}200 \mathrm{ppm} \\
590 \mathrm{mg} / \mathrm{m} 3\end{array}$ & USA. NIOSH Recommended Exposure Limits \\
\hline & & ST & $\begin{array}{l}250 \mathrm{ppm} \\
735 \mathrm{mg} / \mathrm{m} 3\end{array}$ & USA. NIOSH Recommended Exposure Limits \\
\hline
\end{tabular}

Personal protective equipment

Respiratory protection

Where risk assessment shows air-purifying respirators are appropriate use a full-face respirator with multi-purpose combination (US) or type AXBEK (EN 14387) respirator cartridges as a backup to engineering controls. If the respirator is the sole means of protection, use a full-face supplied air respirator. Use respirators and components tested and approved under appropriate government standards such as NIOSH (US) or CEN (EU).

Hand protection

Handle with gloves. Gloves must be inspected prior to use. Use proper glove removal technique (without touching glove's outer surface) to avoid skin contact with this product. Dispose of contaminated gloves after use in accordance with applicable laws and good laboratory practices. Wash and dry hands.

Eye protection

Tightly fitting safety goggles. Faceshield (8-inch minimum). Use equipment for eye protection tested and approved under appropriate government standards such as NIOSH (US) or EN 166(EU).

Skin and body protection

Complete suit protecting against chemicals, Flame retardant antistatic protective clothing. The type of protective equipment must be selected according to the concentration and amount of the dangerous substance at the specific workplace.

Hygiene measures

Handle in accordance with good industrial hygiene and safety practice. Wash hands before breaks and at the end of workday.

9. PHYSICAL AND CHEMICAL PROPERTIES

Appearance

Form

liquid, clear

Colour

colourless

Safety data

$\mathrm{pH}$

Melting

no data available

point/freezing point

Boiling point

$-108.0^{\circ} \mathrm{C}\left(-162.4^{\circ} \mathrm{F}\right)$

Flash point

$65.0-67.0^{\circ} \mathrm{C}\left(149.0-152.6^{\circ} \mathrm{F}\right)$

Ignition temperature

Autoignition

temperature

Lower explosion limit

$-17.0^{\circ} \mathrm{C}\left(1.4^{\circ} \mathrm{F}\right)$ - closed cup

Upper explosion limit

Vapour pressure

$321^{\circ} \mathrm{C}\left(610^{\circ} \mathrm{F}\right)$

$321.0^{\circ} \mathrm{C}\left(609.8^{\circ} \mathrm{F}\right)$

$1.8 \%(\mathrm{~V})$

$11.8 \%(\mathrm{~V})$

$152.0 \mathrm{hPa}(114.0 \mathrm{mmHg})$ at $15.0^{\circ} \mathrm{C}\left(59.0^{\circ} \mathrm{F}\right)$

$190.7 \mathrm{hPa}(143.0 \mathrm{mmHg})$ at $20.0^{\circ} \mathrm{C}\left(68.0^{\circ} \mathrm{F}\right)$

$213.3 \mathrm{hPa}(160.0 \mathrm{mmHg})$ at $25.0^{\circ} \mathrm{C}\left(77.0^{\circ} \mathrm{F}\right)$

Density

$373.3 \mathrm{hPa}(280.0 \mathrm{mmHg})$ at $38.0^{\circ} \mathrm{C}\left(100.4^{\circ} \mathrm{F}\right)$

Water solubility

Partition coefficient:

soluble

n-octanol/water

Sigma-Aldrich - 401757 


$\begin{array}{ll}\text { Viscosity, kinematic } & 0.512 \mathrm{~mm} 2 / \mathrm{s} \text { at } 25^{\circ} \mathrm{C}\left(77^{\circ} \mathrm{F}\right) \\ & 0.403 \mathrm{~mm} 2 / \mathrm{s} \text { at } 50^{\circ} \mathrm{C}\left(122^{\circ} \mathrm{F}\right) \\ \begin{array}{l}\text { Relative vapour } \\ \text { density }\end{array} & \text { no data available } \\ \text { Odour } & \text { no data available } \\ \text { Odour Threshold } & \text { no data available } \\ \text { Evaporation rate } & \text { no data available }\end{array}$

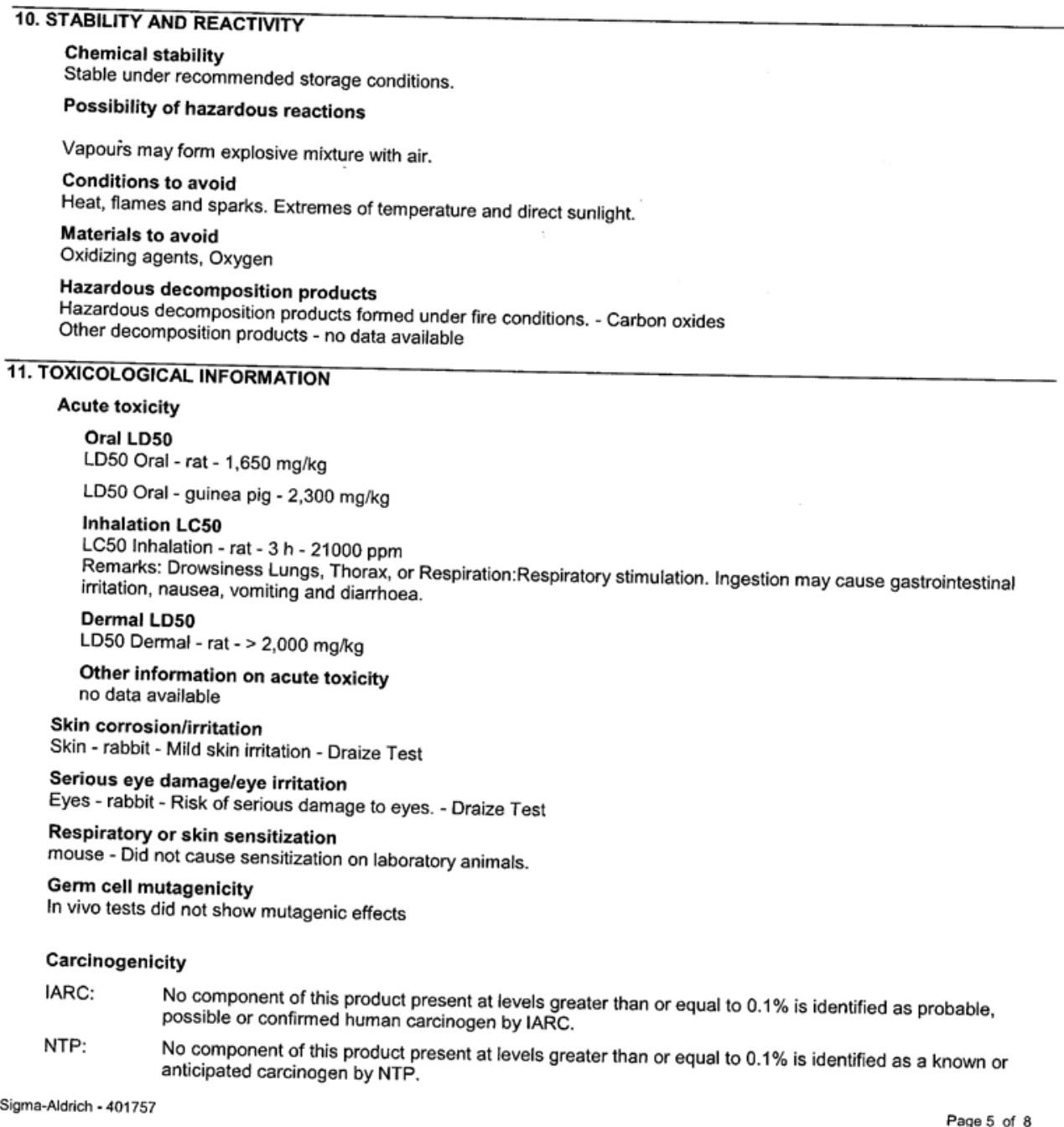


OSHA: $\quad$ No component of this product present at levels greater than or equal to $0.1 \%$ is identified as a carcinogen or potential carcinogen by OSHA.

Reproductive toxicity

No toxicity to reproduction

Teratogenicity

no data available

Specific target organ toxicity - single exposure (Globally Harmonized System)

Inhalation - May cause respiratory irritation.

May cause drowsiness or dizziness. - Nervous system

Specific target organ toxicity - repeated exposure (Globally Harmonized System)

The substance or mixture is not classified as specific target organ toxicant, repeated exposure.

Aspiration hazard

No aspiration toxicity classification

Potential health effects

Inhalation

May be harmful if inhaled. Causes respiratory tract irritation. Vapours may cause

Ingestion

drowsiness and dizziness.

Harmful if swallowed.

Skin Harmful if absorbed through skin. Causes skin irritation.

Eyes Causes eye irritation.

Signs and Symptoms of Exposure

Central nervous system depression, Cough, chest pain, Difficulty in breathing, Exposure to high airborne concentrations can cause anesthetic effects., To the best of our knowledge, the chemical, physical, and toxicological properties have not been thoroughly investigated.

Synergistic effects

no data available

Additional Information

RTECS: LU5950000

12. ECOLOGICAL. INFORMATION

Toxicity

Toxicity to fish LC50 - Pimephales promelas (fathead minnow) $-2,160 \mathrm{mg} / \mathrm{l}-96 \mathrm{~h}$

Toxicity to algae Growth inhibition NOEC - Algae - 3,700 mg/l - $192 \mathrm{~h}$

Persistence and degradability

Expected to be biodegradable

Bioaccumulative potential

no data available

Mobility in soil

no data available

PBT and vPvB assessment

no data available

Other adverse effects

no data available

13. DISPOSAL CONSIDERATIONS 
Product

Burn in a chemical incinerator equipped with an afterburner and scrubber but exert extra care in igniting as this material is highly flammable. Offer surplus and non-recyclable solutions to a licensed disposal company. Contact a licensed professional waste disposal service to dispose of this material.

Contaminated packaging

Dispose of as unused product.

14. TRANSPORT INFORMATION

DOT (US)

UN number: 2056 Class: 3

Proper shipping name: Tetrahydrofuran

Packing group: II

Proper shipping name: Tetrahydrofur
Reportable Quantity (RQ): 1000 lbs

Marine pollutant: No

Poison Inhalation Hazard: No

IMDG-

UN number: 2056 Class: 3

Proper shipping name: TETRAHYDROFURAN

Marine pollutant: No

IATA

UN number: 2056 Class: 3

Proper shipping name: Tetrahydrofuran

Packing group: II

15. REGULATORY INFORMATION

OSHA Hazards

Flammable liquid, Target Organ Effect, Harmful by ingestion., Irritant, Carcinogen

SARA 302 Components

SARA 302: No chemicals in this material are subject to the reporting requirements of SARA Title III, Section 302.

SARA 313 Components

SARA 313: This material does not contain any chemical components with known CAS numbers that exceed the threshold (De Minimis) reporting levels established by SARA Title III, Section 313.

SARA 311/312 Hazards

Fire Hazard, Acute Health Hazard, Chronic Health Hazard

Massachusetts Right To Know Components

Tetrahydrofuran

$\begin{array}{ll}\text { CAS-No. } & \text { Revision Date } \\ \text { 109-99-9 } & 2007-03-01\end{array}$

Pennsylvania Right To Know Components

Tetrahydrofuran

CAS-No. Revision Date

New Jersey Right To Know Components

Tetrahydrofuran

California Prop. 65 Components

This product does not contain any chemicals known to State of California to cause cancer, birth defects, or any other reproductive harm.

\section{OTHER INFORMATION}

Further information

Copyright 2011 Sigma-Aldrich Co. License granted to make unlimited paper copies for internal use only.

The above information is believed to be correct but does not purport to be all inclusive and shall be used only as a

guide. The information in this document is based on the present state of our knowledge and is applicable to the

product with regard to appropriate safety precautions. It does not represent any guarantee of the properties of the

product. Sigma-Aldrich $\mathrm{Co}$., shall not be held liable for any damage resulting from handling or from contact with the

above product. See reverse side of invoice or packing slip for additional terms and conditions of sale. 


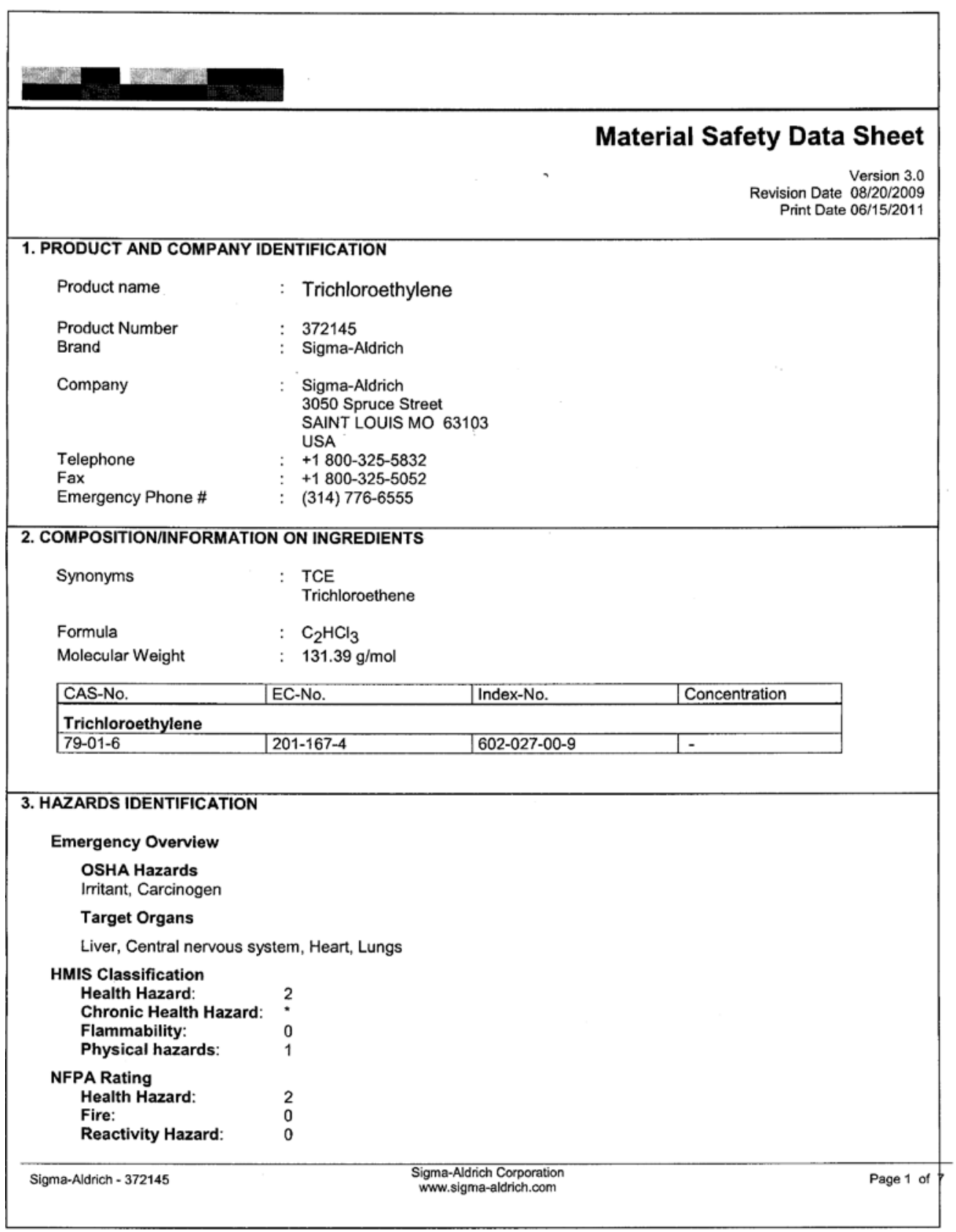




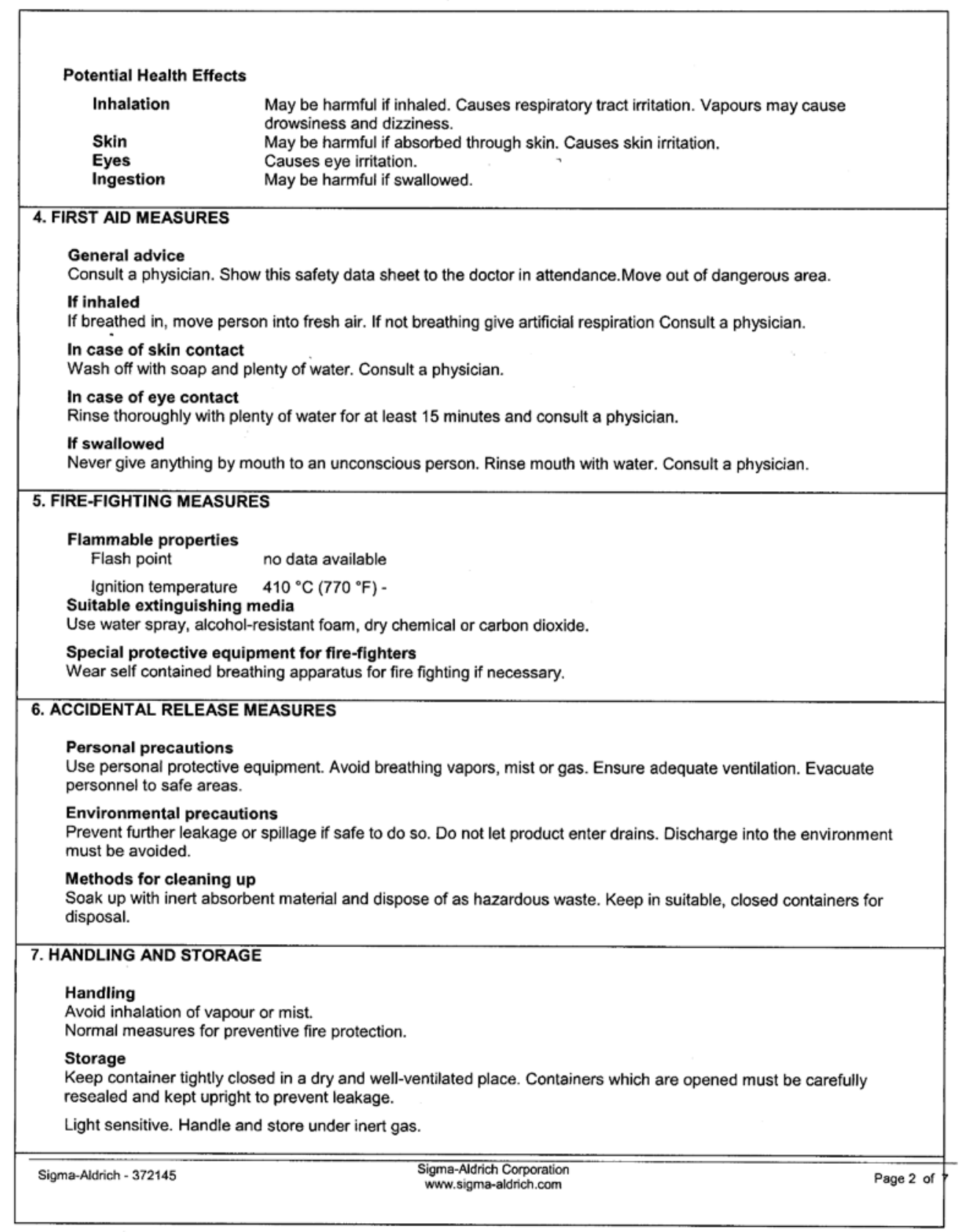


8. EXPOSURE CONTROLS/PERSONAL PROTECTION

Components with workplace control parameters

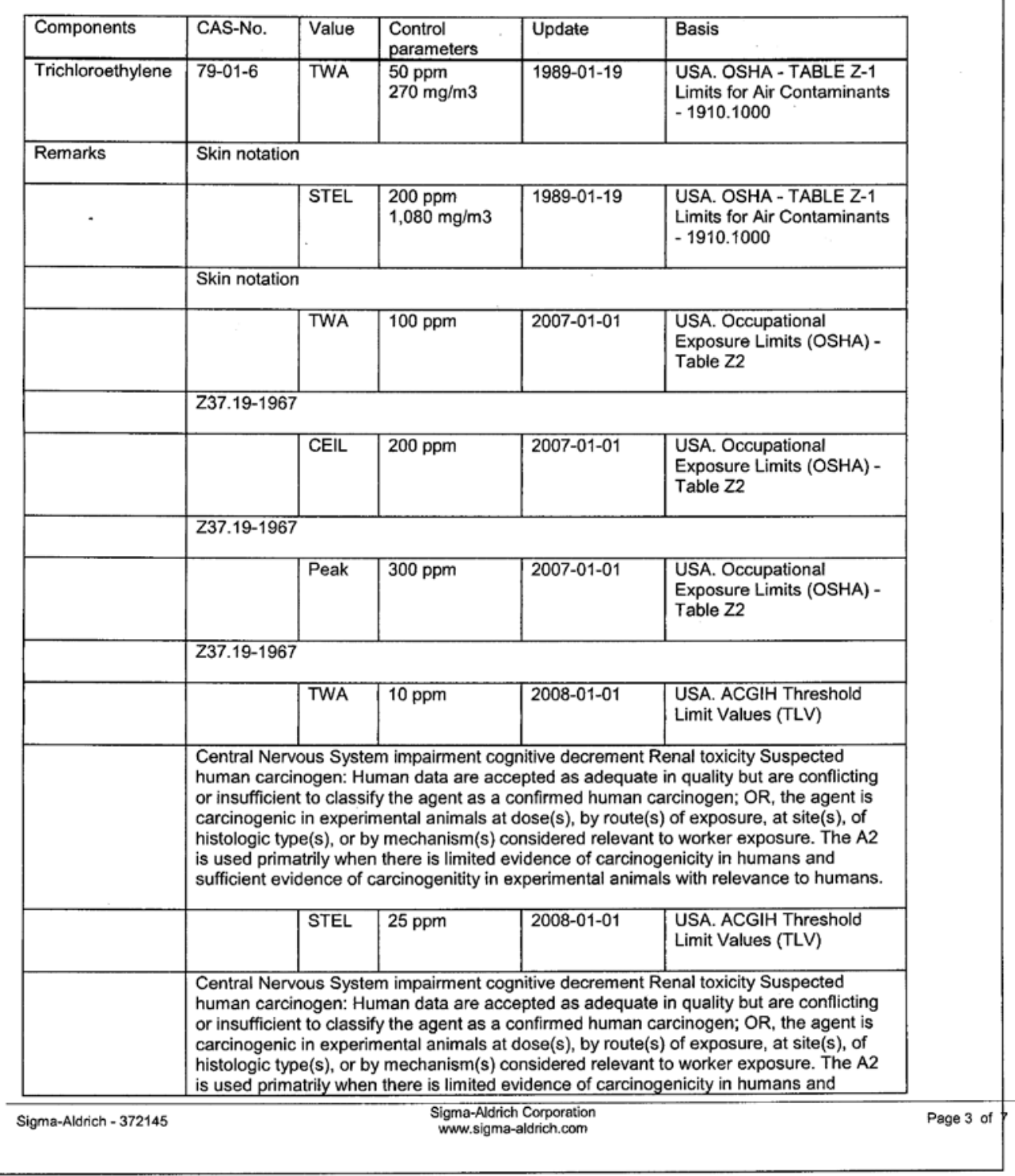


sufficient evidence of carcinogenitity in experimental animals with relevance to humans.

Personal protective equipment

Respiratory protection

Where risk assessment shows air-purifying respirators are appropriate use a full-face respirator with multi-

purpose combination (US) or type ABEK (EN 14387) respirator cartridges as a backup to engineering controls. If

the respirator is the sole means of protection, use a full-face supplied air respirator. Use respirators and

components tested and approved under appropriate government standards such as NIOSH (US) or CEN (EU).

Hand protection

Handle with gloves.

Eye protection

Safety glasses with side-shields conforming to EN166

Skin and body protection

Choose body protection according to the amount and concentration of the dangerous substance at the work

place.

Hygiene measures

Avoid contact with skin, eyes and clothing. Wash hands before breaks and immediately after handling the product.

\section{PHYSICAL AND CHEMICAL PROPERTIES}

\section{Appearance}

Form

liquid, clear

Colour

colourless

Safety data

$\mathrm{pH}$

Melting point

no data available

Boiling point

$-84.8^{\circ} \mathrm{C}\left(-120.6^{\circ} \mathrm{F}\right)-$ lit.

Flash point

$86.7^{\circ} \mathrm{C}\left(188.1^{\circ} \mathrm{F}\right)-$ lit.

Ignition temperature $410^{\circ} \mathrm{C}\left(770^{\circ} \mathrm{F}\right)$ -

Lower explosion limit $8 \%(\mathrm{~V})$

Upper explosion limit $10.5 \%(\mathrm{~V})$

Vapour pressure $\quad 81.3 \mathrm{hPa}(61.0 \mathrm{mmHg})$ at $20.0^{\circ} \mathrm{C}\left(68.0^{\circ} \mathrm{F}\right)$

Density

$1.463 \mathrm{~g} / \mathrm{mL}$ at $25^{\circ} \mathrm{C}\left(77^{\circ} \mathrm{F}\right)$

Water solubility

Partition coefficient:

n-octanol/water

no data available

log Pow: 2.29

10. STABILITY AND REACTIVITY

Storage stability

Stable under recommended storage conditions.

Materials to avoid

Oxidizing agents, Strong bases, Magnesium

Sigma-Aldrich - 372145 
Hazardous decomposition products

Hazardous decomposition products formed under fire conditions. - Carbon oxides, Hydrogen chloride gas

Hazardous decomposition products formed under fire conditions. - Nature of decomposition products not known.

\section{TOXICOLOGICAL INFORMATION}

Acute toxicity

LD50 Oral - rat - 4,920 mg/kg

LC50 Inhalation - mouse - $4 \mathrm{~h}-8450 \mathrm{ppm}$

LD50 Dermal - rabbit - > 20,000 mg/kg

Irritation and corrosion

Skin - rabbit - Severe skin irritation - $24 \mathrm{~h}$

Eyes - rabbit - Eye irritation - $24 \mathrm{~h}$

Sensitisation

no data available

Chronic exposure

This product is or contains a component that has been reported to be probably carcinogenic based on its IARC, OSHA, ACGIH, NTP, or EPA classification.

IARC: $\quad 2 A-$ Group 2A: Probably carcinogenic to humans (Trichloroethylene)

NTP: $\quad$ Reasonably anticipated to be a human carcinogen (Trichloroethylene)

OSHA: $\quad$ No component of this product present at levels greater than or equal to $0.1 \%$ is identified as a carcinogen or potential carcinogen by OSHA.

Laboratory experiments have shown mutagenic effects.

\section{Signs and Symptoms of Exposure}

burning sensation, Cough, wheezing, laryngitis, Shortness of breath, Headache, Nausea, Vomiting, Exposure to and/or consumption of alcohol may increase toxic effects., Gastrointestinal disturbance, Kidney injury may occur. narcosis

Potential Health Effects

Inhalation May be harmful if inhaled. Causes respiratory tract irritation. Vapours may cause

drowsiness and dizziness.

Skin May be harmful if absorbed through skin. Causes skin irritation.

Eyes Causes eye irritation.

Ingestion May be harmful if swallowed.

Target Organs Liver, Central nervous system, Heart, Lungs,

Additional Information

RTECS: KX4550000

12. ECOLOGICAL INFORMATION

Elimination information (persistence and degradability)

Ecotoxicity effects

Toxicity to fish $\quad$ LC50 - Pimephales promelas (fathead minnow) $-41.00 \mathrm{mg} / \mathrm{l}-96 \mathrm{~h}$

Sigma-Aldrich - 372145 


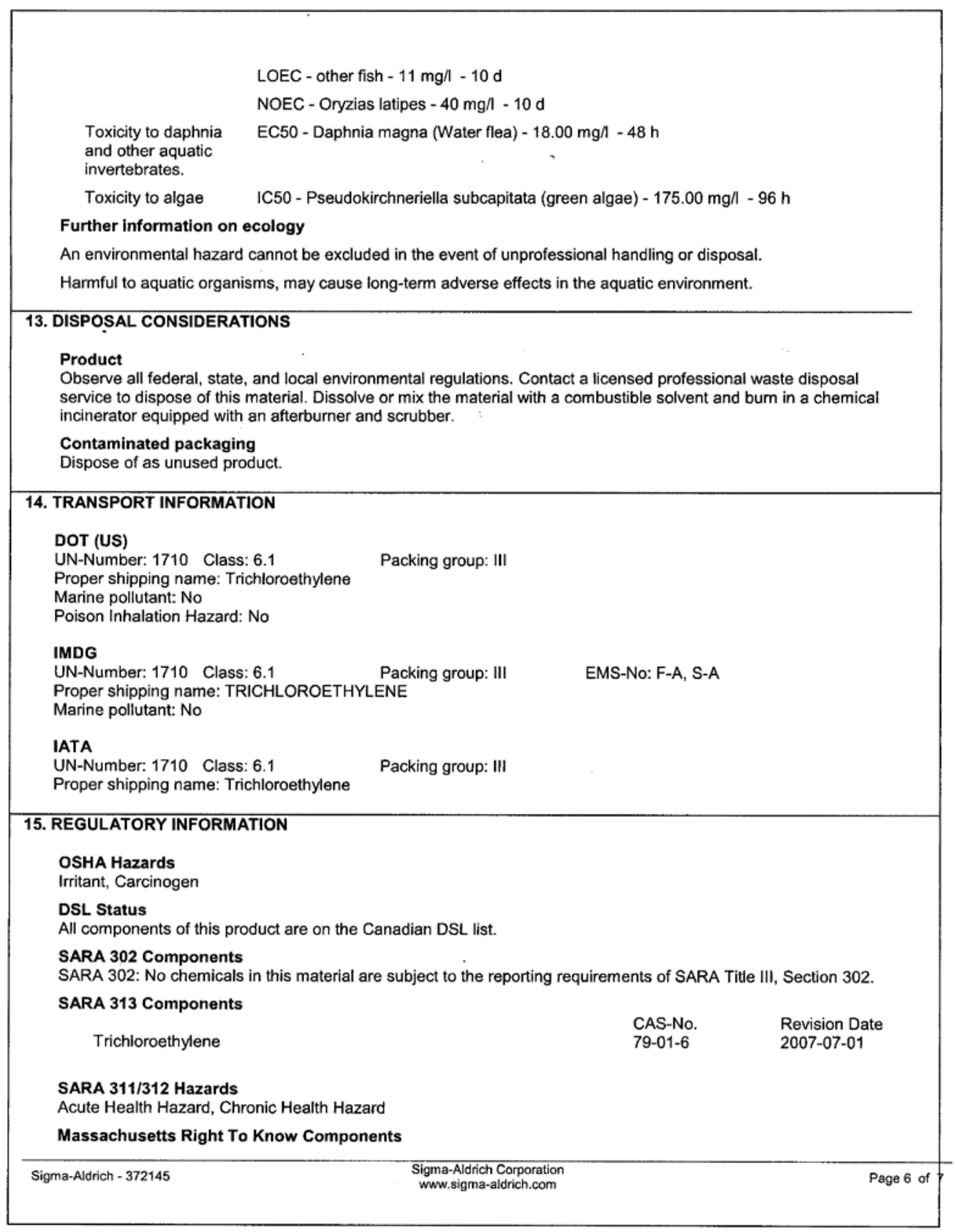




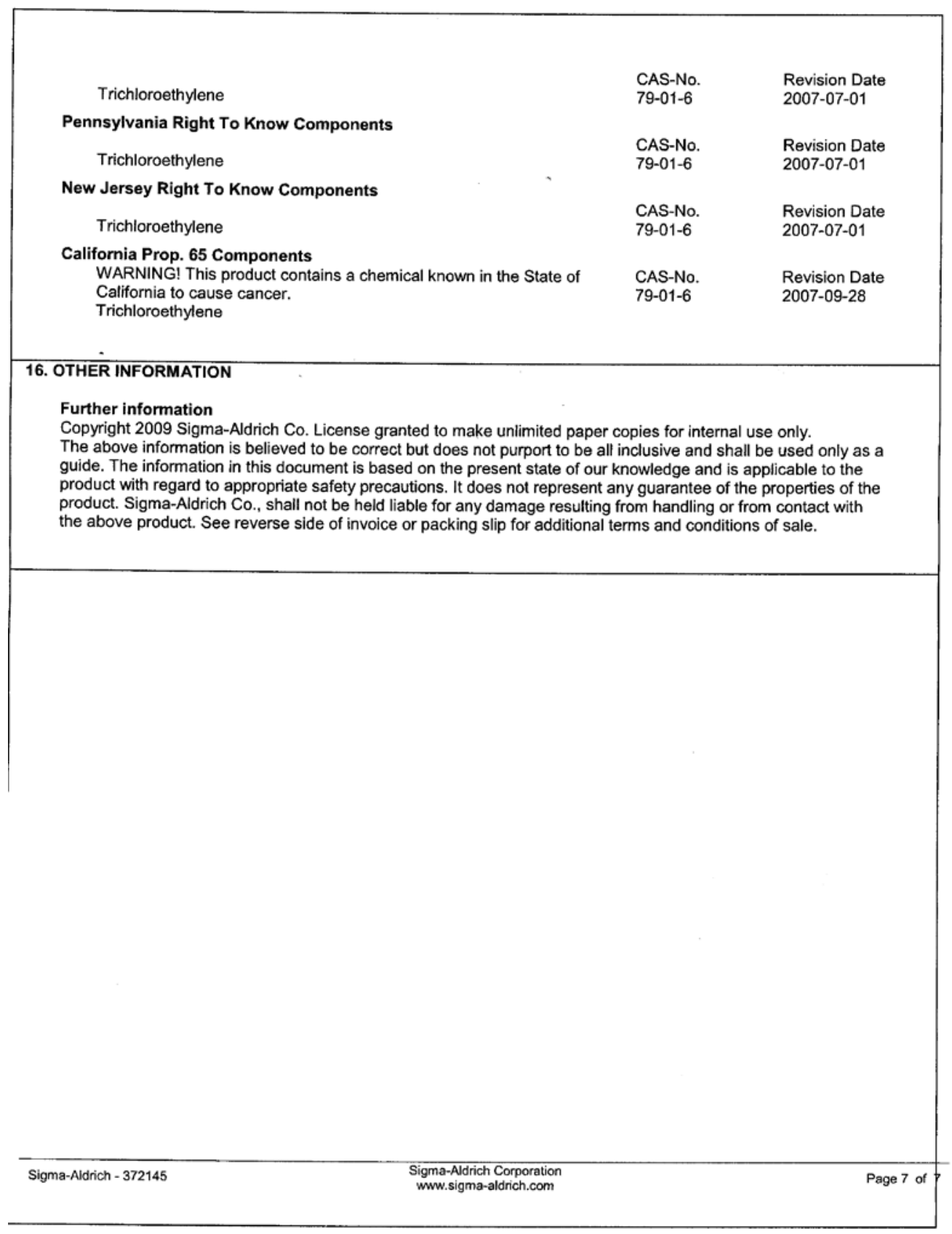




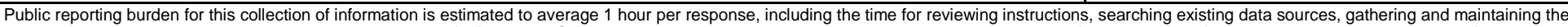

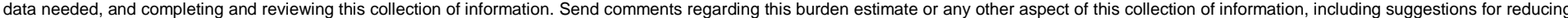

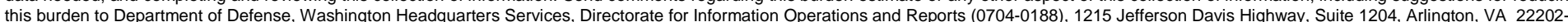

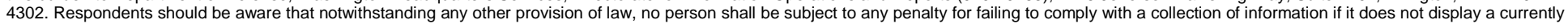
valid OMB control number. PLEASE DO NOT RETURN YOUR FORM TO THE ABOVE ADDRESS.
1. REPORT DATE (DD-MM-YYYY)
06-02-2014

4. TITLE AND SUBTITLE

Lipophilic Super-Absorbent Swelling Gels as Cleaners for Use on Weapons Systems and Platforms

\section{AUTHOR(S)}

Veera M. Boddu, Sophie Minori Uchimiya, Masahiko Ohta, Kazuki Sada, Christopher Myers, Wayne

Ziegler, and Thomas Torres

\section{PERFORMING ORGANIZATION NAME(S) AND ADDRESS(ES)}

U.S. Army Engineer Research and Development Center (ERDC)

Construction Engineering Research Laboratory (CERL)

PO Box 9005,

Champaign, IL 61826-9005

\section{SPONSORING I MONITORING AGENCY NAME(S) AND ADDRESS(ES)}

Strategic Environmental Research and Developemnt Program (SERDP)

901 North Stuart St., Suite 303

Arlington, VA 22203

\section{DISTRIBUTION / AVAILABILITY STATEMENT}

Approved for public release; distribution is unlimited.

\section{DATES COVERED (From - To)}

5a. CONTRACT NUMBER

\section{5b. GRANT NUMBER}

\section{5c. PROGRAM ELEMENT}

\section{5d. PROJECT NUMBER}

SERDP

5e. TASK NUMBER

5f. WORK UNIT NUMBER

WP-1761

8. PERFORMING ORGANIZATION REPORT NUMBER

ERDC/ CERL TR-14-2

\section{SUPPLEMENTARY NOTES}

\section{ABSTRACT}

Increasingly stringent environmental regulations on volatile organic compounds (VOCs) and hazardous air pollutants (HAPs) demand the development of disruptive technologies for cleaning weapons systems and platforms. Currently employed techniques suffer from safety and efficiency shortcomings. This work proposed to use novel lipophilic super-absorbent swelling gels as a disruptive solid state cleaning technology to overcome limitations of currently employed cleaning techniques. Results were compared with a commercially available alkylstyrene copolymer (imbiber beads). The cleaning ability of the gels was compared with the standard solvent cleaner trichloroethylene (TCE) following American Society for Testing and Materials (ASTM) G122-96(2008) test methods and MIL-PRF680B procedure with MIL-PRF-10924 test grease. Polymer gel cleaners exhibited analogues extent and rate of cleaning as the TCE. The work concludes that the recyclable superabsorbent polymer cleaners developed in this research will allow drastic reduction in the use of VOC containing solvents and HAP release.

\section{SUBJECT TERMS}

lipophylic polyelectrolyte gel, alkylsterene copolymer, Stearylacrylate-co-ethylene glycol dimethacryale neutral gel (NG-18), octadecylacrylate-co-ethylene glycol dimethacrylate polyelectrolyte gel (EG-18), surface \& gel cleaning, VOCs \& HAPs reduction

\section{SECURITY CLASSIFICATION OF:}

\begin{tabular}{|l}
\hline a. REPORT \\
Unclassified
\end{tabular}

\section{c. THIS PAGE \\ Unclassified}

\begin{abstract}
17. LIMITATION OF ABSTRACT
\end{abstract}

Unclassified
18. NUMBER OF PAGES

$\begin{array}{ll}\text { SAR } & 72\end{array}$

19a. NAME OF RESPONSIBLE PERSON

\section{9b. TELEPHONE NUMBER} (include area code) 